\title{
VLSI Implementation of a Montgomery Modular
}

\section{Multiplier}

\author{
By \\ Xin Wang \\ A thesis submitted to the Faculty of Graduate Studies and Research \\ in partial fulfillment of the requirements for the degree of \\ Master of Applied Science \\ Ottawa-Carleton Institute for Electrical and Computer Engineering \\ Department of Electronics, Carleton University \\ Ottawa, Ontario, Canada
}

August 2006

(C)2006 Xin Wang 


$\begin{array}{ll}\begin{array}{l}\text { Library and } \\ \text { Archives Canada }\end{array} & \begin{array}{l}\text { Bibliothèque et } \\ \text { Archives Canada }\end{array} \\ \begin{array}{l}\text { Published Heritage } \\ \text { Branch }\end{array} & \begin{array}{l}\text { Direction du } \\ \text { Patrimoine de l'édition }\end{array} \\ \begin{array}{l}\text { 395 Wellington Street } \\ \text { Ottawa ON K1A 0N4 } \\ \text { Canada }\end{array} & \begin{array}{l}\text { 395, rue Wellington } \\ \text { Ottawa ON K1A ON4 } \\ \text { Canada }\end{array}\end{array}$

Your file Votre référence ISBN: 978-0-494-18334-2 Our file Notre référence ISBN: 978-0-494-18334-2

NOTICE:

The author has granted a nonexclusive license allowing Library and Archives Canada to reproduce, publish, archive, preserve, conserve, communicate to the public by telecommunication or on the Internet, loan, distribute and sell theses worldwide, for commercial or noncommercial purposes, in microform, paper, electronic and/or any other formats.

The author retains copyright ownership and moral rights in this thesis. Neither the thesis nor substantial extracts from it may be printed or otherwise reproduced without the author's permission.
AVIS:

L'auteur a accordé une licence non exclusive permettant à la Bibliothèque et Archives Canada de reproduire, publier, archiver, sauvegarder, conserver, transmettre au public par télécommunication ou par l'Internet, prêter, distribuer et vendre des thèses partout dans le monde, à des fins commerciales ou autres, sur support microforme, papier, électronique et/ou autres formats.

L'auteur conserve la propriété du droit d'auteur et des droits moraux qui protège cette thèse. $\mathrm{Ni}$ la thèse ni des extraits substantiels de celle-ci ne doivent être imprimés ou autrement reproduits sans son autorisation.
In compliance with the Canadian

Privacy Act some supporting forms may have been removed from this thesis.

While these forms may be included in the document page count, their removal does not represent any loss of content from the thesis.
Conformément à la loi canadienne sur la protection de la vie privée, quelques formulaires secondaires ont été enlevés de cette thèse.

Bien que ces formulaires aient inclus dans la pagination, il n'y aura aucun contenu manquant.

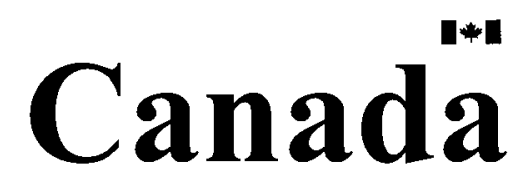




\section{Abstract}

Public-key cryptosystems can provide important security services such as authentication, data integrity and non-repudiation which are not possible with secret-key systems, but they present a much higher computational load and so they are much slower than the latter. An examination of the algorithms used reveals that the processing 'hotspot' in most of the popular systems, such as RSA, ElGamal, Diffie-Hellman and DSA, is the modular multiplication of very large integers -1024 bits or more.

Performance improvement of these systems is therefore equivalent to performance improvement of the modular multiplication operation (in the hardware domain). Relative to the published information, a significant step forward in this regard is described in this thesis.

Architectural developments described herein result in substantial reductions of both the number of clocks per modular multiply and of circuit complexity. Post-layout simulation of a $0.18 \mu \mathrm{m}$ CMOS realization indicates that the new device can operate at 1 $\mathrm{GHz}$ while consuming 1.2 Watts of power (1024-bit word), and requires $1.25 \mathrm{~N}$ clocks to complete an $N$-bit modular multiplication. Radix-4 architecture with $0.75 \mathrm{~N}$ clocks was also proposed. 


\section{Acknowledgements}

First I would like to thank my supervisor, Dr. Tad Kwasniewski, for his advice, patience, guidance, and trust.

A big "Thank You" to my best friends Haitao Mei and Jing Zhang who gave me invaluable help and mental support during the period of my graduate studies.

Thanks must also go out to my colleagues in Department of Electronics at Carleton University, especially Yuntong Song, Yuanzhong Wan for discussions.

This thesis benifitted from the advice and comments of Chenghai Sun and Peter Noel.

Last but not least, I want to thank my wife and my parents, for their continuous love and support. 


\section{Table of Contents}

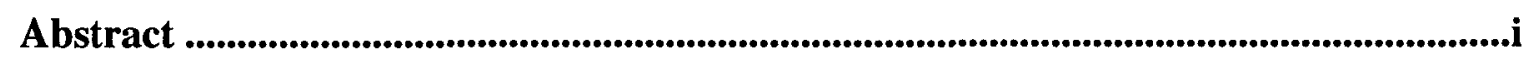

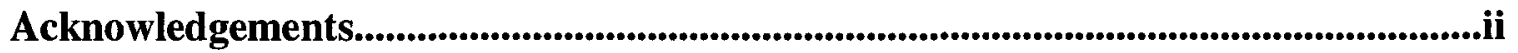

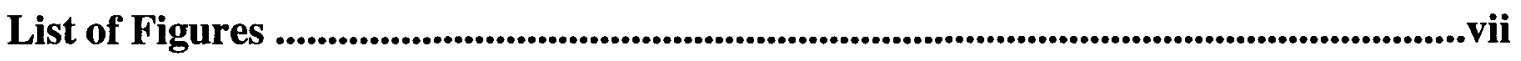

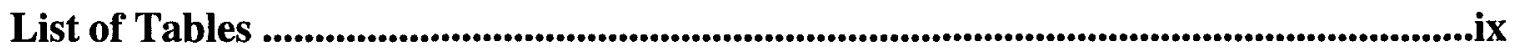

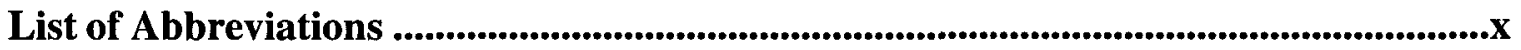

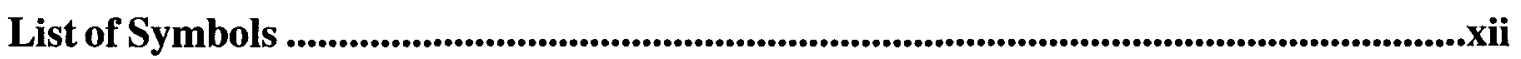

Chapter 1: Introduction ......................................................................................................1

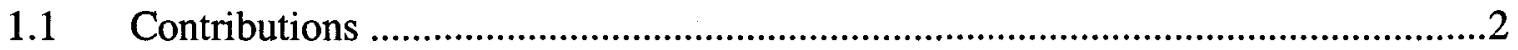

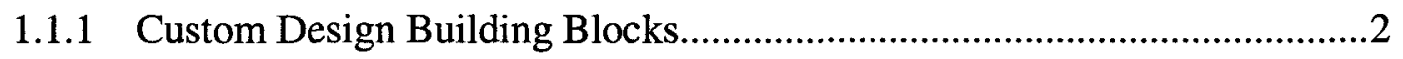

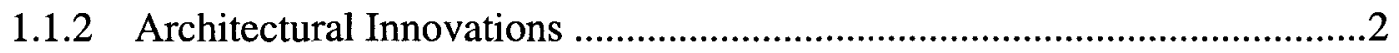

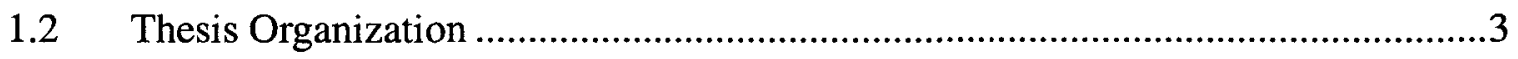

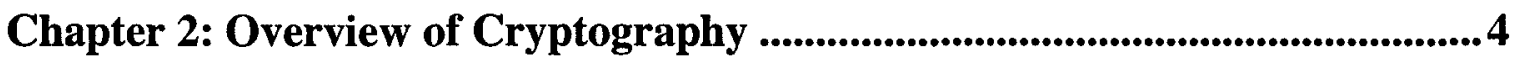

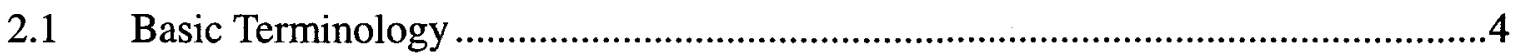

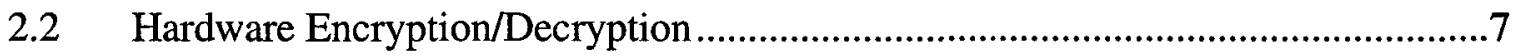

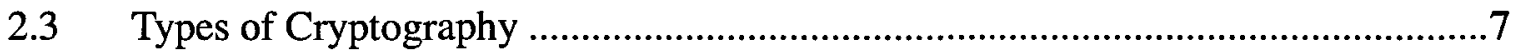

2.3.1 Symmetric-Key Cryptography .............................................................

2.3.2 Public-key Cryptography ...................................................................

2.3.2.1 Principles of Public-Key Cryptography......................................9

2.3.2.2 Characteristics of Public-Key Cryptography ............................. 11

2.3.2.3 Applications of Public-Key Cryptography ................................12

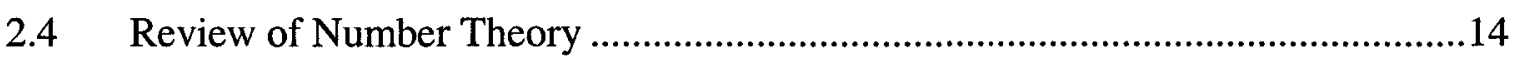




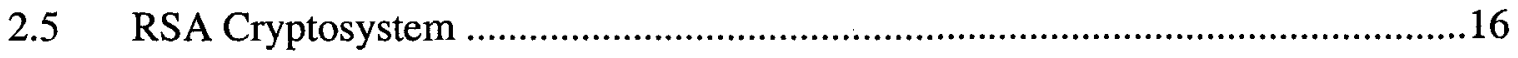

2.5.1 Computational Issues of the RSA Algorithm ..................................... 18

2.5.1.1 Modular Exponentiation ....................................................18

2.5.1.2 Efficient Modular Exponentiation ........................................19

2.5.1.3 Modified Exponentiation Algorithm ......................................21

2.5.1.4 Security Analysis of Exponentiation Algorithms .....................22

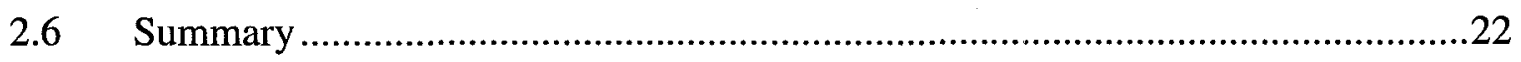

Chapter 3: Implementation of the Modular Multiplier .....................................23

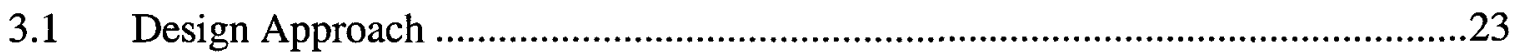

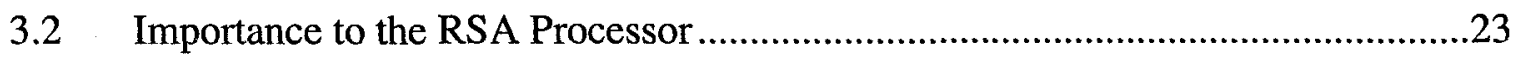

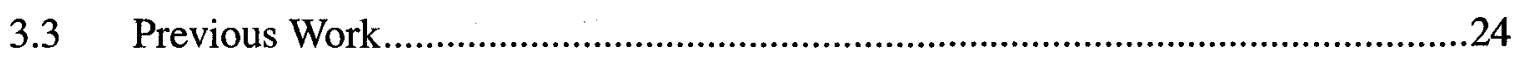

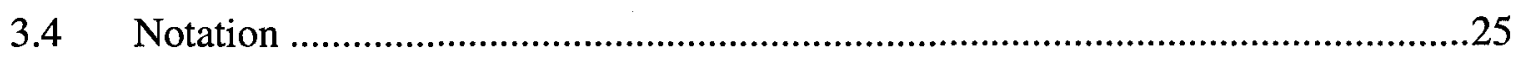

3.5 The Montgomery Modular Multiplication Algorithm ......................................25

3.5.1 Word Length of Input Parameters .......................................................27

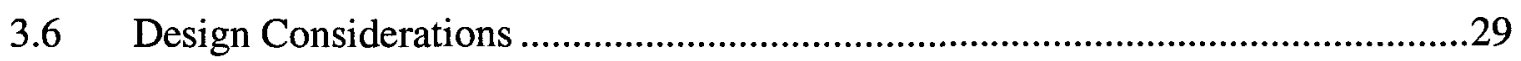

3.6.1 Large Number Addition and Redundant Representations ........................29

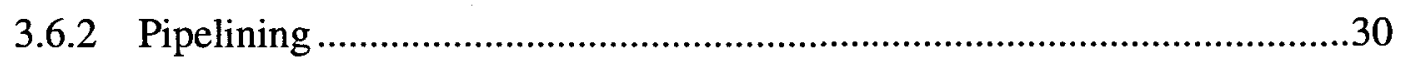

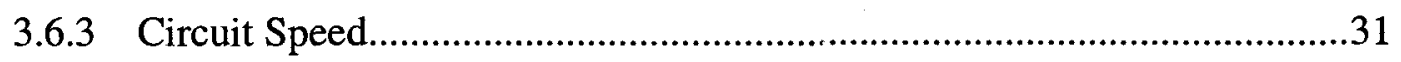

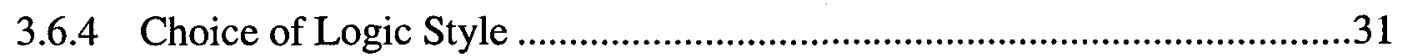

3.6.5 Data dependency of Montgomery's Algorithm (Recursive).....................32

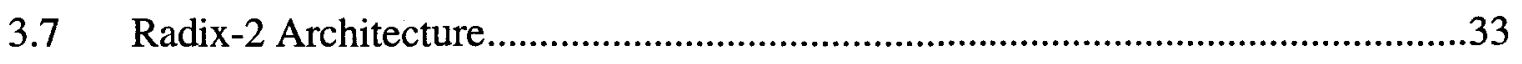

3.7.1 Modified Algorithm and Architecture .....................................................33

3.7.2 Data Dependency and Signal Flow Graphs ..........................................34

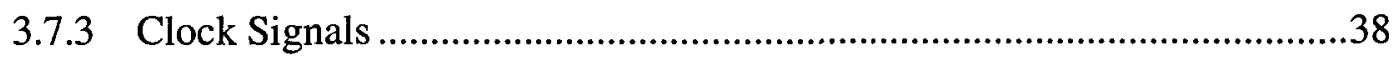

3.7.3.1 Single Phase Clock Signal ....................................................38 iv 
3.7.3.3 Unsuitability of Double Edge Triggered DFFs............................38

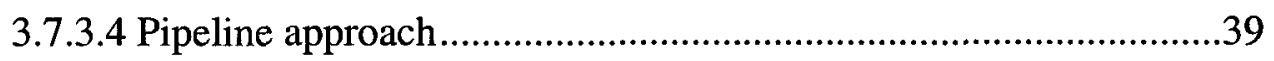

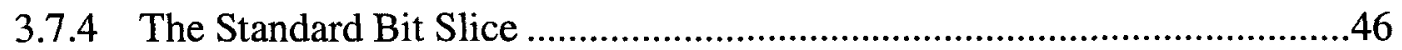

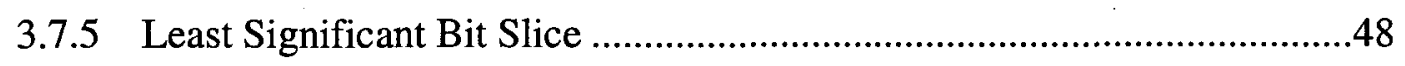

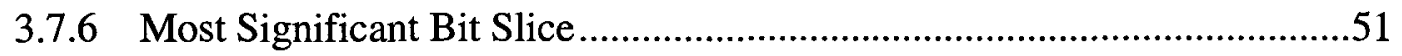

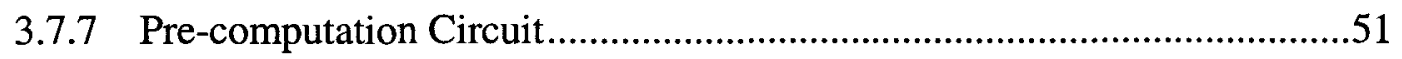

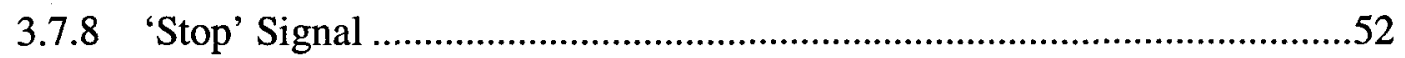

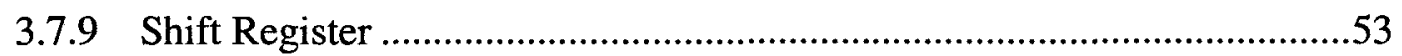

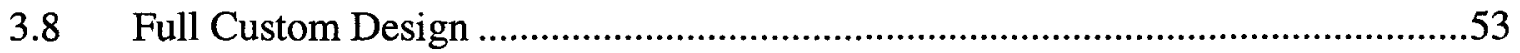

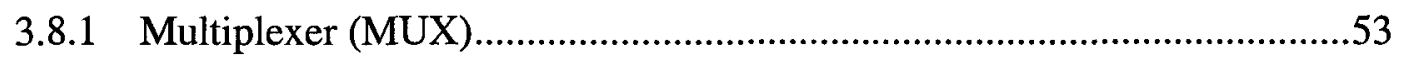

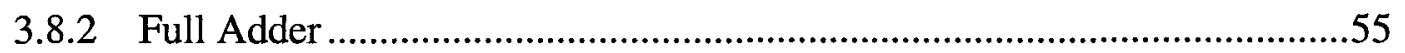

3.8.3 D Flip Flop and Clock Pulse Generators ...............................................58

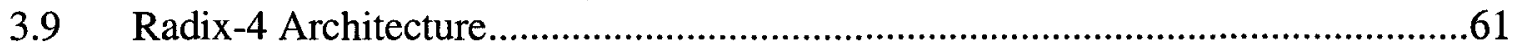

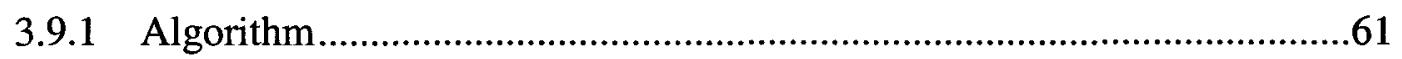

3.9.2 High Level Architecture ................................................................62

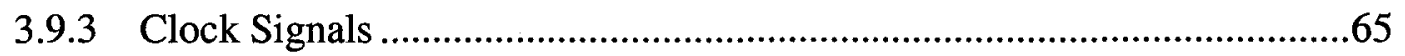

3.9.4 Radix-4 Standard Bit Slice.......................................................65

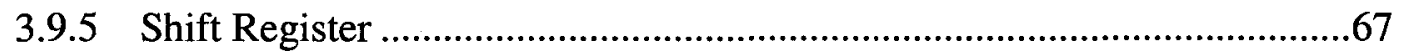

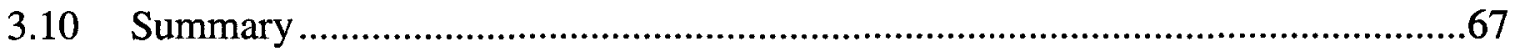

Chapter 4: Simulation Results and Comparison ..........................................68

4.1 Montgomery Modular Multiplier Primitives .................................................68

4.1.1 Pulsed D Flip Flop ...............................................................................68

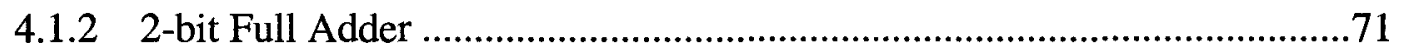

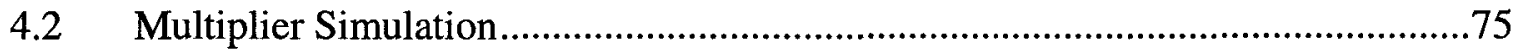

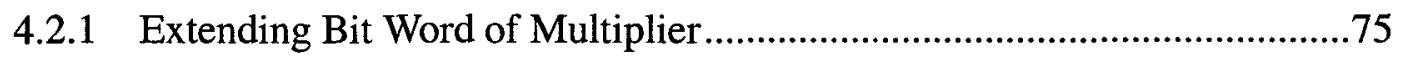




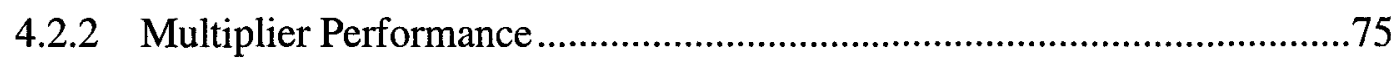

4.3 Comparison with Published Results .....................................................................76

4.3.1 Number of Clock Cycles and Maximum Clocking Speed............................76

4.3.2 Number of Transistors .............................................................................77

4.3.3 Power Consumption....................................................................................77

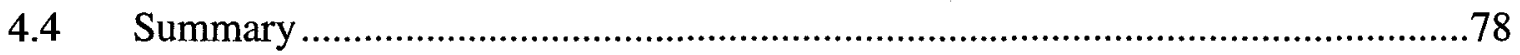

Chapter 5: Conclusion and Future Work ........................................................79

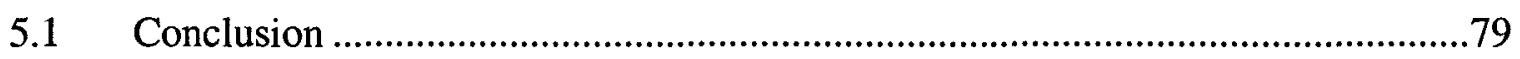

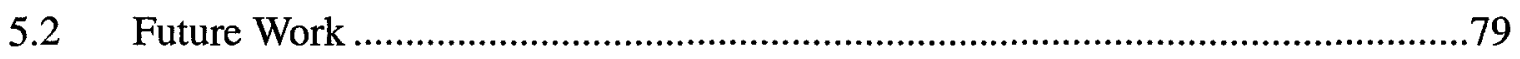

Bibliography ...................................................................................................................................................8

Appendix A: Self-duality Property of the Adder Functions....................................................83

Appendix B: 32-bit Modular Multiplier Transient Simulation.............................................84

Appendix C: Layout Diagrams of Multiplier Functional Blocks......................................91 


\section{List of Figures}

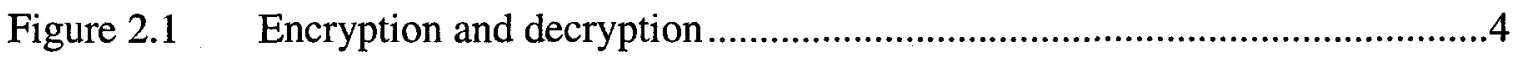

Figure $2.2 \quad$ Key management example ..........................................................13

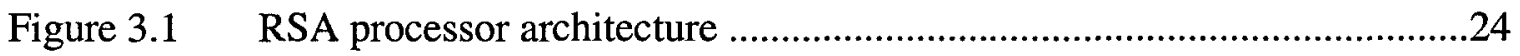

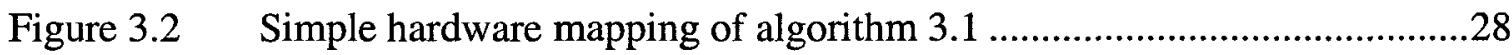

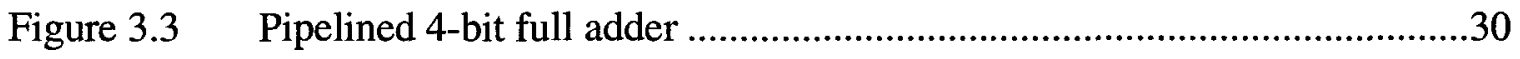

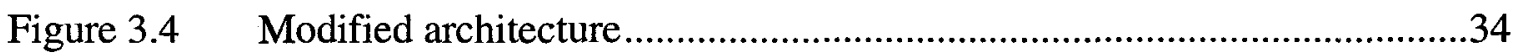

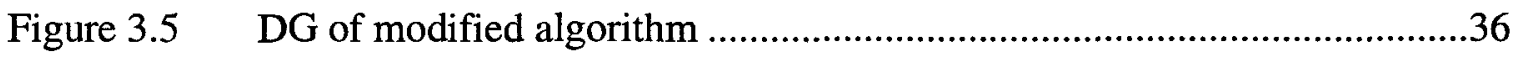

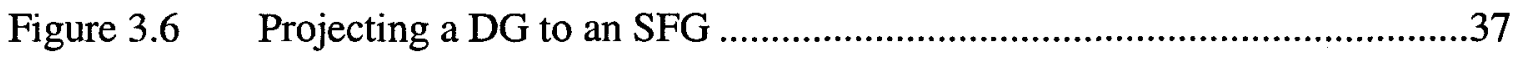

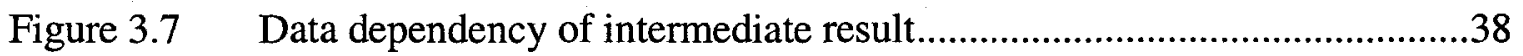

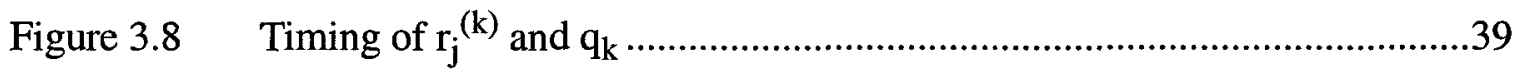

Figure 3.9 Adders with different pipeline approaches .......................................40

Figure 3.10 Three clocking approaches for pipeline stages ..................................41

Figure 3.11 Examples of three pipeline approaches ...........................................44

Figure 3.12 Hardware arrangement/complexity of each pipeline approach ................45

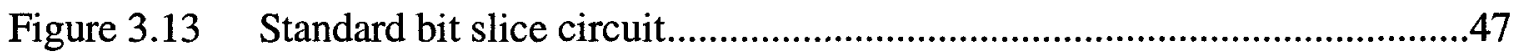

Figure 3.14 Least significant bit slice circuit .....................................................48

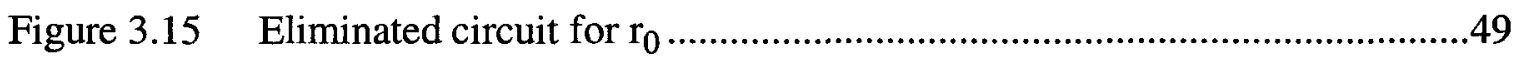

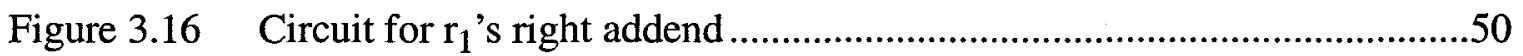

Figure 3.17 Most Significant Bit Slice ............................................................51

Figure 3.18 Pre-computation circuit...............................................................52

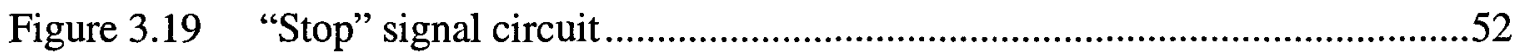

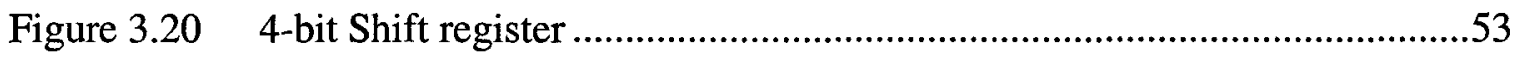

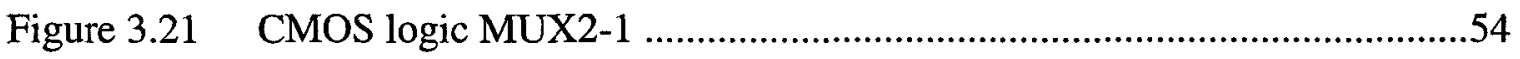

vii 


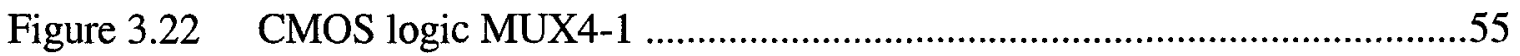

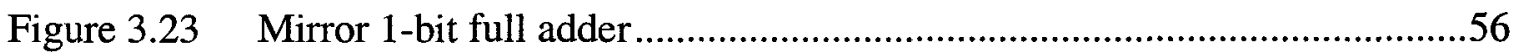

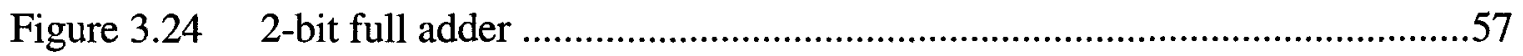

Figure 3.25 Implementation of 2-bit full adder with Boolean operators .....................58

Figure 3.26 Pulsed D Flip Flop and its pulse generators .......................................60

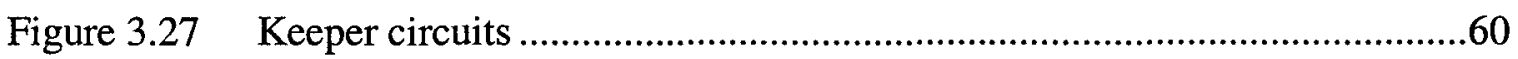

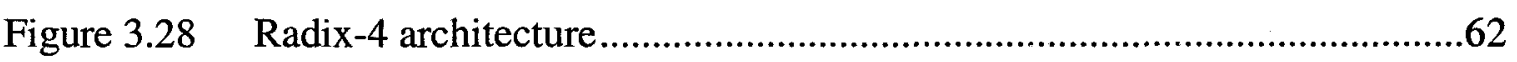

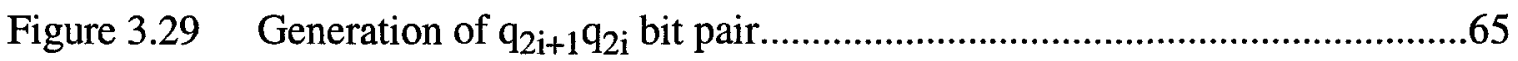

Figure 3.30 Standard bit slice of radix -4 architecture ............................................66

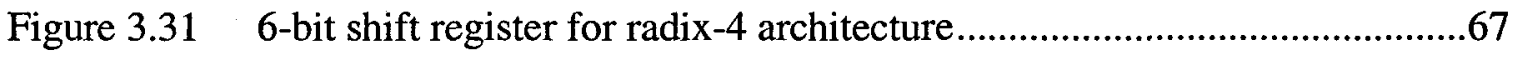

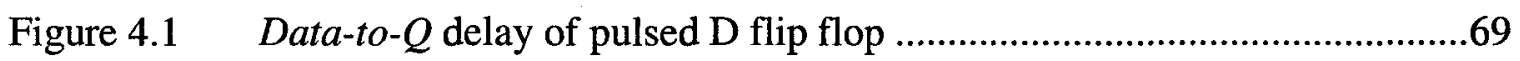

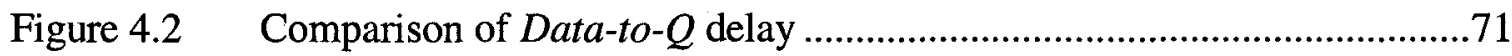

Figure 4.3 Carry circuit of 2-bit full adder before optimization ..............................72

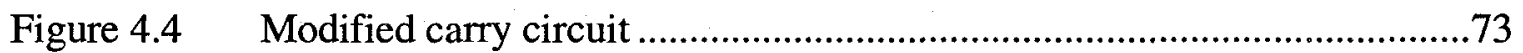

Figure 4.5 Time domain simulation of the 2-bit full adder (a) Standard cell library

1-bit FA cascade and (b) Optimized Adder...........................................74

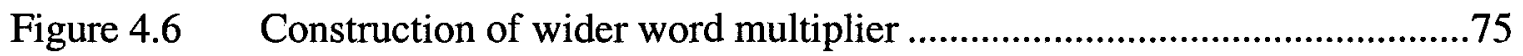




\section{List of Tables}

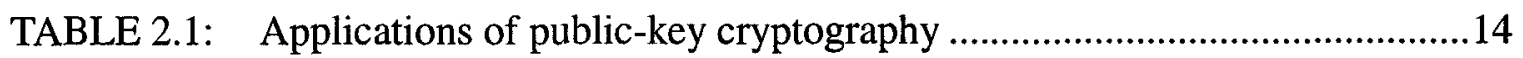

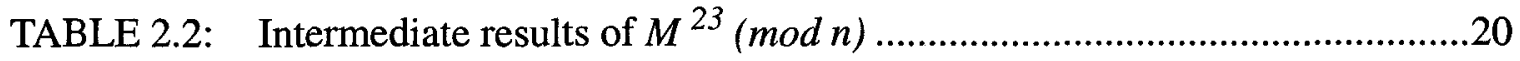

TABLE 3.1: Computing sequence of a pipelined 4-bit adder .....................................31

TABLE 3.2: Comparison of the three pipeline approaches ........................................46

TABLE 3.3: Relation between conditions and operation ........................................49

TABLE 3.4: Determination of $\mathrm{q}_{2 \mathrm{i}+1} \mathrm{q}_{2 \mathrm{i}}$ and the corresponding operations ..................63

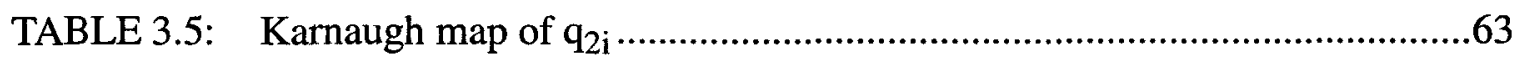

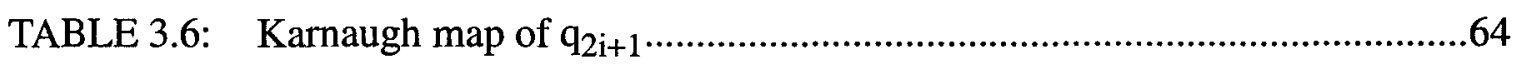

TABLE 3.7: Karnaugh map for variable A ….........................................................64

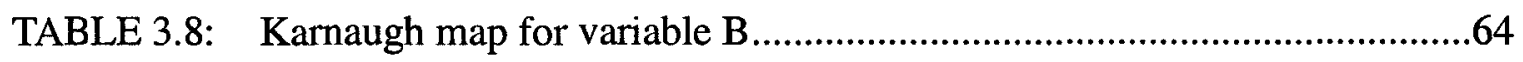

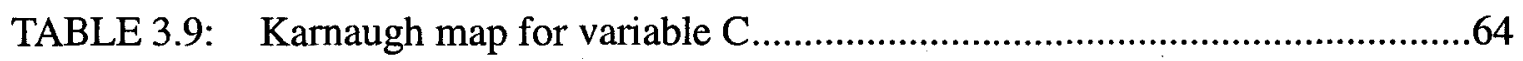

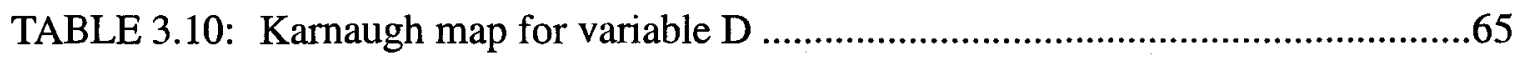

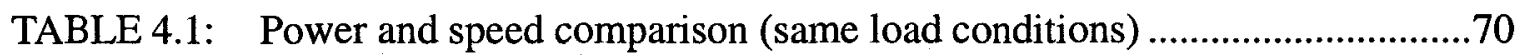

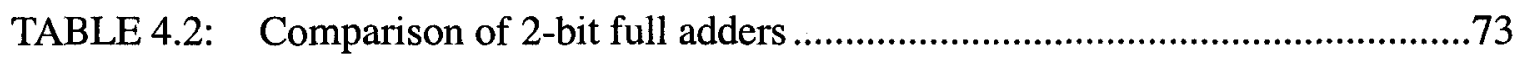

TABLE 4.3: $\quad$ Specifications of a 1024-bit multiplier (Radix-2) ...................................76

TABLE 4.4: $\quad$ Specifications of a 1024-bit multiplier (Radix-4) ...................................76

TABLE 4.5: Design comparisons in $0.35 \mu \mathrm{m}$ technology..........................................77

TABLE 4.6: Comparison of area and transistor count ...............................................78 


\section{List of Abbreviations}

\begin{tabular}{ll} 
RSA & Rivest, Shamir, Adleman \\
DSA & Digital Signature Algorithm \\
CMOS & Complementary Metal-Oxide-Semiconductor \\
DES & Data Encryption Standard \\
MUX & Multiplexer \\
VLSI & Very Large Scale Integration \\
FPGA & Field-Programmable Gate Array \\
LSB & Least Significant Bit \\
MSB & Most Significant Bit \\
DG & Dependence Graph \\
SFG & Signal Flow Graph \\
CPL & Complementary Pass Logic \\
DFF & D type Flip Flop \\
PDFF & Pulsed D type Flip Flop \\
FA & Full Adder \\
$T_{c q}$ & Clock-to-Q Delay \\
$T_{s e t u p}$ & Setup Time \\
$T_{d q}$ & Data-to-Q delay \\
$V_{d d}$ & Supply Voltage \\
\hline
\end{tabular}


$V_{t h p}$

$V_{t h n}$

PMOS transistor threshold voltage

NMOS transistor threshold voltage 


\section{List of Symbols}

D D flip flop

$\stackrel{1+1}{t}$ Multiplexer 4-1

2-bit Multiplexer 4-1

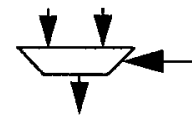

Multiplexer 2-1

D Positive edge triggered D flip flop

D Negative edge triggered D flip flop

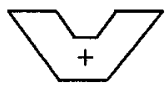

2-bit full adder

$+\quad$ 1-bit full adder/half adder 


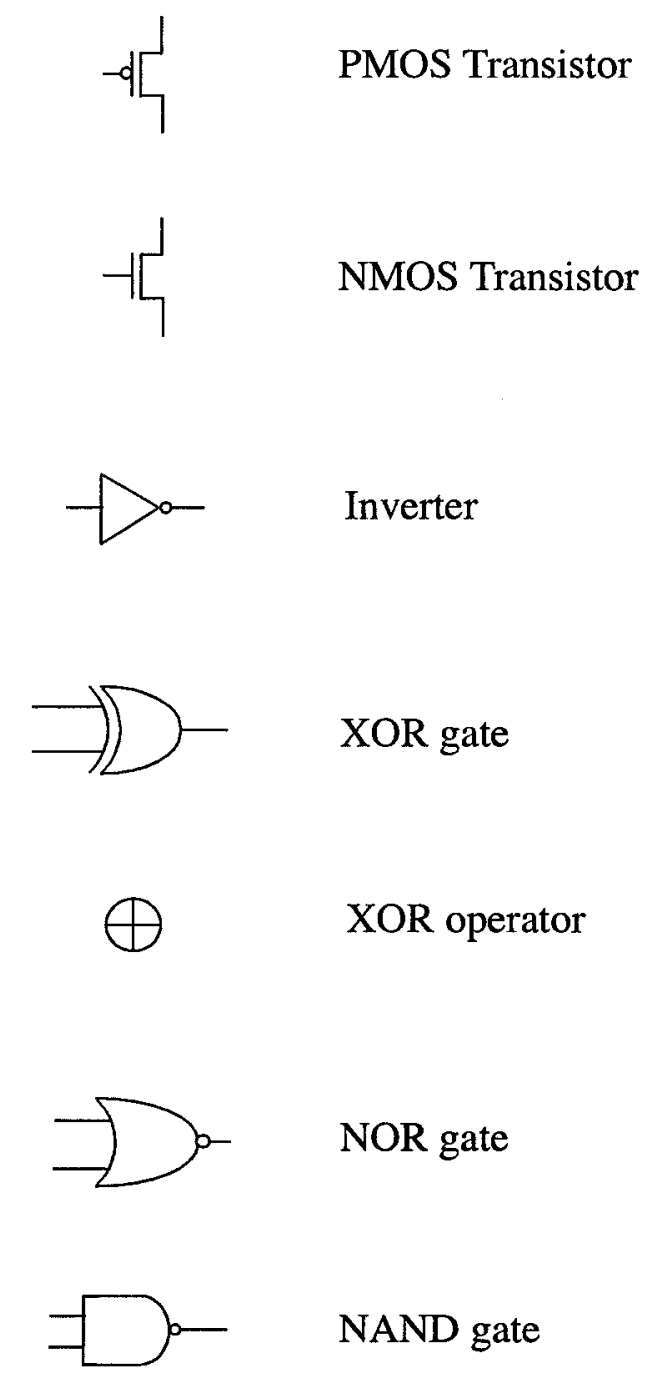

xiii 
The explosive growth of the Internet has been accompanied by a parallel evolution of security requirements, ones that are of particularly importance in the field of electronic commerce [1]. Of major importance are the means of maintaining data integrity, guaranteeing message authenticity and protecting against network-based attacks. Steadily maturing methods of cryptography and network security are applied to these needs [2].

The main goal of cryptography is one of enabling confidential data movement over insecure media. In general, there are two types of cryptosysytems, the first being secret-key cryptography, wherein a secret key is established and shared between peers. This key is used symmetrically for both encryption and decryption operations, and for this reason the general method often called symmetric cryptography.

The second type is called public-key cryptography [3]. In a public-key cryptosystem, a user has a pair of mathematically related keys: a public one that can be published without compromising system security, and a private one that is known only to its owner. The keys are such that deriving one from the other is computationally infeasible.

Both approaches have their advantages and disadvantages. Secret key cryptography is efficient but does not scale well because the number of participants is limited. Secondly, the message authentication made possible by a digital signature is not available. While public-key cryptography is untroubled by these problems, it has been highly inefficient, computationally. And if such a cryptosystem is used, the parties involved in a communication do not have to have met previously to share the secret key. Scalability and 
message authentication make public-key methods the ones of choice for modern e-business transactions.

Public-key algorithms are mathematically much more complex than their symmetric counterparts and therefore more time-consuming. For example, the famous 'RSA' [4] public-key algorithm (the letters are the initials of Rivest, Shamir and Adleman, the system inventors) is 1000 times slower than the Data Encryption Standard (DES) algorithm, a well known symmetric key algorithm, when implemented in hardware [5]. Some improvement is obviously called for, improvement that will take the form of a faster modular multiplier, as this is the core operation in most public-key systems.

\subsection{Contributions}

\subsubsection{Custom Design Building Blocks}

Logic gates such as the AND, OR, XOR and the Multiplexer (MUX), were implemented using conventional CMOS logic. Some more complex cells, such as 2-bit full adders use static designs. A custom digital counter was also designed. Critical path optimization involving a 2-bit full adder and a pulsed D flip-flop was also done.

\subsubsection{Architectural Innovations}

In order to reduce the number of clock cycles required to perform a multiplication, a reduced-latency architecture based on a radix-2 algorithm with an improved clocking scheme was devised. Latency here is defined as the number of clock cycles between the first clock when computation starts and the last clock when it completes. The new clocking method reduces the multiplication latency from $2 N$ to $1.25 N$ clocks for an $N$-bit pipelined modular multiply.

An alternative radix-4 architecture is also described in this document, the use of which results in a substantial reduction in the number of clock cycles needed to perform 
one modular multiplication. This speed improvement comes at the expense of a moderately silicon area and higher power consumption.

\subsection{Thesis Organization}

The remainder of this thesis is organized as follows:

- Chapter 2: Overview of cryptography.

- Chapter 3: Hardware architectures for the Montgomery modular multiplier and their building block implementations are described.

- Chapter 4: Simulation results and a comparison with other designs.

- Chapter 5: Concluding remarks and a proposal for future work.

- Appendices A, B, C and D contain auxiliary information. 
CHAPTER 2

\section{Overview of \\ Cryptography}

\subsection{Basic Terminology}

\section{Cryptosystem}

The plaintext to be encrypted consists of any human or machine-readable data. The encryption operation removes the contextual structure of the plaintext, so that while it remains machine-readable, it has been rendered meaningless. The encrypted message is called ciphertext [2].The process of recovering the plaintext from the ciphertext is called decryption. A cryptosystem refers to a set of cryptographic primitives used to provide security services [6]:

Figure 2.1 illustrates the basic model of a cryptosystem where a message intended for transmission over an unsecured channel is encrypted, sent and decrypted.

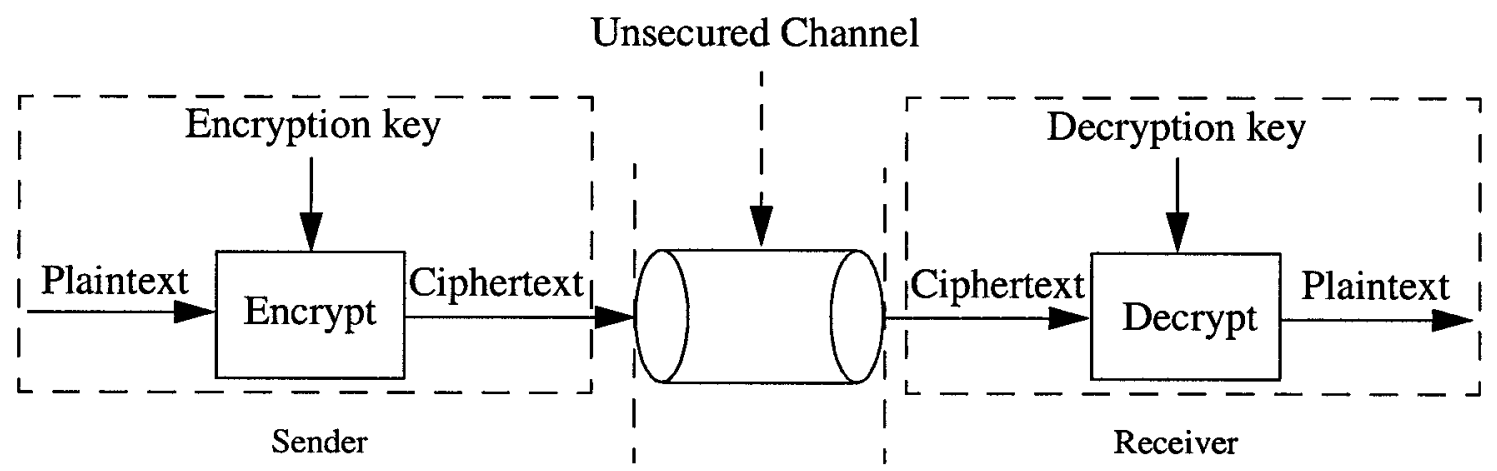

Figure 2.1 Encryption and decryption 


\section{Security goals}

The fundamental objectives of cryptography [6], [7] are:

1. Confidentiality: Keeping plaintext secret from unauthorized persons.

2. Data integrity: Ensuring that no unauthorized data modification has occurred.

3. Authentication: Ascertaining the source of data and the identity of an entity.

4. Non-repudiation: Preventing an entity from denying previous commitments or actions.

\section{Algorithms and Keys}

A cryptographic algorithm is the mathematical function used for encryption and decryption [5].

If the input of a cryptographic algorithm consists only of the plaintext or ciphertext to be encrypted or decrypted, the algorithm is called restricted. A second basic category is the modern cryptographic algorithm which usually has an extra input, a key, which might take on any of a large number of values. The main difference between restricted and modern algorithms is that the former's security depends upon algorithm secrecy whereas that of the latter upon key secrecy. (Modern algorithms are also called key-based algorithms.) The set of possible key values is called the keyspace.

If $M$ denotes the plaintext and $C$ the ciphertext, the encryption and decryption transformations are written

$$
\begin{aligned}
& C=E_{k}(M) \\
& M=D_{k}(C)
\end{aligned}
$$

Each uses a key, an encryption key and a decryption key respectively. If the two are identical or equivalent, the encryption system is symmetric. Otherwise it is an asymmetric or public-key method, in which case the keys are different but related. 


\section{Cryptanalysis}

Cryptanalysis refers to methods of recovering plaintext from ciphertext without knowing the decryption key. An attempted cryptanalysis is called an attack [5].

\section{Security of Encryption Algorithms}

The security of an encrypted message depends on its 'breakability'. An algorithm is unconditionally secure when the plaintext cannot be recovered no matter how much ciphertext is available to the cryptanalyst. The only methods which have this property are the so-called one-time pads. The keys for encryption and decryption are used only once, and the key length is not less than the length of plaintext. These methods are impractical for modern high-throughput cryptography and so are replaced with modern cryptography methods which are computationally secure. "Computationally Secure" means that it is possible to break the ciphertext, but only at a cost which greatly exceeds the ciphertext's value, so that an adversary will have no incentive to attack.

In summary, an encryption algorithm is deemed acceptable if it meets at least one of the following criteria [2]:

1. The cost of breaking the ciphertext surpasses the real value of the encrypted message.

2. The time spent to break the ciphertext exceeds the lifetime of the encrypted message.

\section{Protocols}

Cryptographic algorithms by themselves are limited in their ability to deal with security problems involving secrecy, authentication, integrity and outright dishonesty. But the robustness of a method can be greatly improved by making it part of a protocol, a sequence of predefined steps to be followed in dealing with a specified task. Two or more parties, who may be friends or adversaries, participate in a cryptology protocol, a well- 
designed one of which can prevent eavesdropping and cheating [5]. The use of protocols, enables encryption methods to be used in some very important applications, such as secure elections and digital cash.

\subsection{Hardware Encryption/Decryption}

Most encryption products take the form of specialized hardware. These hardware devices are inserted into a communications line and encrypt the stream of data passing through them. Although software encryption is becoming more and more popular, a hardware product is still the first choice for most military and commercial users. There are important reasons for this choice [5]. The first is speed. Encryption/decryption processes consist of many complicated operations on data which are not effectively implemented in software.

Another is security. An encryption algorithm running on a general-purpose computer has no physical protection in that an adversary can use debugging tools to surreptitiously modify even ROM-based programs. Hardware encryption devices can be securely encapsulated to prevent this - for example, a special-purpose VLSI chip can be coated with a chemical material which causes any attempt at accessing its interior to result in chip damage [5]. In addition, data-containing electromagnetic radiation is easily blocked. Shielding of computers, while possible, is much more difficult [5].

A third reason for the dominant position of hardware is ease of installation. It is easy and cheaper to protect telephone conversations, facsimile transmissions or data links by simply inserting a special-purpose encryption unit into a communications line than it is to design a microprocessor-based security system.

\subsection{Types of Cryptography}

Two general kinds of modern cryptography can be distinguished by the number of keys used: symmetric (single key or secret key) cryptography and asymmetric (two key or 
public key) ones. The fundamental operations found in these systems are implemented with secret key functions, public-key functions and hash functions ${ }^{1}$.

\subsubsection{Symmetric Key Cryptography}

Symmetric key cryptography, wherein the encryption and decryption keys are the same or equivalent, has a longer history than public-key cryptography. Symmetric methods are characterised by two participants having first to agree on a key and then communicating it to one another in some secure manner. This necessity of key sharing represents a drawback to the method, as it might be very difficult to achieve in practice. Furthermore, symmetric key cryptography cannot provide a non-repudiation service, as it is not possible to distinguish two participants sharing the same key [7].

On the other hand, the confusion ${ }^{2}$ and diffusion ${ }^{3}$ methods of symmetric key methods are very efficiently implemented on modern computers.

The most widely-used symmetric cryptosystem is IBM's DES [8], a worldwide standard for almost 30 years. Although its withdrawal from use has been recommended by the U.S. National Institute of Standards and Technology [9], it remains in common use, possibly because of inertia.

The DES is a 64-bit block cipher. A DES device accepts as input a plaintext block and a key and produces a 64-bit ciphertext block as output. To accomplish its ends, DES uses only standard arithmetic and logic operations on 64-bit numbers, and so is easily implemented in software. In addition, it is also suitable for hardware implementation because of its repetitive nature.

With secret key cryptography, serious difficulties arise when the number of users is increased: for every $n$ users, there are $\left(n^{2}-n\right) / 2$ potential pairs who may wish to commu-

1. A hash function maps a variable-length data block into a fixed-length value [2]. It can provide service of data integrity.

2. Obscures the relationship between the plaintext and the ciphertext [5].

3. Dissipates the redundancy of the plaintext by spreading it out over the ciphertext [5]. 
nicate privately, and an equal number of unique keys. It is unrealistic to expect either that a pair of users with no prior acquaintance will be able to wait for their unique key to be produced and then securely transmitted to them, or that all $\left(n^{2}-n\right) / 2$ pairs can be arranged in advance.

\subsubsection{Public-key Cryptography}

\subsubsection{Principles of Public-Key Cryptography}

The key multiplicity and management problems of secret key cryptography are consequent on the fact that keys can not be publicized. If they could be, a community of $n$ users would require only $O(n)$ keys, rather than $O\left(n^{2}\right)$. Attempts to deal with this difficulty have led to the development of public-key cryptosystems, so called because each user can publish a public key for use by any sender, but only this same user has access to the mathematically-related private key which can be used to recover the sender's plaintext. In the most popular of these, the RSA method, encryption and decryption are accomplished by methods which are mathematically-identical but which make use of different keys. In this sense, the two operations are functional inverses. It is unfeasible, using known methods, for a cryptanalyst to derive the private key from the public key.

The basic idea of the public-key cryptosystem was presented in the form of a challenge to the computing community by Diffie and Hellman in 1976 [3]. No actual algorithm was suggested by these authors. Soon afterwards (1977), Rivest, Shamir, and Adleman announced a specific solution to the Diffie/Hellman problem, the now wellknown RSA cryptosystem [4]. Since RSA, several more public-key systems have been proposed, ones differing in the manner of making the system secure. In particular, the security of an RSA cipher is due to the non-availability of efficient algorithms for factoring very large composite integers. Another example is the ElGamal Cryptosystem, where security is due to the computational intractability of discrete logarithms. 
It is conceptually helpful to think of a public-key system in terms of an abstraction called a trapdoor one-way function, or just a trapdoor function. In such a system, the encryption transformation $E: x \rightarrow f(x)$, where $x$ represents a block of plaintext, is relatively easy to do, i.e., is computationally feasible, but the inverse decryption transformation, where $x$ is recovered, $D: f(x) \rightarrow x$ is difficult in the extreme without subsidiary information. Functions $f(x)$ having this property are called one-way functions, and the subsidiary information needed to make transformation $D$ computationally feasible is called the trapdoor [3]. One can think of the route from $f(x)$ to $x$ as being a unique but very lengthy path through a maze, a path leading from the ciphertext back to the plaintext, and of the subsidiary information as a secret passage (trapdoor) directly connecting the two points and making a search through the maze unnecessary to the legitimate receiver.

The characteristics required of a trapdoor one-way function are easily stated formally [10]:

1. There exists an efficient method of computing $f(x)$ for all $x \in X$; where $X$ is the space of all possible message blocks, and

2. There is no computationally-efficient means of computing the inverse transformation $f^{-1}(y)$ for all $y \in f(X)$ in the absence of auxiliary information.

These can be restated in more-practical terms:

1. The sender must be able to perform the encryption transformation ( $E$, above) efficiently.

2. A cryptanalyst, legitimate or not, must be unable to perform the inverse transformation ( $D$, deducing the plaintext) in an efficient manner, when the only information available is the ciphertext.

3. The inverse transformation must become practical only when certain subsidiary information is available. 
A simple but important example of a one-way function is the multiplication of two large (about 100 decimal digits each) prime numbers. The inverse of this function is the recovery of the two prime factors from their 200 digit product. The factorization of a 200 digit composite is impossible for all intents and purposes. Large-integer multiplication is therefore a one-way function and could thus form the basis of a useful cryptology system if some way of augmenting it with a trapdoor capability could be found. This is in fact possible, and the RSA public-key cryptosystem is based on the result. The details of its development will be deferred until section 2.5.1, after some more remarks have been made about public-key cryptography in general and a brief review of the salient aspects of number theory has been presented.

\subsubsection{Characteristics of Public-Key Cryptography}

Public key methods exhibit important advantages over secret key ones:

First, the number of keys grows more slowly as the number of users is increased. Only $2 n$ keys, of which $n$ are published, are necessary for an $n$-user public-key system, numbers which are to be compared with the $n(n-1) / 2$ required in a symmetric-key cryptosystem having the same number of users.

Second, key management is easier than with symmetric key cryptography because secure transmission of secret keys in advance of message communication is unnecessary.

Third, public-key cryptography has the ability to provide a non-repudiation service, something which is not possible in symmetric systems.

Public-key systems do have a serious drawback in the form of slower processing. This makes them unattractive for high-throughput applications and in practice, the use of public-key systems is restricted to applications requiring functions the symmetric methods cannot provide, such as key exchange and digital signature. 


\subsubsection{Applications of Public-Key Cryptography}

A public-key cryptosystem application can be placed into one of three categories [3]:

1. Encryption/decryption: The sender encrypts a plaintext with the recipient's public-key. (This application is not suitable for large masses of plaintext because the encryption/decryption speed is much slower than that of a symmetric key method.)

2. Digital signature: A public-key algorithm can be used to produce a digital signature. If user $A$ wishes to send a message $M$ to user $B$, he encrypts $M$ using his own private key and sends the cipher to $B . B$ then decrypts the ciphertext by using $A$ 's public key. A successful decryption means that only $A$ could have sent the message, and so it has been effectively signed by $A$. In addition, it is impossible to alter the message without access to $A$ 's private key, so the message will have been authenticated both in terms of source and in terms of data integrity. This digital signature method provides the service of non-repudiation which is necessary in modern commercial transactions. But the message sent from $A$ is not secret, because anybody could use A's public key to decrypt it. A double encryption scheme, involving $A$ 's private key and $B$ 's public key, would be necessary to produce a message from $A$ to $B$ that is both secret and digitally signed.

3. Key management/exchange: Two sides of communication cooperate to exchange a session key to be used in a faster symmetric method. Several different approaches are possible, involving the private key(s) of one or both parties.

An example of key management is provided in [6] and illustrated in Figure 2.2. Each entity in the whole communication network has a key pair: public and private keys. Here it is assumed that the key generation process is absolutely secure. All of the public keys are stored in a published document analogous to the Yellow Pages. In this network, there are four entities or called participants labelled $U 1, U 2, U 3$ and $U 4 . M$ and $C$ refer to plaintext and ciphertext, respectively. The public keys are denoted $e_{U 1}$ and so forth. 
Suppose now that user $U 1$ has decided to send a message $M$ to $U 3$. The following steps will be followed:

1. U1 reads U3's public key from Public Document;

2. $U 1$ encrypts the message text: $C=E_{e_{U 3}}(M)$;

3. $U 1$ sends the ciphertext, $C$, to $U 3$;

4. $U 3$ decrypts the ciphertext: $M=D_{d_{U 3}}(C)$ to recover the plaintext, $M$.

The advantages of this approach are:

1. A trusted third party is not required.

2. The number of keys which must be kept secure is equal to the number of users, a highly-desirable characteristic when the number of users is large.

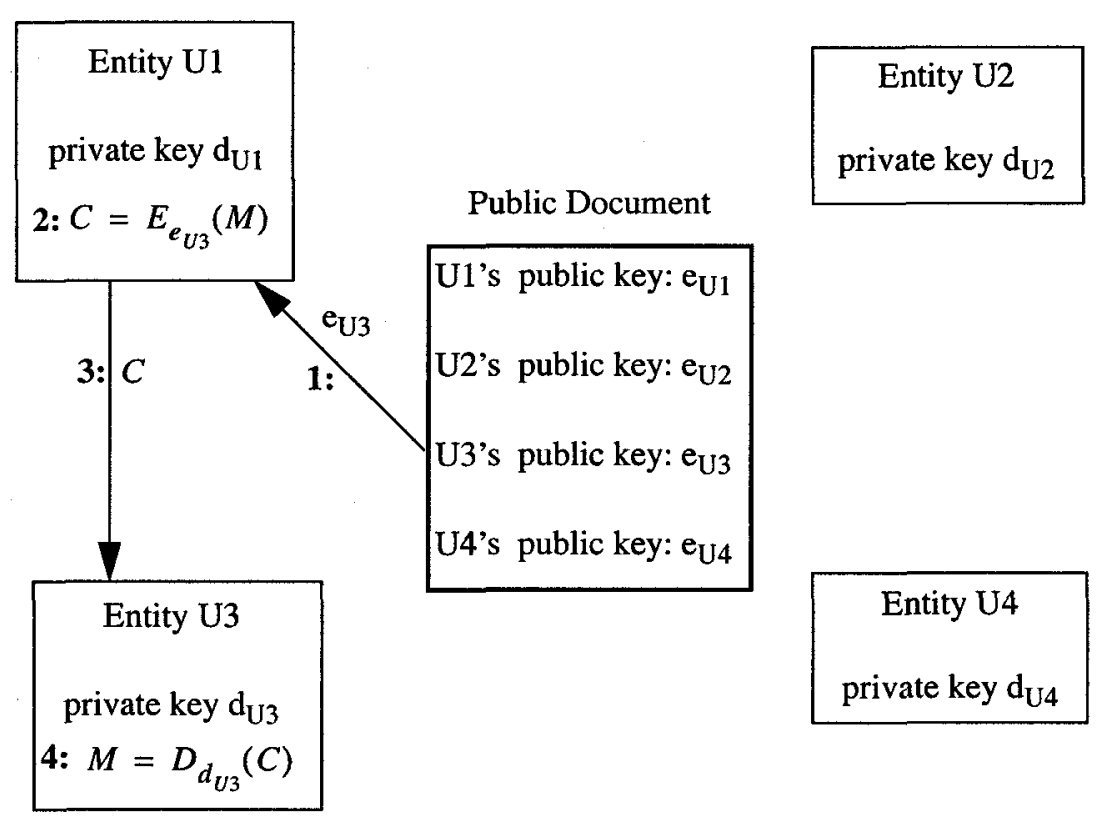

Figure 2.2 Key management example 
Not all public-key algorithms are suited to all three applications. Table 2.1 [2] presents a summary.

TABLE 2.1: Applications of public-key cryptography

\begin{tabular}{|l|l|l|l|}
\hline Algorithm & $\begin{array}{l}\text { Encryption/ } \\
\text { Decryption }\end{array}$ & Digital Signature & $\begin{array}{l}\text { Key Management/ } \\
\text { Exchange }\end{array}$ \\
\hline \hline RSA & Yes & Yes & Yes \\
\hline Diffie Hellman [3] & No & No & Yes \\
\hline ElGamal $[11]$ & Yes & Yes & Yes \\
\hline DSA* $[12]^{\text {No }}$ & Yes & No \\
\hline
\end{tabular}

*DSA (Digital signature algorithm, is based on the ElGamal signature algorithm)

\subsection{Review of Number Theory}

Some simple number-theoretic results must be understood before proceeding with the development of the RSA method.

\section{Prime and relatively prime numbers}

The positive integer 1 has just one positive divisor. Every other positive integer has at least two positive divisors, namely itself and unity. A prime is a positive integer greater than 1 that is divisible by no positive integers other than these.

For example, integers $2,3,17$, and 97 are primes.

The greatest common divisor of two positive integers $a$ and $b$, is the largest integer that divides both $a$ and $b$, and is written $\operatorname{gcd}(a, b)$. The integers $a$ and $b$ are called relatively prime if they share no common divisors greater than 1 . This is equivalent to saying that $a$ and $b$ are relatively prime if $\operatorname{gcd}(a, b)=1$.

Examples: 10 and 21 are relatively prime. 10 and 15 are not relatively prime because $\operatorname{gcd}(10,15)=5$. 


\section{Modular arithmetic, congruency}

If $a$ is an integer and $n$ is a positive integer, $a \bmod n$ is defined to be the remainder when $a$ is divided by $n$. For example, $11 \bmod 5=1,-10 \bmod 7=4$.

Two integers $a$ and $b$ are said to be congruent modulo $n$ if $a \bmod n=b \bmod n$. This is written as $a \equiv b \bmod n$.

The modular arithmetic operations of interest herein are:

1. Modular addition: $[(a \bmod n)+(b \bmod n)] \bmod n=(a+b) \bmod n$

2. Modular subtraction: $[(a \bmod n)-(b \bmod n)] \bmod n=(a-b) \bmod n$

3. Modular multiplication: $[(a \bmod n) \times(b \bmod n)] \bmod n=(a \times b) \bmod n$

In addition, exponentiation is performed by repeated multiplication, as in ordinary arithmetic.

\section{Multiplicative inverses}

$w$ is said to be the multiplicative inverse of $u$ if the product $u \times w$ is congruent to 1 modulo $n$, and is denoted $u^{-1}$.

\section{Euler's theorem}

The correctness of the RSA algorithm is based on Euler's theorem, one of the most famous results of number theory. Before introducing Euler's theorem, Euler's totient function should be presented first. For a positive integer $n$, Euler's totient function, also called Euler Phi-Function, $\phi(n)$, is the number of positive integers less than $n$ and which are relatively prime to $n$. If $n$ is a prime, all of the positive integers up to $n-1$ satisfy this condition and the totient is simply:

$$
\phi(n)=n-1
$$

Furthermore, if $n=p \times q$, where $p$ and $q$ are prime, it is

$$
\phi(n)=(p-1) \times(q-1)
$$


With these definitions in mind, Euler's theorem is stated: If $n$ is a positive integer and $a$ is an integer with $\operatorname{gcd}(a, n)=1$, then $a^{\phi(n)} \equiv 1(\bmod n)$.

\subsection{RSA Cryptosystem}

\section{RSA public-key scheme}

The first response to the Diffie/Hellman challenge came from Ronald Rivest, Adi Shamir, and Leonard Adleman at MIT and was first published in 1978 [4]. The RivestShamir-Adleman (RSA) scheme has since that time reigned supreme as the only widely accepted and implemented general-purpose approach to public-key encryption. RSA has withstood years of extensive cryptanalysis. Although the security of the RSA scheme has yet to be formally proved, there is a great deal of empirical evidence that this is so [2].

As has already been mentioned, the RSA scheme takes advantage of the intractability of the composite number factorization problem. In general, it is a block cipher in which a block of plaintext or of ciphertext takes the form of a large integer, having a value between 0 and $n-1$ for some $n$. The number of bits in one block will be less than or equal to $\log _{2}(n)$.

To establish oneself as an RSA user, the following steps are taken:

1. Randomly select two very large distinct primes $p$ and $q$.

2. Calculate $n=p \times q$, and $\phi(n)=(p-1) \times(q-1)$;

3. Randomly select the encryption key, $e$ which must satisfy $\operatorname{gcd}(\phi(n), e)=1$, and $1<e<\phi(n)$. That is, $e$ and $\phi(n)$ must be mutually prime. There exist efficient methods for determining mutual primality.

4. Use the extended Euclid algorithm [14] to compute the decryption key, $d$, which is the multiplicative inverse of $e$, modulo the totient value:

$$
d=e^{-1} \bmod \phi(n)
$$


5. Publish the pair $e$ and $n$ in the public-key directory. Senders will use this information to encrypt messages to be sent to you.

6. Keep $d$ and $\phi(n)$ secret. $d$ and $n$ will be used by you to decrypt messages prepared by others with your public key.

7. Discard $p$ and $q$ without disclosing them.

The generated keys are used as follows:

Once again, the plaintext block is denoted by $M$ and the ciphertext block by $C$ : key:

The sender transforms the plaintext to the ciphertext using the recipient's public

$$
C=M^{e}(\bmod n)
$$

The recipient recovers the plaintext using his private key:

$$
C^{d}(\bmod n)=\left(M^{e}\right)^{d}(\bmod n)=M^{e d}(\bmod n)
$$

Since we have $e d=k \cdot \phi(n)+1$, according to Euler's theorem, we can obtain

$$
M^{k \cdot \phi(n)+1} \bmod n=\left(M^{\phi(n)}\right)^{k} \cdot M \bmod n=1^{k} \cdot M \bmod n=M
$$

The modular exponentiations in both transformations are carried out with a series of modular multiplications, where the property

$[(a \times b) \bmod n]<n$

keep the word length of the intermediate product under control. (See next section.) One thing to be noted is that the decryption cannot be done without knowing $d$. $d$ can in principle be derived from the published value of $n$, but the task is computationally unfeasible, as this involves the factoring of a very large composite number. The RSA procedure thus satisfies the requirements for a public-key system laid out by Diffie and Hellman.

It can be noted that people have been investigating the said factorization for more than two thousand years, but the record is still only 193 decimal-digit bits (640 binary bits) reported by [15]. The number factored was a product of two 97 decimal-digit primes, and 
according to the report, the effort took approximately $302.2 \mathrm{GHz}-$ Opteron-CPU years, over five months of calendar time [15].

\subsubsection{Computational Issues of the RSA Algorithm}

\subsubsection{Modular Exponentiation}

Consider modular exponentiation: $C=M^{e}(\bmod n)$.

The conceptually simplest way would be to simply compute $M^{e}$ and then perform a division to obtain the remainder

$C=M^{e}-k n$, where $k$ is the quotient of $M^{e}$ divided by $n$.

But the intermediate result $M^{e}$ would be of enormous size. Assuming both $M$ and $e$ have 512 bits each, in order to store the $M^{e}$, a

$$
\log _{2}\left(M^{e}\right) \approx\left(512 \times 2^{512}\right)=2^{521}
$$

bit memory is necessary. It is impossible to build a computer with such a huge memory.

But according to the property of modular arithmetic

$$
[(a \bmod n) \times(b \bmod n)] \bmod n=(a \times b) \bmod n
$$

the calculation can be accomplished as a series of modular multiplications, and all of the intermediate quantities will be smaller than $n$. This makes the calculation of modular exponentiation practical.

For example, the calculation of $6^{7}$ mod 13 involves the following series of intermediate magnitudes:

$$
\begin{aligned}
& 6 \bmod 13=6 \\
& 6 \times 6 \bmod 13=36 \bmod 13=10 \\
& 6^{3} \bmod 13=6 \times 6^{2} \bmod 13=6 \times 10 \bmod 13=8 \\
& 6^{6} \bmod 13=6^{3} \times 6^{3} \bmod 13=8 \times 8 \bmod 13=12
\end{aligned}
$$




$$
6^{7} \bmod 13=6^{6} \times 6 \bmod 13=12 \times 6 \bmod 13=7
$$

The end result is seen to be correct on doing the arithmetic directly, giving

$$
6^{7}=279936=21533 \times 13+7 .
$$

\subsubsection{Efficient Modular Exponentiation}

The previous exponentiation involved numbers that are very small compared to those which would be seen in an RSA operation and so computational efficiency was not an issue - but it is in practical RSA work, and so an efficient method for performing exponentiations involving very large integers (512 bits, or 1024 bits) must be found. It would be infeasible to compute $C=M^{e}(\bmod n)$ by performing $e-1$ successive modular multiplications of $M$.

If $e$ is 512 bits, this approach requires nearly $2^{512}$ modular multiplications. This is not acceptable in modern cryptosystem applications. The binary method [14] for exponentiation can reduce the iteration time from $2^{512}$ to $t$, where $t$ is the number of 1 's in $e^{\text {'s }}$ binary representation. The binary method scans the bits of the exponent either from left to right or from right to left. The former is called left-to-right algorithm and the later is called the right-to-left algorithm.

The binary expression of $E$ is

$E=\left(e_{k-1} e_{k-2} \cdots e_{1} e_{0}\right)=\sum_{i=0}^{k-1} e_{i} 2^{i}$, for $e_{i} \in\{0,1\}$.

The left-to-right binary method for computing $C=M^{E}(\bmod n)$ is given below [16]:

Input: $M, E=\left(e_{k-1}, \ldots, e_{0}\right)_{2}$, and $n$;

Output: $C=M^{E}(\bmod n)$;

Algorithm:

$\left\{1\right.$. If $e_{k-1}=1$ Then $C=M$ Else $C=1$; 


\section{For $i=k-2$ Downto 0\{}

$2.1 C=C \times C(\bmod n)$;

2.2 If $e_{i}=1$ Then $C=C \times M(\bmod n)$;

\section{\}}

\section{Return C}

\}

For example, let $E=23$, which implies $E=(10111)_{2}$. We want to calculate $M^{E}$

TABLE 2.2: Intermediate results of $M^{23}(\bmod n)$

\begin{tabular}{|l|l|l|l|}
\hline$i$ & $e_{i}$ & After step 2.1 & After step 2.2 \\
\hline \hline 3 & 0 & $M^{2}(\bmod n)$ & $M^{2}(\bmod n)$ \\
\hline 2 & 1 & $M^{4}(\bmod n)$ & $M^{5}(\bmod n)$ \\
\hline 1 & 1 & $M^{10}(\bmod n)$ & $M^{11}(\bmod n)$ \\
\hline 0 & 1 & $M^{22}(\bmod n)$ & $M^{23}(\bmod n)$ \\
\hline
\end{tabular}

using the left-to-right algorithm. Table 2.2 lists the procedure of the calculation.

Another attractive exponentiation algorithm is Montgomery's powering ladder [17]. It is given below:

Input: $M, E=\left(e_{k-1}, \ldots, e_{0}\right)_{2}$ and $n$;

Output: $C=M^{E}(\bmod n)$;

Algorithm:

\{1. $C_{0}=1 ; C_{1}=M$;

2. For $i=k-1$ Downto 0

2.1 If $\left(e_{i}=0\right)$ Then

$$
\left\{C_{1}=C_{0} \cdot C_{1} \bmod n ; C_{0}=\left(C_{0}\right)^{2} \bmod n ;\right\}
$$


2.2 Else $\left[\left(e_{i}=1\right)\right]$

$$
\left\{C_{0}=C_{0} \cdot C_{1} \bmod n ; C_{1}=\left(C_{1}\right)^{2} \bmod n ;\right\}
$$

\section{Return $C_{0}$;}

\}

\subsubsection{Modified Exponentiation Algorithm}

The Montgomery modular multiplication algorithm computes $A \times B \times 2^{-k} \bmod n$, where $k$ is the maximum length of $A, B$ and $n$. It can not be directly applied to modular exponentiation because of the extra factor of $2^{-k}$. However, the addition of pre- and postcomputation stages fixes this problem. One obtains a modified Montgomery powering ladder algorithm:

Input: $M, E=\left(e_{k-1}, \ldots, e_{0}\right)_{2}, n$ (assume $\left.M, E, n<2^{k}\right)$;

Function: $\operatorname{Mont}(A, B)=A \times B \times 2^{-k} \bmod n$;

Output: $C=M^{E}(\bmod n)$;

Algorithm:

$\left\{1 . C_{0}=\operatorname{Mont}\left(1,2^{2 k}\right) ; C_{1}=\operatorname{Mont}\left(M, 2^{2 k}\right) ; / /\right.$ Pre-computation

2. For $i=k-1$ Downto 0

2.1 If $\left(e_{i}=0\right)$ Then

$$
\left\{C_{1}=\operatorname{Mont}\left(C_{1}, C_{0}\right) ; C_{0}=\operatorname{Mont}\left(C_{0}, C_{0}\right) ;\right\}
$$

2.2 Else $\left[\left(e_{i}=1\right)\right]$

$$
\left\{C_{0}=\operatorname{Mont}\left(C_{1}, C_{0}\right) ; C_{1}=\operatorname{Mont}\left(C_{1}, C_{1}\right) ;\right\}
$$

3.1. $C_{0}=\operatorname{Mont}\left(C_{0}, 1\right) ; / /$ Post-computation

\subsection{Return $C_{0}$;}


In the above algorithm, $C_{0}$ and $C_{1}$ include a factor of $2^{k}$ after the pre-computation step. In the computation of step $2, C_{0}$ and $C_{1}$ have an unchanged factor of $2^{k}$. In the postcomputation step 3.1, the factor $2^{k}$ appearing in $C_{0}$ is removed by multiplying $C_{0}$ and 1 .

\subsubsection{Security Analysis of Exponentiation Algorithms}

That sensitive information can leak from cryptographic devices via side-channel attacks has been validated by research in recent years. Side-channel attacks are based on the fact that some side-channel information, for example, timing and/or power, depends on the data being processed and/or instructions being executed [18]. Both the left-to-right method and the right-to-left method contain conditional branches, for example, If statements, which is driven by secret data, for example, a private key. Secret data may also be compromised by a side-channel analysis based on differing behaviors of the two branches [18]. The regularity of the Montgomery powering ladder provides an inherent protection against this type of attack, unlike binary methods [18].

More information regarding the Montgomery powering ladder can be found in $[17][18]$.

This exponentiation algorithm can be used as the basis of an RSA processor.

\subsection{Summary}

A brief introduction to cryptography has been provided, with the main emphasis being on public-key cryptography in general and the RSA method in particular. These methods are powerful tools in modern e-business.

Because of the importance of modular exponentiation in public-key cryptosystems, an efficient implementation of this operation was also described, one which is insensitive to side-channel and other implementation attacks. 


\section{Modular Multiplier}

In this chapter, the hardware implementations of both radix-2 (section 3.4 to 3.7 ) and radix-4 (section 3.9) modular multipliers are described. Some design issues occurring at both the architectural and gate levels are discussed.

\subsection{Design Approach}

This design process taken was completed using a top-down approach where a modular multiplier based on the Montgomery algorithm was decomposed into subsystems that were further decomposed into simpler subsystems and so forth until a level is reached at which the subsystems could be realized directly with available modules [19]. At the lowest (transistor) level a full custom design strategy was followed to build better performing logic gates, full adders and registers requiring lower supply voltages. The designtime penalty for doing things this way was justified by the results.

A $0.18 \mu \mathrm{m}$ CMOS process was used to realize circuitry that can operate over a range of supply voltages, from the standard $1.8 \mathrm{~V}$ to as low as $1.0 \mathrm{~V}$.

\subsection{Importance to the RSA Processor}

Modular multiplication is the core arithmetic operation of most public key algorithms such as RSA, ElGamal [11], DSA [12], and Diffie-Helman [3], so fast and efficient modular multiplication is the key to real-time public key abilities.

Figure 3.1 is a block diagram of an RSA processor used to perform this basic function $\left(\operatorname{Result}=M^{E} \bmod N\right)$, that is used in both encryption and decryption operations. 


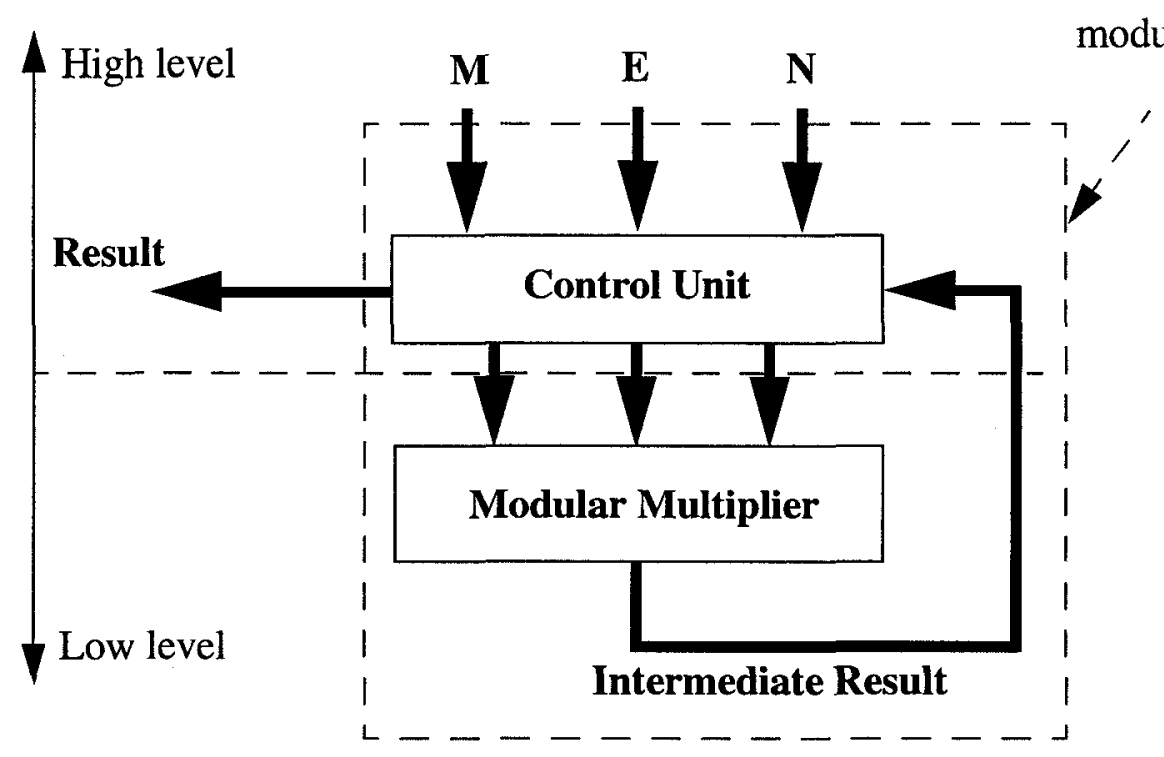

Figure 3.1 RSA processor architecture

In Figure 3.1, $M$ is either the plaintext or the ciphertext. $N$ is the modulus and $E$ either the public or the private key. The modular multiplier can be considered as a lower level component which performs modular multiplication. The Control Unit issues the signals which govern data movement and control the iteration. The RSA and other methods mentioned differ only in the appearance to the user of the control unit, so that the results of this thesis will be usable in all of them, but only the RSA method will be discussed in detail.

\subsection{Previous Work}

In this work, we will concentrate on modular multiplication using the Montgomery algorithm. Other ones based on the Brickell algorithm are not discussed in this thesis.

Many researchers have reported hardware implementations of the Montgomery algorithm, several of which were effected with FPGAs. The choice of an FPGA platform results in short design times and flexible architectures. But there is no such thing as a free lunch - FPGA-based multipliers tend to be too slow for real-time work. 
Other implementations use the ASIC concept. Walter [20] presented a systolic array modular multiplier in 1993 and similar structures are due to other authors $[21][22][23][24]$. All of these suffered from long latencies resulting from the 'one clock gap' property of Montgomery's algorithm [25] (See section 3.6.3). One mod-N multiplication required $3 N$ clock cycles. Then Tsai ([25]) reduced this figure to $1.5 N$ clocks using a 'non-interlaced' systolic array architecture. In this thesis, a yet-newer approach is presented, one which gets the job done with only $1.25 \mathrm{~N}$ clocks at the cost of a critical-path lengthening. The effect on multiplication time of the increased logic depth was compensated by carefully optimizing a fully custom circuit design to achieve a critical path delay which was actually less than those of previously reported designs.

\subsection{Notation}

The RSA algorithm assumes an $\mathrm{N}$-digit positional numbering system wherein a digit sequence has the meaning $X=\sum_{n=0}^{N-1} x_{n} r^{n}$, where $0 \leq x_{n}<r$, and $r$ is called the base or radix. In particular, if $r$ is 2 , one has the familiar binary system. In this thesis, a capital letter is usually used to represent a number and a subscripted lowercase one to represent the individual digits. In the binary system, the digits (bits) have two possible values, 0 and 1. For example, if $A$ is an $N$-bit binary number, one has $A=\sum_{n=0}^{N-1} a_{n} 2^{n}, a_{n} \in[0,1]$.

Reference must sometimes be made to the result of the $n^{\text {th }}$ iteration of some quantity. Parenthesized superscripts are used in such cases, superscripting that must not be confused with exponentiation; that is $R^{(n)} \neq R^{n}$, for example.

\subsection{The Montgomery Modular Multiplication Algorithm}

A 'straightforward' modular multiplication operation uses a division to determine the quotient which is to be multiplied by the modulus. Montgomery's method [26] avoids this time-consuming operation. The quotient depends upon the least significant bit of the intermediate result (see step 3 of Algorithm 3.1). This property is very useful to the 
designer of a pipelined bit serial multiplier since the next iteration can be started as soon as this bit has become available. If the multiplier is $A=\sum_{n=0}^{N-1} a_{n} 2^{n}$ and the multiplicand is $B$, the product $A \times B$ is accumulated as the sum of left-shifted values of $B$, the bits of $A$ being used to control the decision whether to include the current shifted $B$ in the sum. Residue reduction is used at each step to keep the size of the accumulated sum less-than or equal-to $N$ bits. A pipelining technique is used to hasten the process.

Montgomery's algorithm performs modular multiplication in two steps per bit: the product $R^{(i)}=a_{i} \times B+R^{(i-1)}$ is first generated, and in the second step is reduced modulo $M$.

Montgomery's algorithm will work in any base, but for the special case of radix-2, it can be written as follows:

\section{Algorithm 3.1 [27] (Radix-2)}

Input:

Modulus: $\mathrm{M}(\mathrm{N}$ - bit, but two MSBs=00)

Multiplier: $\mathrm{A}(N$ - bit, but $\mathrm{MSB}=0)$

Multiplicand: $\mathrm{B}(N$ - bit, but $\mathrm{MSB}=0)$

Output:

Result: $R=A \times B \times 2^{-N} \bmod M$

\section{Algorithm:}

\{1. $\mathrm{R}^{(-1)}=0$;

\section{For $\mathrm{i}=0$ To $\mathrm{N}-1$ \{}

3. $\mathrm{q}_{\mathrm{i}}=\left(\mathrm{R}^{(\mathrm{i}-1)}+\mathrm{a}_{\mathrm{i}} \times \mathrm{B}\right) \bmod 2$; 
4. $\quad \mathrm{R}^{(\mathrm{i})}=\left(\mathrm{R}^{(\mathrm{i}-1)}+\mathrm{a}_{\mathrm{i}} \times \mathrm{B}+\mathrm{q}_{\mathrm{i}} \times \mathrm{M}\right) / 2$;

\}

5. Return $\mathrm{R}^{(\mathrm{N}-1)}$;

\}

\subsubsection{Word Length of Input Parameters}

The sequence of $R^{(i)}$ generated by this algorithm is

$2 \times R^{(0)}=R^{(-1)}+a_{0} \times B+q_{0} \times M=a_{0} \times B+q_{0} \times M$

$2 \times R^{(1)}=R^{(0)}+a_{1} \times B+q_{1} \times M=\left(a_{0} \times B+q_{0} \times M\right) / 2+a_{1} \times B+q_{1} \times M$

$=>4 \times R^{(I)}=a_{0} \times B+q_{0} \times M+2 \times\left(a_{1} \times B+q_{1} \times M\right)=\left(a_{1} a_{0}\right) \times B+\left(q_{1} q_{0}\right) \times M$

When the initial condition $R^{(-1)}$ is included, this is

$2 \times R^{(0)}=a_{0} \times B+q_{0} \times M$

$4 \times R^{(1)}=\left(a_{1} a_{0}\right) \times B+\left(q_{1} q_{0}\right) \times M$

$8 \times R^{(2)}=\left(a_{2} a_{1} a_{0}\right) \times B+\left(q_{2} q_{1} q_{0}\right) \times M$

$2^{N} \times R^{(N-1)}=A \times B+Q \times M$

(Here, $A=a_{N-1} a_{N-2} a_{N-3} \ldots a_{2} a_{1} a_{0}, Q=q_{N-1} q_{N-2} q_{N-3} \ldots q_{2} q_{1} q_{0}, a_{i}$ and $q_{i} \in(0,1)$ )

The final result is $R^{(N-1)}=A \times B \times 2^{-N} \bmod M$.

From the Equation (3.1), it can be seen that if $A$ and $B$ are $N-1$ bit words, their product $A \times B$ will occupy at most $2 N$-2 bits. If $M$ occupies an $N$-2 bit word, and $Q$ an $N$-bit word, then the product $Q \times M$ will be no longer than $2 N-2$ bits. Therefore the right hand side is $2 N-1$ bits in length at most. This length guarantees the length of $R^{(N-1)}$ in the left hand side to be at most $N-1$ bits. 
Equation (3.1) shows that after the input range condition has been imposed, the output of the modular multiplication algorithm can be used directly as input to the same algorithm without additional processing [28], a very desirable characteristic when implementing of modular exponentiation as a sequence of modular multiplications.

The 'obvious' hardware realization of Algorithm 3.1 is illustrated in Figure 3.2 [27].

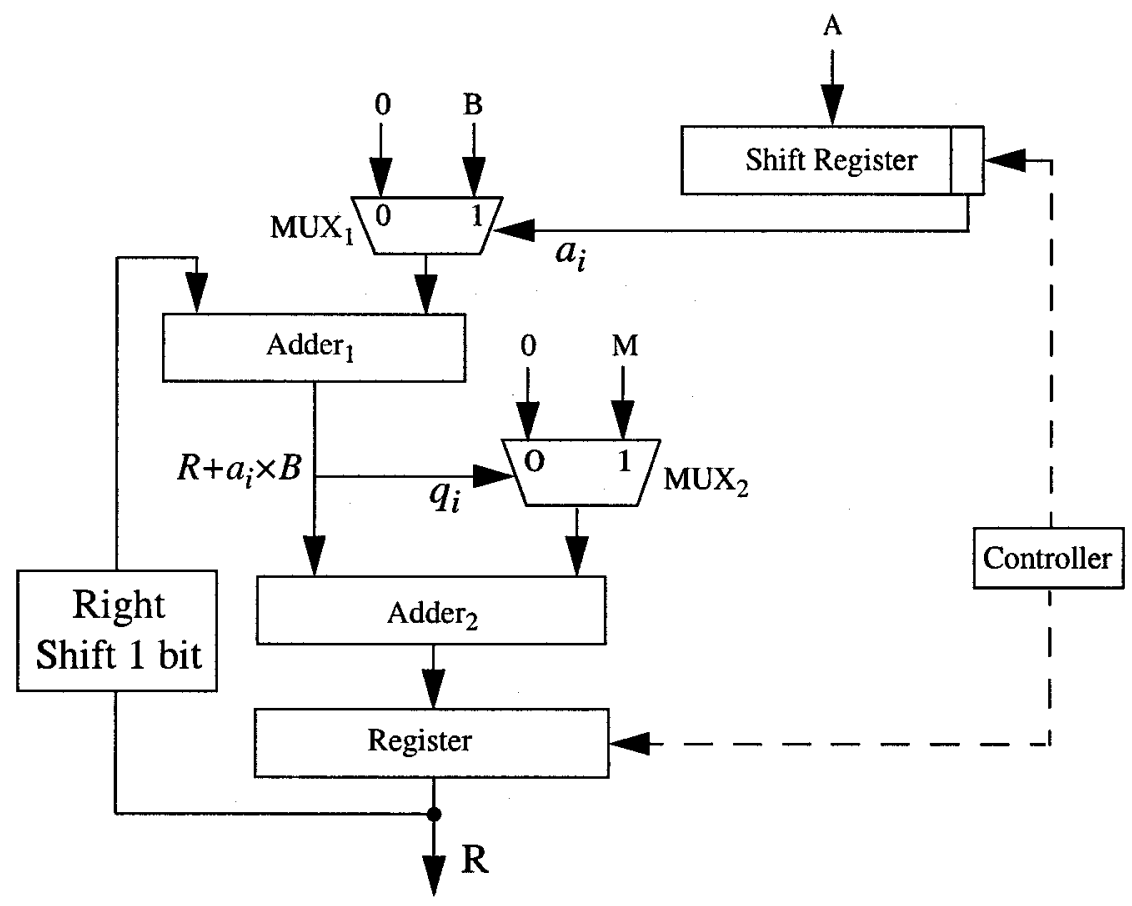

Figure 3.2 Simple hardware mapping of algorithm 3.1

Cells $M U X_{1}$ and $M U X_{2}$ are made up of $N$ two input multiplexers. The product $a_{i} \times B$ is implemented by $M U X_{1}$, then $A d d e r_{1}$ computes the first addition, $R=R+a_{i} \times B$ and then Adder $_{2}$ performs the second addition, $R=R+a_{i} \times B+M$ or $R=R+a_{i} \times B+O$. The augend of $\mathrm{Adder}_{2}$ is selected by $M U X_{2}$. "Right shift" is implemented by routing, requiring only minimal time to perform a division by two. Since the modulus $M$ appearing in the RSA calculation is always odd, the output of Adder $_{2}$ will always be even so that no precision loss will occur when this is divided by 2 . 
But unfortunately, this simple implementation is not practical because the delay of a 1024-bit ripple carry adder is unacceptably long. Modification of the basic adder is needed if it is to be useful in a large bit width multiplier.

\subsection{Design Considerations}

The goal of this design is to provide high speed with acceptable power consumption, goals that often conflict.

\subsubsection{Large Number Addition and Redundant Representations}

The key to a fast multiplier is a fast adder, and the conventional method of binary addition involving two addends and a carry at each stage is not fast enough for real-time RSA work.

The most significant bit (MSB) of the sum of two $\mathrm{N}$-bit numbers written in conventional form actually depends on all $2 N$ bits of the input data. In general then, each addition involves the propagation of carry information from the LSB position to the MSB - the entire width of the adder. This long carry propagation is one of the main challenges in the design of the modular multiplier. The logically-easiest way of dealing with the problem, that of lookahead carry, involves significant circuit complexity and concomitant high power consumption and large silicon areas. Other approaches, such as the carry-select adder and the condition-sum adder suffer similarly.

Significant performance improvements not accompanied by excessive power demands can be had if a fundamentally different addition method is used, one which involves the augmentation of the customary pair of operands with redundant data information.

The most well-known such approach is the carry-save adder. The basic idea is to reduce the sum of three binary numbers to the sum of two binary numbers without using carry propagation. The speed of operation will thus be constant and independent of word 
length. For this reason, the carry-save adder is the best choice when two large numbers are to be added. But the method will be a consumer of possibly scarce hardware resources, in that extra silicon area is required for the additional lengthy storage registers. Therefore carry save adders also will not be considered further.

\subsubsection{Pipelining}

The type of modular multiplication algorithm being considered here works by iteratively adding shifted versions of one of the operands. The speed of such a multiplier is limited by adder speed, and to achieve adequate performance it may be necessary to do multiple additions in parallel, using a pipelining technique. With appropriate design, the time penalty of carry propagation of long words can be made insignificant.

By way of example, a 4-bit pipelined ripple carry adder consisting of three 1-bit full adders and one 1-bit half adder is illustrated in Figure 3.3. The input data to its four one-bit stages are $a_{3}$ through $a_{0}$ and $b_{3}$ through $b_{0}$. The sum is presented at the outputs Sum $_{4}$ through Sum $_{0}$. Each stage has two output quantities, a sum bit and a carry-out (Cout) bit, which must be preserved from one clock to the next. D type flip-flops (DFFs) are used for this purpose.

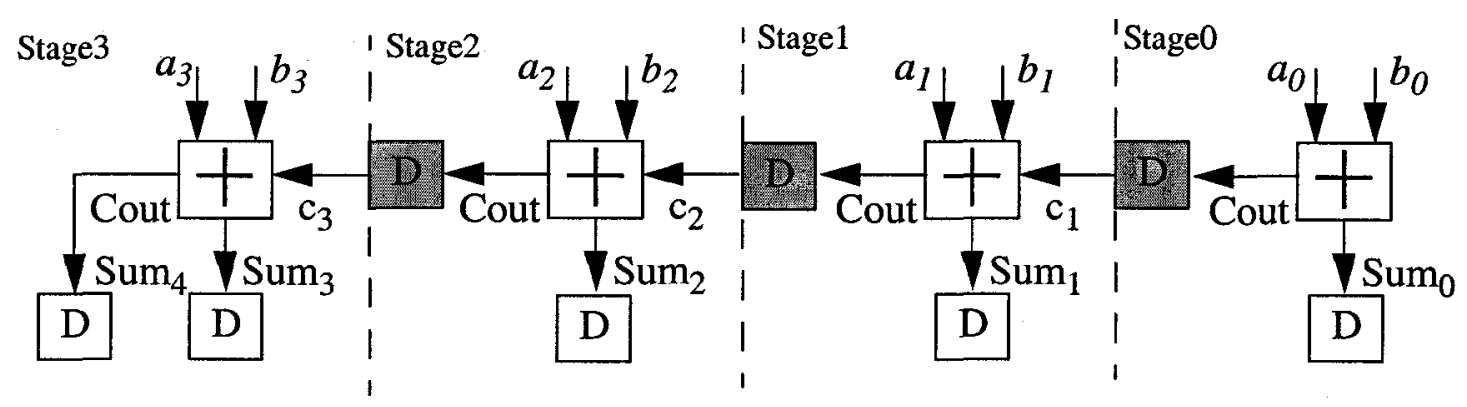

Figure 3.3 Pipelined 4-bit full adder

The DFFs also serve to isolate the stages from one another, permitting a $K$-stage pipeline to work on $K$ independent additions simultaneously. 
Table 3.1 illustrates the quantities appearing during the operation of a pipelined 4bit adder. We can see that at the fourth and fifth clock cycle, there are four instances processed simultaneously.

TABLE 3.1: Computing sequence of a pipelined 4-bit adder

\begin{tabular}{|c|c|c|c|c|}
\hline Clock Cycle & $\begin{array}{c}\text { Full adder3 } \\
\text { (Stage 3) }\end{array}$ & $\begin{array}{c}\text { Full adder2 } \\
\text { (Stage 2) }\end{array}$ & $\begin{array}{c}\text { Full adder1 } \\
\text { (Stage 1) }\end{array}$ & $\begin{array}{c}\text { Half adder0 } \\
\text { (Stage 0) }\end{array}$ \\
\hline \hline 1 & & & $a_{0}{ }^{(1)}+b_{0}{ }^{(1)}$ \\
\hline 2 & & $a_{2}{ }^{(1)}+b_{2}{ }^{(1)}+c_{2}{ }^{(1)}$ & $a_{1}{ }^{(2)}+b_{1}{ }^{(2)}+c_{1}{ }^{(2)}$ & $a_{0}{ }^{(3)}+b_{0}{ }^{(3)}$ \\
\hline 3 & $a_{3}{ }^{(1)}+b_{3}{ }^{(1)}+c_{3}{ }^{(1)}$ & $a_{2}{ }^{(2)}+b_{2}{ }^{(2)}+c_{2}{ }^{(2)}$ & $a_{1}{ }^{(3)}+b_{1}{ }^{(3)}+c_{1}{ }^{(3)}$ & $a_{0}{ }^{(4)}+b_{0}{ }^{(4)}$ \\
\hline 5 & $a_{3}{ }^{(2)}+b_{3}{ }^{(2)}+c_{3}{ }^{(2)}$ & $a_{2}{ }^{(3)}+b_{2}{ }^{(3)}+c_{2}{ }^{(3)}$ & $a_{1}{ }^{(4)}+b_{1}{ }^{(4)}+c_{1}{ }^{(4)}$ & $a_{0}{ }^{(5)}+b_{0}{ }^{(5)}+b_{0}{ }^{(2)}$ \\
\hline
\end{tabular}

\subsubsection{Circuit Speed}

The time required for a modular multiplication is determined by the product of two factors

(Addition time) * (Number of additions).

Both factors are investigated in this thesis. The time required for a single addition is reduced by careful critical path optimization (transistor level), and the number of iterations (additions) required is reduced by an architectural change, namely a radix increase from 2 to 4 .

\subsubsection{Choice of Logic Style}

The large word lengths (more than 1000 bits) of RSA operands greatly exceed the 16, 32 and 64-bits of general-purpose computers, and so efficient calculation requires hardware implementation. The power consumed by this hardware is an important matter, 
and low supply voltages provide the easiest route to low-power operation. The choice of logic style is therefore of great importance [30].

Conventional CMOS logic is selected in this design for the following reasons. First, the overall power dissipation of dynamic logic gates can be significantly higher than that of the static logic gates, although dynamic logic gates only consume dynamic power [29]. Second, dynamic logic gates have higher sensitivity to noise than static logic gates. Third, conventional CMOS logic is more amenable to voltage scaling than all the other static logic styles [29][30], making low-power design easiest. The final design can operate over a range of supply voltage from $1.0 \mathrm{~V}$ to $1.8 \mathrm{~V}(0.18 \mu \mathrm{m}$ CMOS $)$. Fourth, conventional CMOS logic is ratioless and the design is easy [30].

\subsubsection{Data dependency of Montgomery's Algorithm (Recursive)}

Montgomery's algorithm is a recursive process wherein the result at each step can be written as a function of the results of previous step(s). The main operations include quotient $\left(q_{i}\right)$ determination and product accumulation $\left(R^{(i)}=R^{(i-1)}+q_{i} \times M\right)$. These items are calculated in the sequence $q_{0}, R^{(0)}, q_{1}, R^{(1)}, q_{2}, R^{(2)}, \ldots$. In fact, $q_{i}$ can be computed from the least significant bit of $R^{(i-1)}$, i.e., $r_{0}^{(i-1)}$, so the sequence actually used is $q_{0}, r_{0}^{(0)}, q_{1}$, $r_{0}^{(1)}, q_{2}, r_{0}^{(2)} \ldots$

Since two items, $q_{i}$ and $R^{(i)}$ must be successive calculated in each iteration, two clock cycles are needed and the delay time between iterations is two clock cycles, i.e., there is one clock cycle gap between the appearances of $r_{0}{ }^{(i)}$ and $r_{0}{ }^{(i+1)}$. The cause of this is a data dependency between $r_{0}{ }^{(i)}$ and $r_{0}{ }^{(i+1)}$ [25]. In the following sections, to speed up the computation, we propose several techniques of solving this dependency problem and these will be examined in upcoming sections. 


\subsection{Radix-2 Architecture}

In this section, a radix-2 architecture is presented. The more-complicated but much faster radix-4 architecture is described in section 3.9.

\subsubsection{Modified Algorithm and Architecture}

After modification to the original architecture and algorithm, we propose the following algorithm and its implementation.

\section{Algorithm 3.2 (Radix-2)}

$\left\{1 . \mathrm{R}^{(-1)}=0 ; \mathrm{BM}=\mathrm{B}+\mathrm{M} ; / /\right.$ " $\mathrm{B}+\mathrm{M}$ " is computed in advance

2. For $\mathrm{i}=0$ To $\mathrm{N}-1$ \{

3. $q_{i}=r_{0}^{(i-1)} \oplus\left(a_{i} \cdot b_{0}\right)$

4. Case $q_{i}, a_{i}$

$4.1 \quad 0,0: \mathrm{R}^{(\mathrm{i})}=\left(\mathrm{R}^{(\mathrm{i}-1)}+0\right) / 2 ;$

$4.2 \quad 0,1: \mathrm{R}^{(\mathrm{i})}=\left(\mathrm{R}^{(\mathrm{i}-1)}+\mathrm{B}\right) / 2 ;$

$4.3 \quad 1,0: \mathrm{R}^{(\mathrm{i})}=\left(\mathrm{R}^{(\mathrm{i}-1)}+\mathrm{M}\right) / 2 ;$

$\left.4.4 \quad 1,1: \mathrm{R}^{(\mathrm{i})}=\left(\mathrm{R}^{(\mathrm{i}-1)}+\mathrm{BM}\right) / 2 ;\right\}$

5. Return $\mathrm{R}^{(\mathrm{N}-1)}$;

\section{\}}

In the original algorithm, for the worst case, two addition operations are required in each For loop: $R^{(i-1)}+a_{i} \times B$ and $R^{(i-1)}+M$. In this modified algorithm, the two additions are combined: $R^{(i)}=\left(R^{(i-1)}+B M\right)$ where $B M$ can be calculated in advance. Here we suppose the input data, $B$ and $M$ are stable during the whole computation. This combination is implemented by using MUX4-1 cells. 
Figure 3.4 shows the hardware version of the modified algorithm. Thick lines represent multi-bit data lines and thin lines represent 1 -bit data lines unless indicated otherwise by a number. The dashed box of the figure encloses a multiplier 'bit-slice', a number of which can be adjoined to assemble in assembling a modular multiplier of whatever width is needed. Its architectural details will be presented in section 3.7.4. The pre-compu-

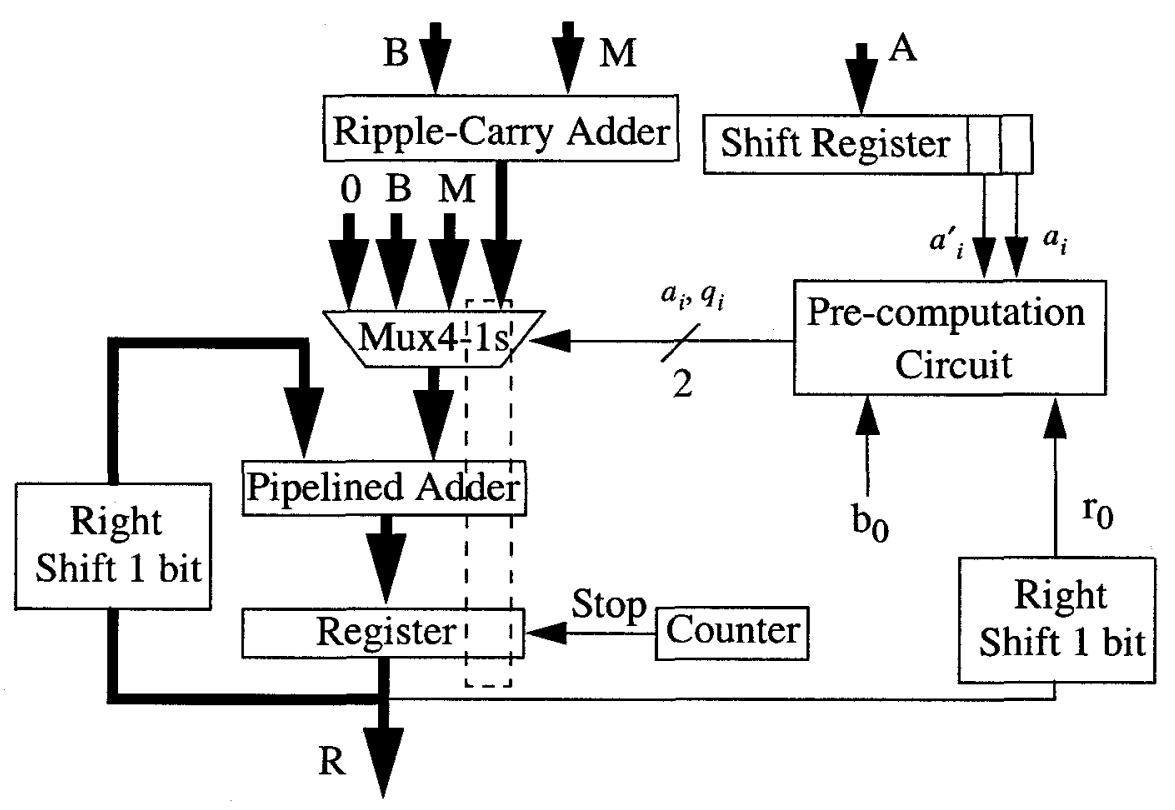

Figure 3.4 Modified architecture

tation circuit performs step 3 of algorithm 3.2 and will be presented in section 3.7.7.

\subsubsection{Data Dependency and Signal Flow Graphs}

A Dependence Graph (DG) pictorially describes the interrelationships of the data involved in a calculation [31].

If a DG is to be fully exploited, the algorithm must be written so that there is a oneto-one correspondence between it and its graph. In particular all data items must appear explicitly. Making these changes to Algorithm 3.2 results in

$\left\{1 . \mathrm{R}^{(-1)}=0 ; \mathrm{BM}=\mathrm{B}+\mathrm{M}\right.$

2. For i=0 To N-1 \{ 
3. $q_{i}=r_{0}^{(i-1)} \oplus\left(a_{i} \times b_{0}\right)$;

4. $\quad$ For $\mathrm{j}=0$ To $\mathrm{N}$

\section{$4.1\left\{\right.$ Case $q_{i}, a_{i}$ :}

$$
0,0:\left\{\mathrm{r}_{\mathrm{j}}^{(\mathrm{i})}=\operatorname{Sum}\left(\mathrm{r}_{\mathrm{j}+1}{ }^{(\mathrm{i}-1)}+\mathrm{c}_{\mathrm{j}}^{(\mathrm{i}-1)}+0\right) ; \mathrm{c}_{\mathrm{j}}^{(\mathrm{i})}=\operatorname{Carry}\left(\mathrm{r}_{\mathrm{j}+1}{ }^{(\mathrm{i}-1)}+\mathrm{c}_{\mathrm{j}}^{(\mathrm{i}-1)}+0\right)\right\}
$$$$
0,1:\left\{\mathrm{r}_{\mathrm{j}}^{(\mathrm{i})}=\operatorname{Sum}\left(\mathrm{r}_{\mathrm{j}+1}{ }^{(\mathrm{i}-1)}+\mathrm{c}_{\mathrm{j}}^{(\mathrm{i}-1)}+\mathrm{b}_{\mathrm{j}}\right) ; \mathrm{c}_{\mathrm{j}}^{(\mathrm{i})}=\operatorname{Carry}\left(\mathrm{r}_{\mathrm{j}+1}{ }^{(\mathrm{i}-1)}+\mathrm{c}_{\mathrm{j}}^{(\mathrm{i}-1)}+\mathrm{b}_{\mathrm{j}}\right)\right\}
$$

$$
1, O:\left\{\mathrm{r}_{\mathrm{j}}^{(\mathrm{i})}=\operatorname{Sum}\left(\mathrm{r}_{\mathrm{j}+1}^{(\mathrm{i}-1)}+\mathrm{c}_{\mathrm{j}}^{(\mathrm{i}-1)}+\mathrm{m}_{\mathrm{j}}\right) ; \mathrm{c}_{\mathrm{j}}^{(\mathrm{i})}=\operatorname{Carry}\left(\mathrm{r}_{\mathrm{j}+1}{ }^{(\mathrm{i}-1)}+\mathrm{c}_{\mathrm{j}}^{(\mathrm{i}-1)}+\mathrm{m}_{\mathrm{j}}\right)\right\}
$$

\section{Return $\mathrm{R}^{(\mathrm{N}-1)}$;}

\section{\}}

In the above algorithm, the variable $i$ is the iteration index and $j$ is the bit number of the multiplier; that is, an $N$-bit multiplier was assembled from $N+11$-bit cells connected serially. It is observed that a cell mainly consists of one 1-bit full adder and a MUX4-1.

The two-dimensional DG corresponding to this algorithm is shown in Figure 3.5, where $N=4$ has been assumed. The DG consists of $20(4 \times 5)$ basic cells. The input data, $B$, $M$ and $B M$, enter the DG from the top and $A$ from the right. Each cell has one 1 -bit full adder to perform its 1 -bit addition. The three pieces of data accepted as input by the 1 -bit full adder, namely addend, carry and augend, are $r_{j+1}{ }^{(i-1)}, c_{j}^{(i-1)}$ and the output from MUX4-1 respectively. 
None of the data bits $q_{i}, b_{j}, m_{j}, b m_{j}$, and $a_{i}$ are altered as they 'move' through the graph. After $N$ iterations, the final results, $r_{j}$, have appeared at the bottom of the array.

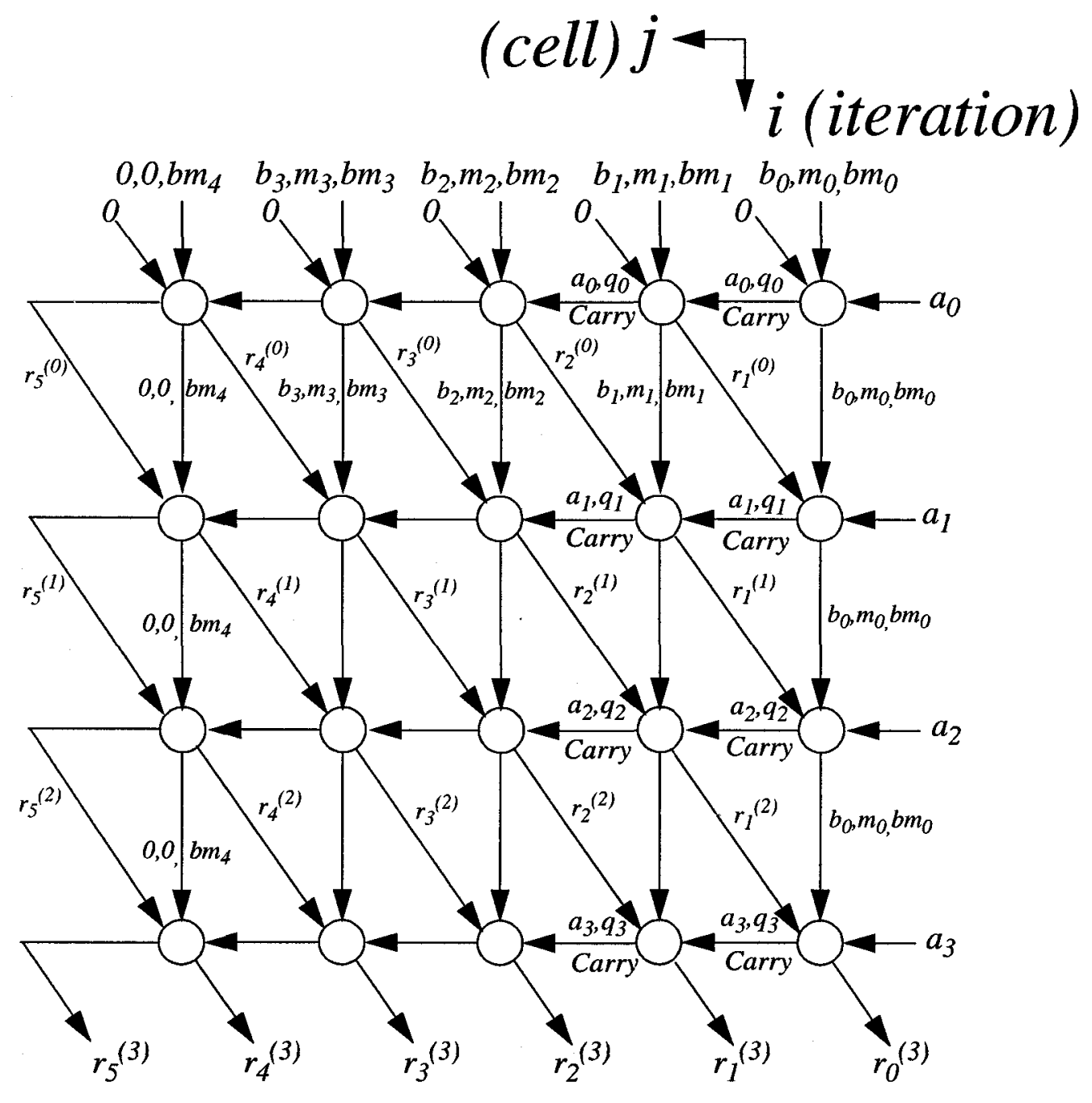

Figure 3.5 DG of modified algorithm

From the data Dependence Graph, we can use the projection method [31] to produce a signal flow graph (SFG), which is more closely analogous to the hardware than is the DG. 
An SFG can be derived from a DG by projecting the two dimensional graph on to one dimensional graph. In Figure 3.6, DG was divided into five parts and then projected in the vertical direction [31].
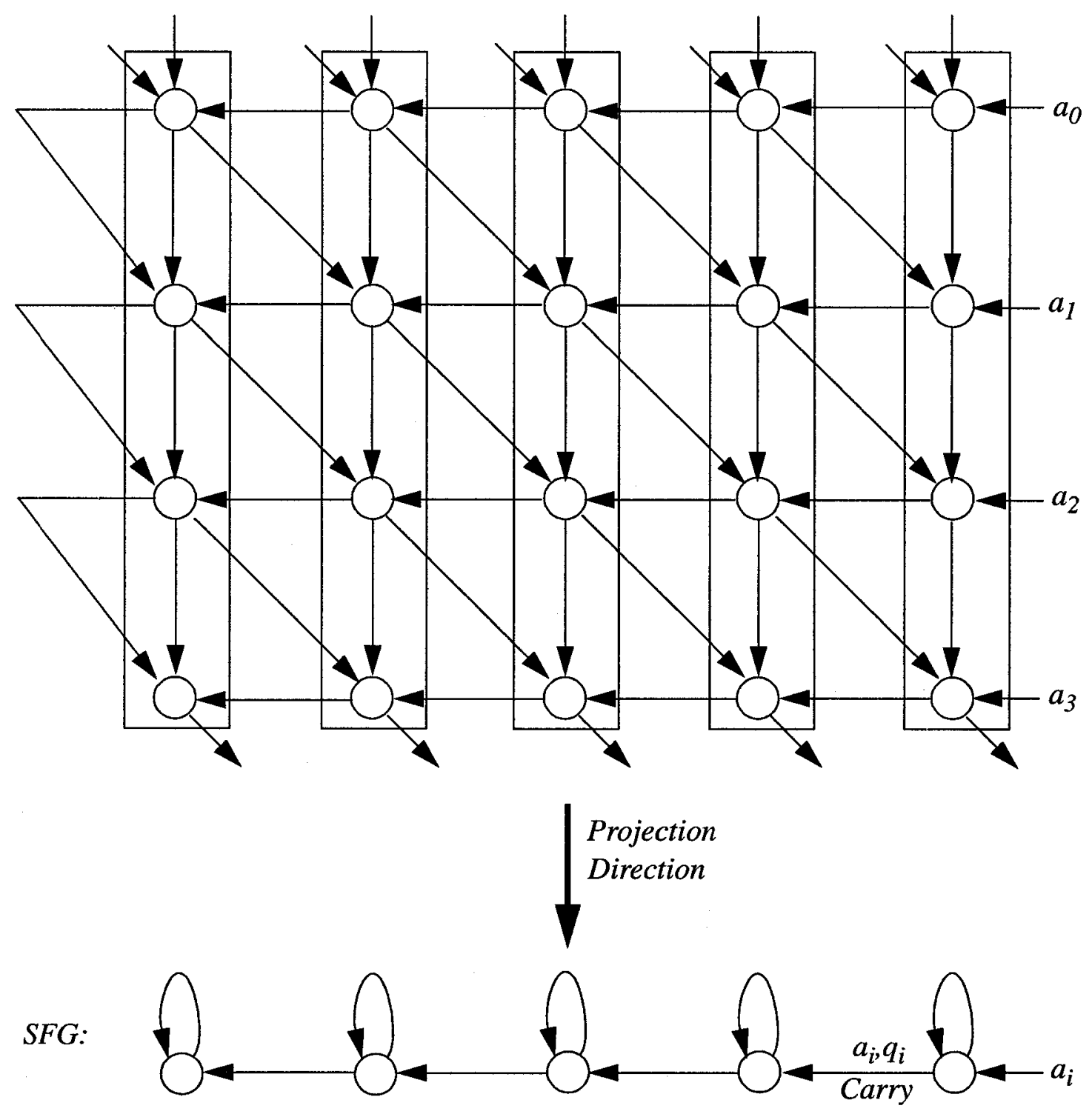

Figure 3.6 Projecting a DG to an SFG

The labels of the DG are the same as those in the previous figure.

If one applies a schedule line (timing sequence) to the SFG, a systolic array is obtained. 


\subsubsection{Clock Signals}

A clock signal determines when the sequential components save the output of the combinational circuits. The clocking methodologies significantly affect the functionality, speed, and power of a circuit [29].

\subsubsection{Single Phase Clock Signal}

The simplest clock signal is the single-phase one. In this design, all gates are static types. Since it is only the DFFs that require clocking, a single phase clock will be adequate.

\subsubsection{Utilization of Two Edges of a Clock Signal}

Usually, a sequential circuit uses DFFs that operate on just one of the clock edges, the positive one or the negative one. The present design however uses both positive and negative edge triggered DFFs.

\subsubsection{Unsuitability of Double Edge Triggered DFFs}

Double edge triggered DFFs can utilize both rising and falling edges of a clock signal. However, in this design, the standard double edge DFF is not suitable because of a data dependency occurring in the recursive calculation of $R$ : Figure 3.7 shows that $r_{2}{ }^{(i)}$ must be stable during the calculation of $r_{1}{ }^{(i+1)}$. Therefore $r_{1}{ }^{(i+1)}$ and $r_{2}{ }^{(i)}$ can not be triggered on the same edge of the clock signal.

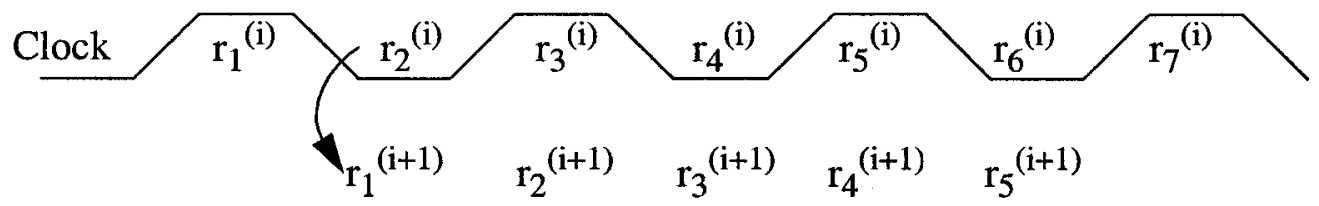

Figure 3.7 Data dependency of intermediate result 


\subsubsection{Pipeline approach}

The challenges of using Montgomery modular multiplication in cryptographic applications take the form of wide data words and lengthy iteration times. Each stage of the iteration must have available the result of the previous iteration. The data dependencies of Montgomery's algorithm imply the sequence of calculation shown in Figure 3.8.

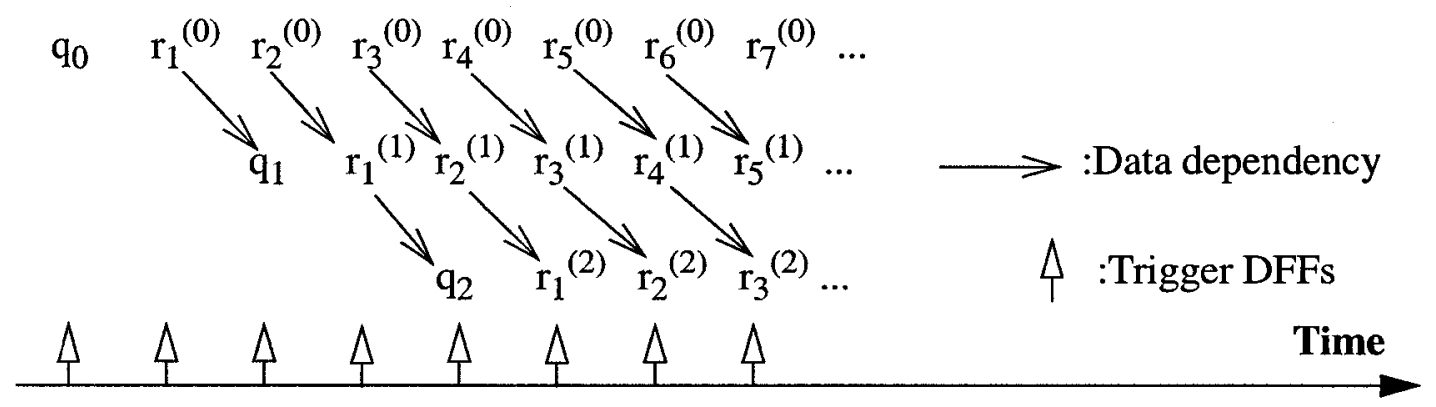

Figure 3.8 Timing of $r_{j}^{(k)}$ and $q_{k}$

Some data dependencies between two successive iterations are also shown in this figure.

Pipelining techniques may be used to reduce the total computation time of a modular multiplication. The clocking scheme that is used depends on the details of the pipeline architecture. Figure 3.9 shows three possible approaches for pipelined 6-bit FAs. All these adders use the Sum results, i.e., $r_{2}$ through $r_{7}$, as their left augend respectively, just like the 
Pipelined Adder in Figure 3.4 on page 34. In Figure 3.9 (a) and (b), one 1-bit FA is used for each pipeline stage, while in Figure 3.9 (c) a 2-bit FA is employed per stage.

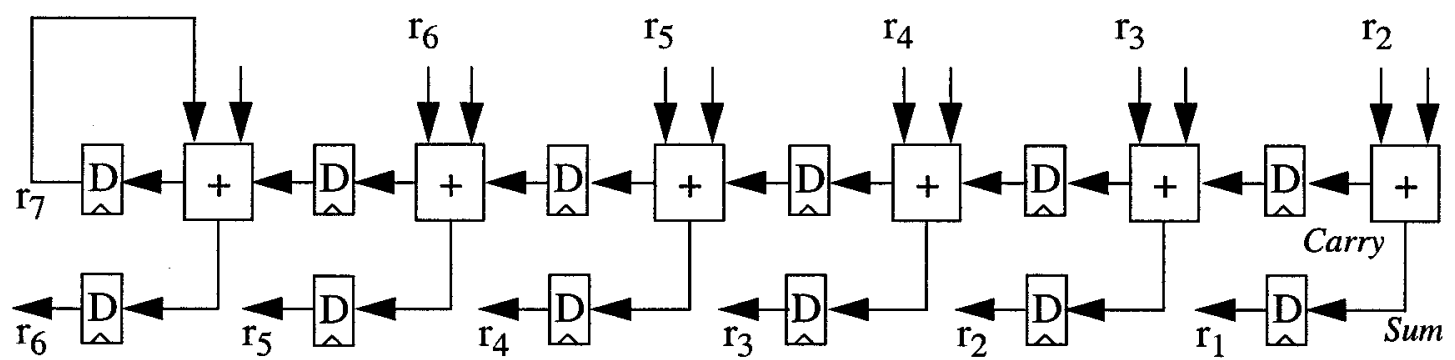

(a) 1 bit per stage with one type of D flip flops

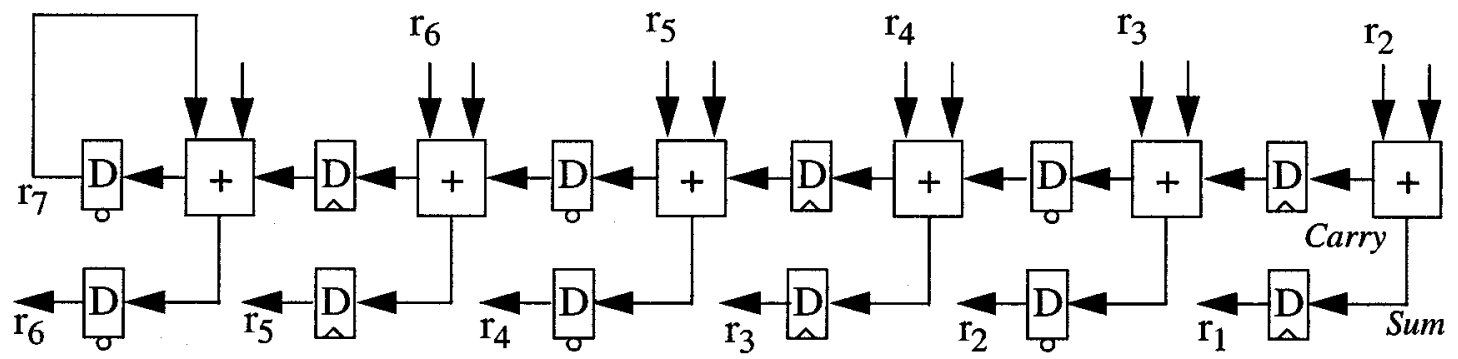

(b) 1 bit per stage with two types of D flip flops

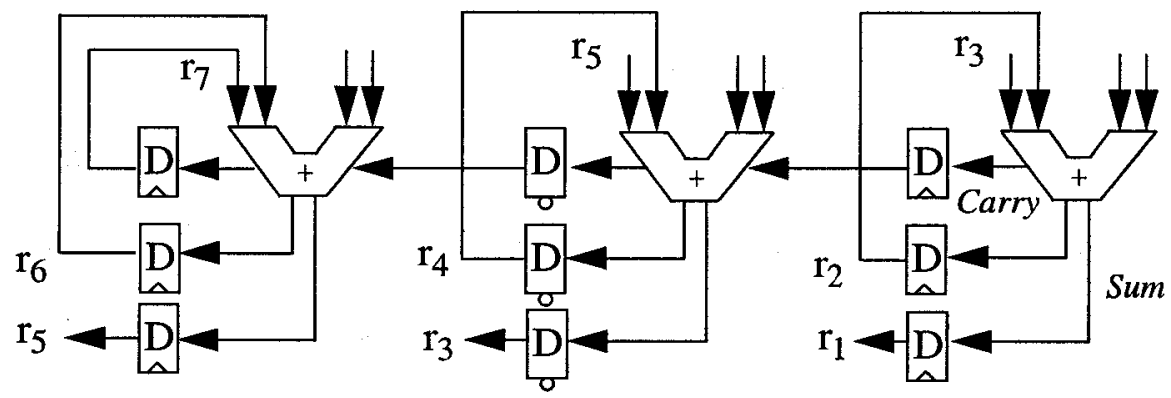

(c) 2 bit per stage with two types of $\mathrm{D}$ flip flops

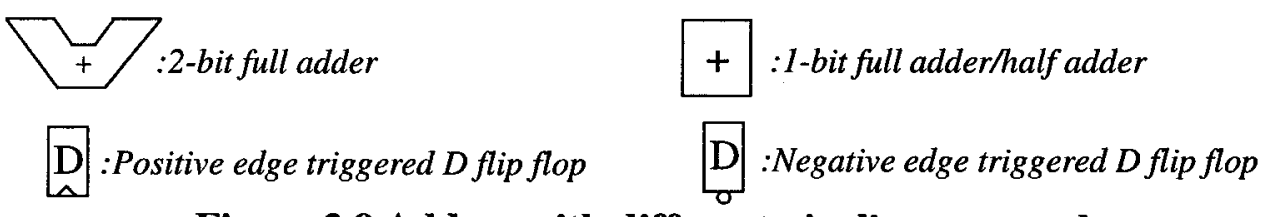

Figure 3.9 Adders with different pipeline approaches

(Right addend of each adder is not shown)

Three clocking approaches for the hardware arrangement shown in Figure 3.9 are illustrated in Figure 3.10. 


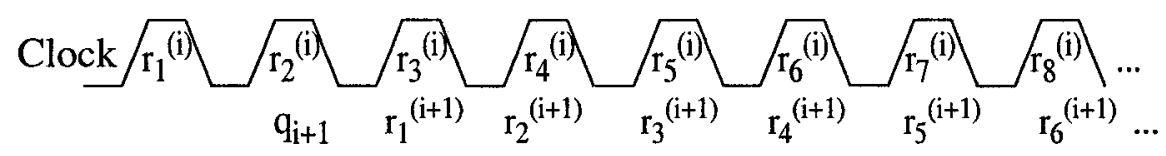

(a) Clocking approach a - Single edge 1-bit

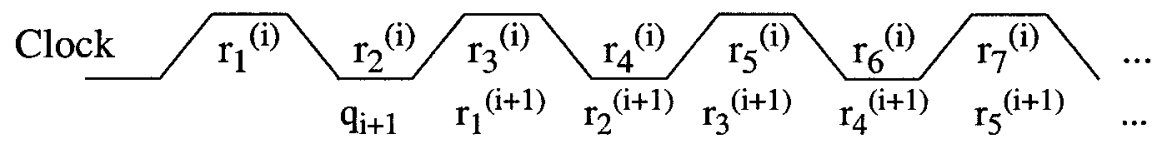

(b) Clocking approach b - Double edge 1-bit

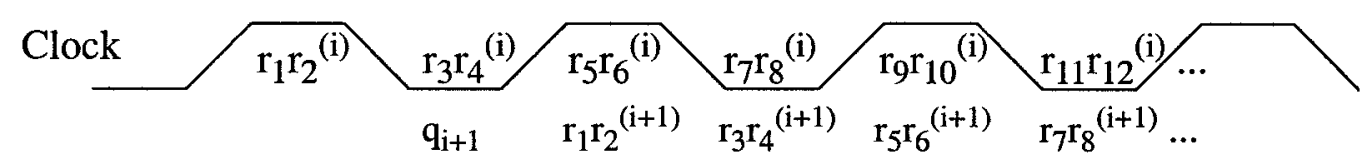

(c) Clocking approach $\mathrm{c}$ - Double edge 2-bit

Figure 3.10 Three clocking approaches for pipeline stages

The simplest one is shown in Figure 3.10 (a). Its corresponding hardware configuration is illustrated in Figure 3.9 (a). The data dependency between $r_{j}^{(i)}$ and $r_{j-1}{ }^{(i+1)}$ requires two clock cycles between adjacent iterations instead of one (see $r_{2}{ }^{(i)}$ and $r_{2}{ }^{(i+1)}$ ). This characteristic is called the "one-clock-cycle gap" [25]. Some designs overcame the problem by interleaving two independent modular multiplications in one multiplier to compute exponentiation via the right-to-left modular exponentiation algorithm. However, as we have discussed in Chapter 2 (See "Security Analysis of Exponentiation Algorithms" on page 22), the right-to-left method is not safe from certain side-channel attacks. Because of this, interleaving methods will not be discussed any further in this thesis.

For an $N$-bit multiplication, the latency of the first pipeline approach (Figure 3.10 (a)) is $2 N+N$, i.e., $3 N$ clock cycles. We are proposing a different pipeline approach employing both edges of the clock signal, shown in Figure 3.10 (b). The one-clock-cycle gap problem in Figure 3.10 (a) is removed since the $r_{j}^{(i)}$ are ready when the $r_{j-1}{ }^{(i+1)}$ are to be calculated. The latency in this case is reduced to $N+N / 2$, i.e., $1.5 N$ clock cycles.

For a sequential circuit whose cycle time satisfies 


$$
T>T_{p, c o m b}+T_{c q}+T_{\text {setup }}
$$

with $T_{p, c o m b}$ the longest delay of any combinational circuitry, $T_{c q}$ the propagation delay (usually called clock-to- $Q$ delay) of a flip-flop, and its setup time, $T_{\text {setup }}$. A clocking approach like that of Figure 3.10 (b) is used, where a nonstandard dual edge-triggered system is used. Furthermore, by combining two 1-bit FAs we can derive the third pipeline approach in Figure 3.9 (c) (Its clocking approach is shown in Figure 3.10 (c)). The clock period does not have to be doubled compared with that of clocking approach (b). In this case, the latency is further reduced to $N+N / 4$, or $1.25 N$ clock cycles. From the power consumption point of view, the pipeline approach of Figure 3.9 (c) requires the least hardware and switching times of the three to perform one modular multiplication.

Figure 3.11 gives examples of an 8-bit modular multiplication based on these pipeline approaches.

In each diagram, one dimension is "Time-Unit/Clock Cycle" and the other "Iteration Number", with "Time-Unit" being the generalized time. All examples require a total of eight iterations, due to the 8-bit word size. Figure 3.11(a) shows pipeline approach (a) where a 1-bit addition per clock cycle is done, and 22 cycles are needed for the whole multiplication. In Figure 3.11 (b) and (c), the required number of clock cycles are 11 and 9 , respectively. If we define Time-Unit to be one generalized interval of time, we will have $T_{a}=$ Time-Unit. Approach (b) uses two types of edge triggered flip flops and performs a 2 bit addition in one cycle. The clock cycle time is doubled $\left(T_{b}=2 T_{a}\right)$, but the computation time is the same as that of (a). Approach (c), where two 1-bit FAs are cascaded and two types of edge triggered flip flops are used, the cycle time is doubled once again $\left(T_{c}=4 T_{a}\right)$ and the computation time is much longer than that of (b). Therefore, as shown, the pipeline approach (c) is an inferior architecture even though it performs a 4-bit addition in each cycle. But this architecture is promising in that it does allow a 4-bit data dependency and results in less hardware. The problem is its long clock cycle which results from two 
cascaded 2-bit FA delays. We optimize the 2-bit FA to shorten the long clock cycle from 4 to 2.5 Time-Units. The details of optimizing the 2-bit adder cascade are illustrated in Figure 3.24 and section 4.1.2. Figure 3.11 (d) shows the example of the optimization. It requires $22.5(2.5 \times 9)$ Time-Units to finish one 8-bit multiplication; this is only $2.27 \%$ longer than that required in approach (a) or (b). The transistor count of the speed-optimized 2-bit FA increased from 56 (unoptimized) to 70 .

Figure 3.12 shows the hardware complexity of each pipeline approach.

We estimated the specifications of cycle time, latency, multiplication time and hardware cost of the three pipeline approaches of Figure 3.12; the results are summarized in Table 3.2. Switching times in Table 3.2 are those involved in triggering sequential components when performing a single $N$-bit modular multiplication. All data is relevant to $0.18 \mu \mathrm{m}$ CMOS technology. Specifications of approach (a) and (b) were estimated from the design details of the full custom cells. Specification of optimized approach (c) is obtained by extrapolating the post-layout simulation results of a 32-bit multiplier. From Table 3.2, we can see that approach (c) has the lowest latency, the smallest switching times, and uses the least hardware. It therefore consumes less power than the other two. The shorter the latency, the less the overall switching times, and therefore, the lower the power consumption (dynamic power). 


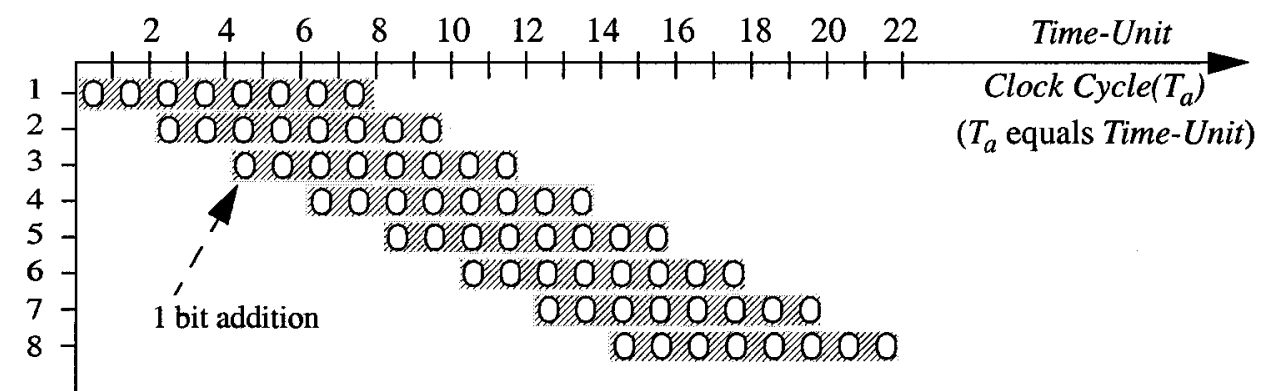

Iteration number $\quad$ (a) Example of pipeline approach (a)

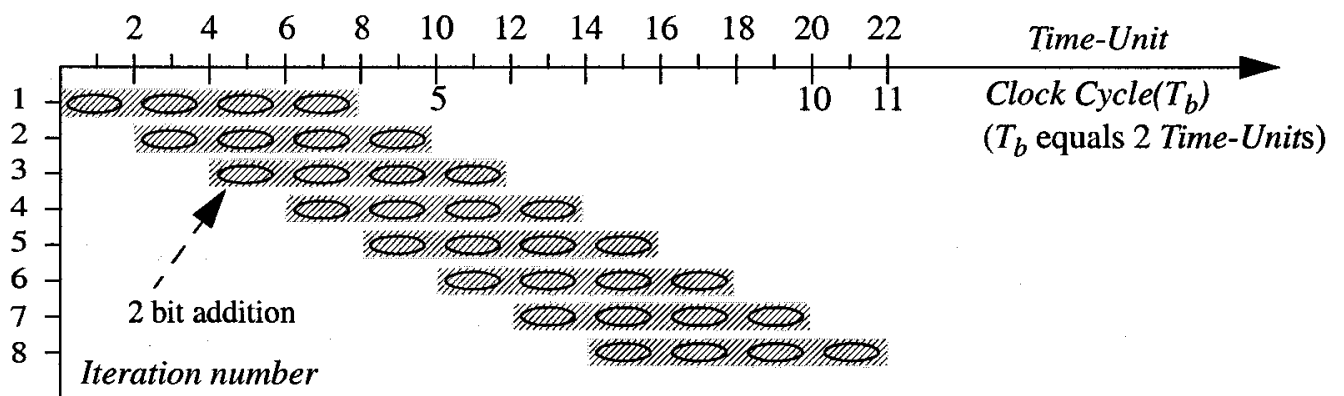

(b) Example of pipeline approach (b)

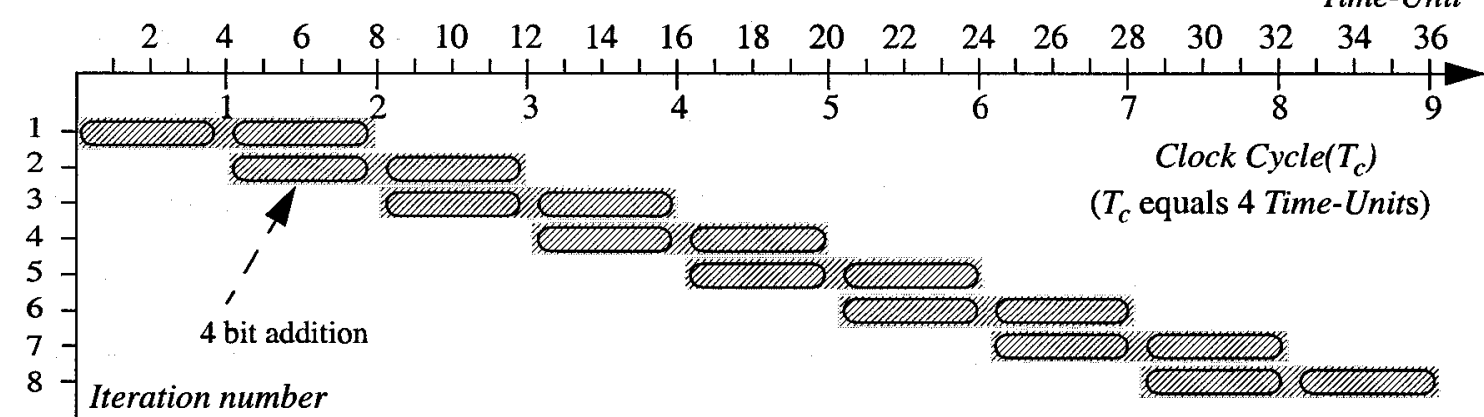

(c) Example of pipeline approach (c)

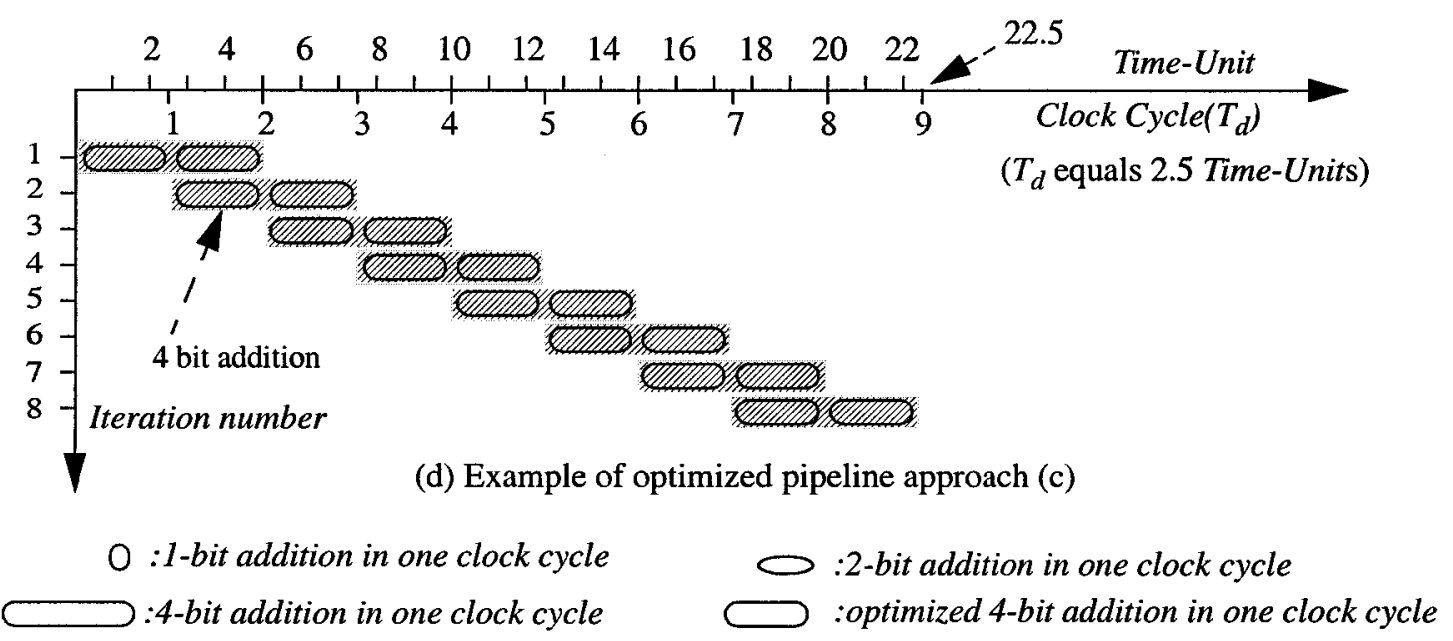

Figure 3.11 Examples of three pipeline approaches 


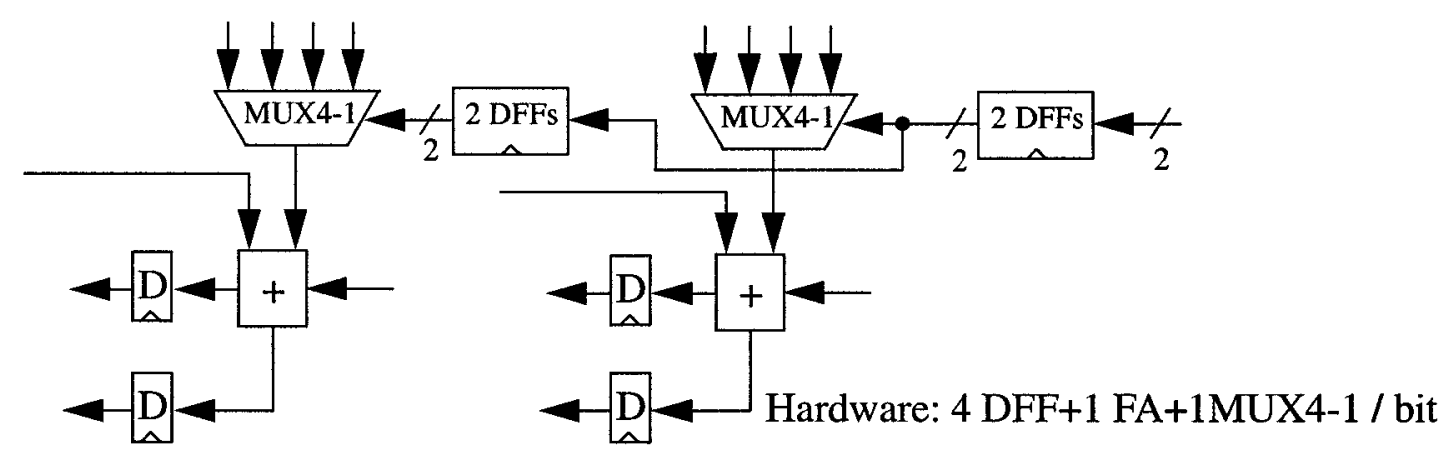

(a) Hardware cost of pipeline approach a

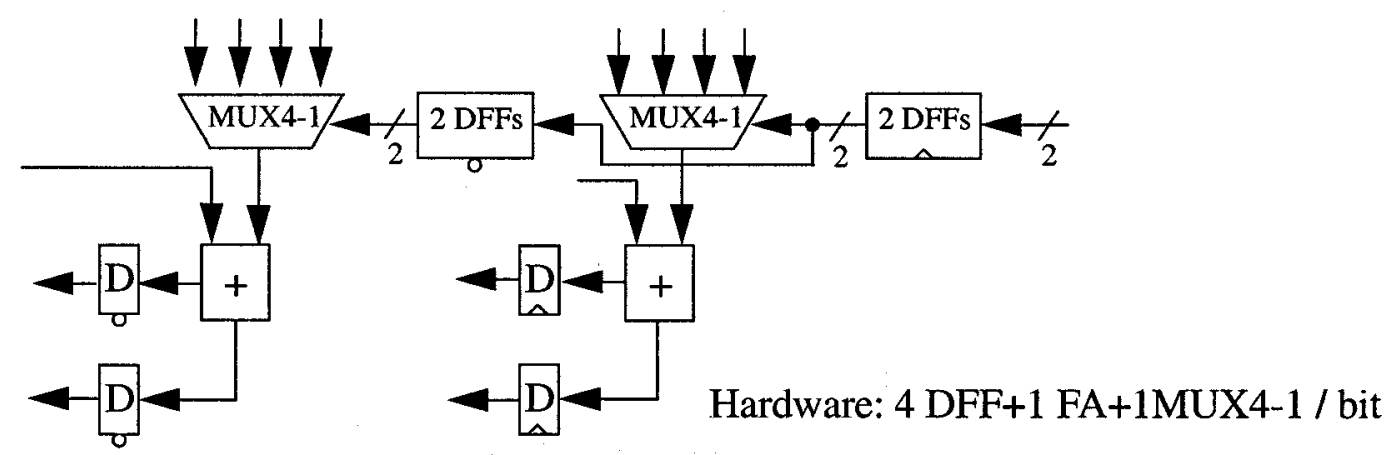

(b) Hardware cost of pipeline approach b

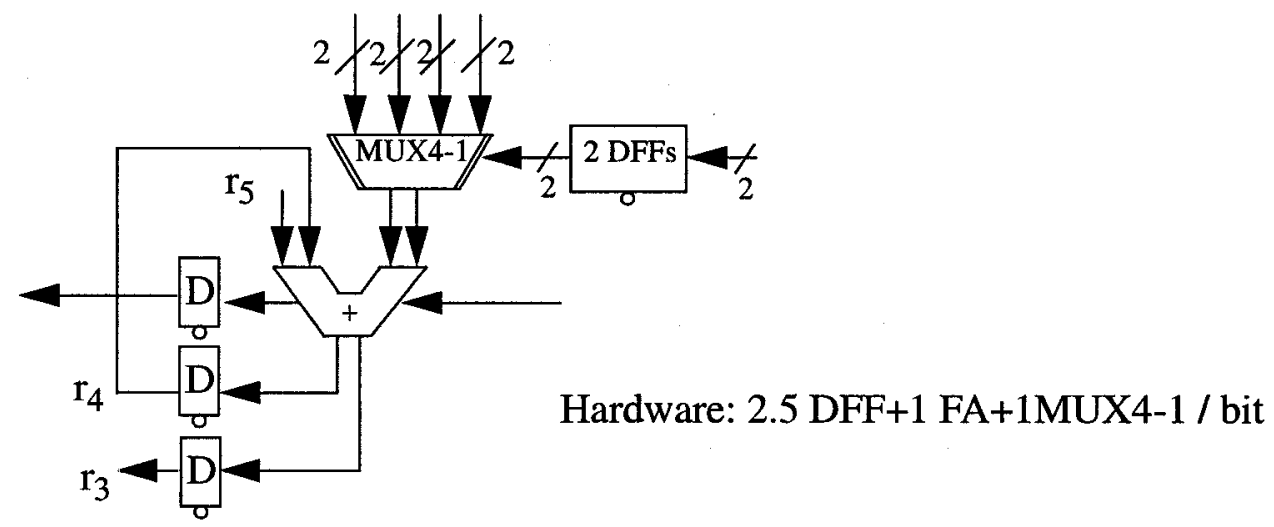

(c) Hardware cost of pipeline approach c

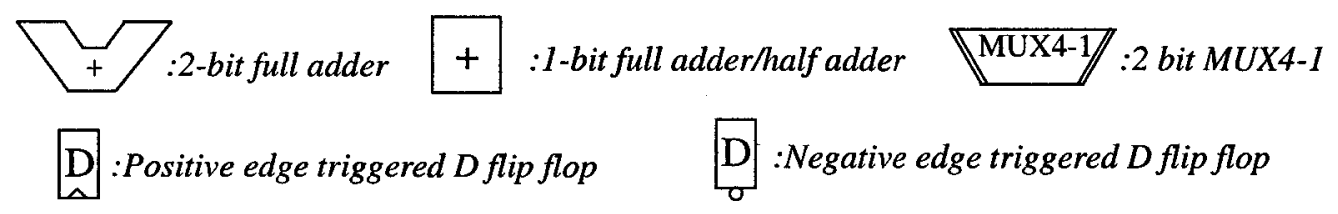

Figure 3.12 Hardware arrangement/complexity of each pipeline approach

Advantages of this clocking scheme: 
1. Lower power consumption.

2. Fewer clock cycles per modular multiplication.

3. Less demand on hardware resources.

TABLE 3.2: Comparison of the three pipeline approaches

\begin{tabular}{|l|l|c|c|c|c|}
\hline Approach & $\begin{array}{l}\text { Cycle } \\
\text { Time (ns) }\end{array}$ & Latency & $\begin{array}{c}\text { Multiplication } \\
\text { time (ns) }\end{array}$ & $\begin{array}{c}\text { Switching } \\
\text { times }\end{array}$ & Hardware/bit ${ }^{1}$ \\
\hline \hline (a) & $0.4^{2}$ & $3 N$ & $1.2 N$ & $3 N$ & $1 \mathrm{~A}_{\mathrm{FA}}+4 \mathrm{~A}_{\mathrm{DFF}}+1 \mathrm{~A}_{\mathrm{MUX} 4-1}$ \\
\hline (b) & $0.8^{3}$ & $1.5 N$ & $1.2 N$ & $3 N$ & $1 \mathrm{~A}_{\mathrm{FA}}+4 \mathrm{~A}_{\mathrm{DFF}}+1 \mathrm{~A}_{\mathrm{MUX} 4-1}$ \\
\hline $\begin{array}{l}\text { optimized } \\
\text { (c) }\end{array}$ & $1.0^{4}$ & $1.25 N$ & $1.25 N$ & $2.5 N$ & $1 \mathrm{~A}_{\mathrm{FA}}+2.5 \mathrm{~A}_{\mathrm{DFF}}+1 \mathrm{~A}_{\mathrm{MUX} 4-1}$ \\
\hline
\end{tabular}

1. $\mathrm{A}_{\mathrm{FA}}$ : Area of a 1-bit full adder; $\mathrm{A}_{\mathrm{DFF}}$ : Area of a $\mathrm{D}$ flip flop;

$A_{\text {MUX4-1: }}$ Area of a MUX4-1.

2. Critical path: $200 \mathrm{ps}+200 \mathrm{ps}=400 \mathrm{ps}\left(\mathrm{T}_{\mathrm{FA} 1}+\mathrm{T}_{\mathrm{DFF}}\right)$.

3. Critical path: $400 \mathrm{ps}+400 \mathrm{ps}=800 \mathrm{ps}\left(2 * \mathrm{~T}_{\mathrm{FA} 1}+2 \mathrm{~T}_{\mathrm{DFF}}\right)$.

4. Critical path: $600 \mathrm{ps}+400 \mathrm{ps}=1 \mathrm{~ns}\left(2 * \mathrm{~T}_{\mathrm{FA} 2}+2 \mathrm{~T}_{\mathrm{DFF}}\right)$.

$\mathrm{T}_{\mathrm{FA} 1}$ : Delay time of a 1-bit full adder;

$\mathrm{T}_{\mathrm{FA} 2}$ : Delay time of a 2-bit full adder;

$T_{\mathrm{DFF}}$ : Delay time of a $\mathrm{D}$ flip flop.

A minor disadvantage of clocking scheme (c) is that its multiplication time is longer than (b)'s by $4.2 \%$. In addition, the duty cycle should be $50 \%$ because two types of edge triggered $\mathrm{D}$ flip flops are used. The advantages of the scheme easily overwhelm these minor deficiencies.

\subsubsection{The Standard Bit Slice}

An entire multiplier is assembled from three types of slice elements, namely the standard, least and most significant bit slices, arranged as a series of standard slices terminated on either end by a least and a most significant slice. The standard slice must be used in pairs because of DFF triggering differences. The standard bit slice is outlined by the dashed box of Figure 3.4 on page 34, and is the one drawn in Figure 3.13. $b_{j+1} b_{j}, m_{j+1} m_{j}$, and $b m_{j+1} b m_{j}$ are corresponding bit pairs of $B, M$ and $B M$ respectively. The function of 
signal "Stop" is to enable or disable the DFFs which store the results of the 2-bit full adder. "Select" is a two-bit signal $\left(a_{i}, q_{i}\right)$ used to choose the correct addend from MUX4-1. The standard bit slice circuit has three main tasks:

1. Prepare the correct addend for the 2-bit FA;

2. Perform the addition; and

3. Propagate the data and control signals, such as Carry, Stop and Select, to neighboring cells.

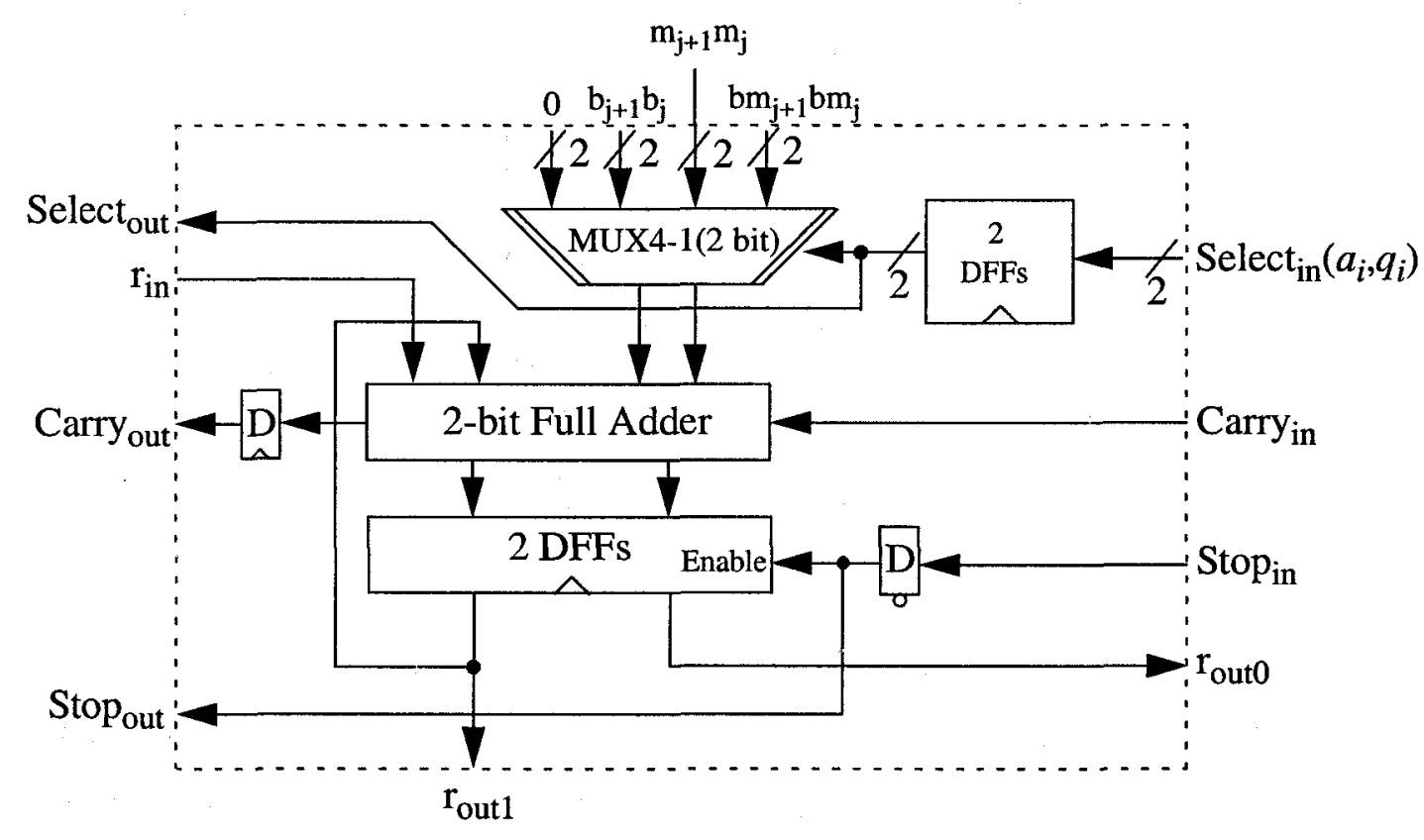

Figure 3.13 Standard bit slice circuit

There are two types of standard bit slice circuits: one with positive edge triggered D flip flops and one with negative edge triggered D flip flops. These are arranged in an alternating fashion - each positive type has two negative type cells as its two neighbors and vice versa. 


\subsubsection{Least Significant Bit Slice}

The least significant bit slice is arranged at the right boundary of the entire multiplier. It has one task: computing $r_{l}{ }^{(i)}$. The least significant bit slice circuit shown in Figure 3.14 is different from the standard bit slice. First, it does not use a MUX4-1 to select its addend, but rather a specialized circuit (Figure 3.16) for this purpose. Second, it

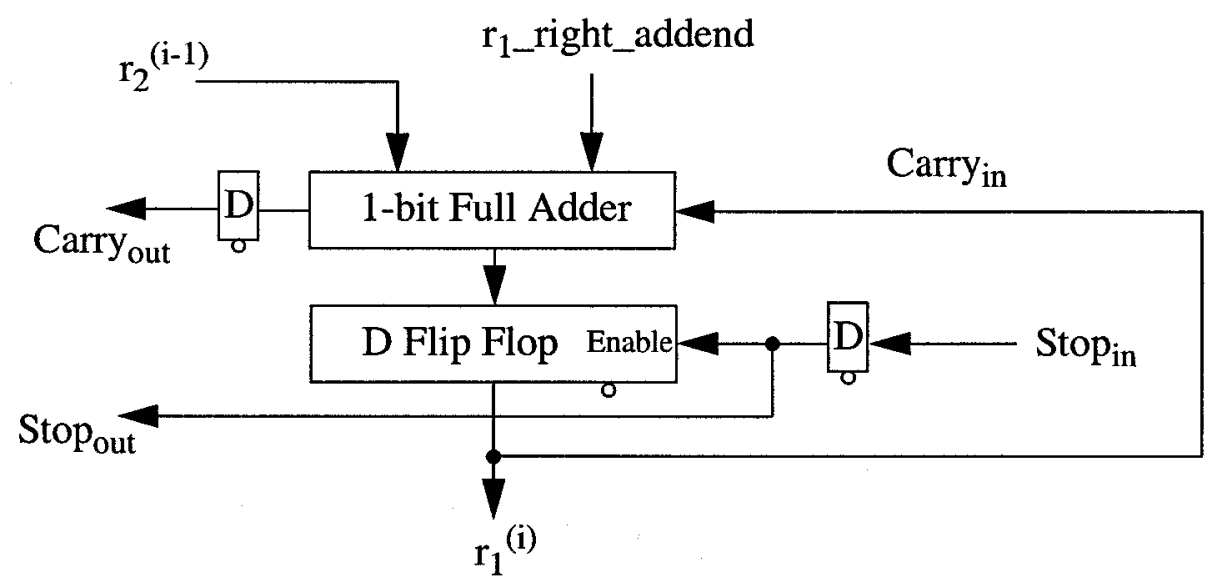

Figure 3.14 Least significant bit slice circuit

does not include a half adder (The eliminated half adder is shown in Figure 3.15) because the least significant bit of the result, $r_{0}{ }^{(i)}$, is known always to be zero. In addition, the carry signal from $r_{0}{ }^{(i+1)}$ is the same as $r_{1}{ }^{(i)}$ because $r_{1}{ }^{(i)}$ is the left augend of the 1-bit half adder for $r_{0}{ }^{(i+1)}$ and the right addend of the half adder will be the same as left augend to guarantee an even sum. Finally, the equality of the left augend and the right addend means 
that $\operatorname{Carry}_{0}{ }^{(i+1)}$ will be the same as the left augend. A direct connection of Carry ${ }_{\text {in }}$ to $r_{1}{ }^{(i)}$ (Figure 3.14) gets the job done.

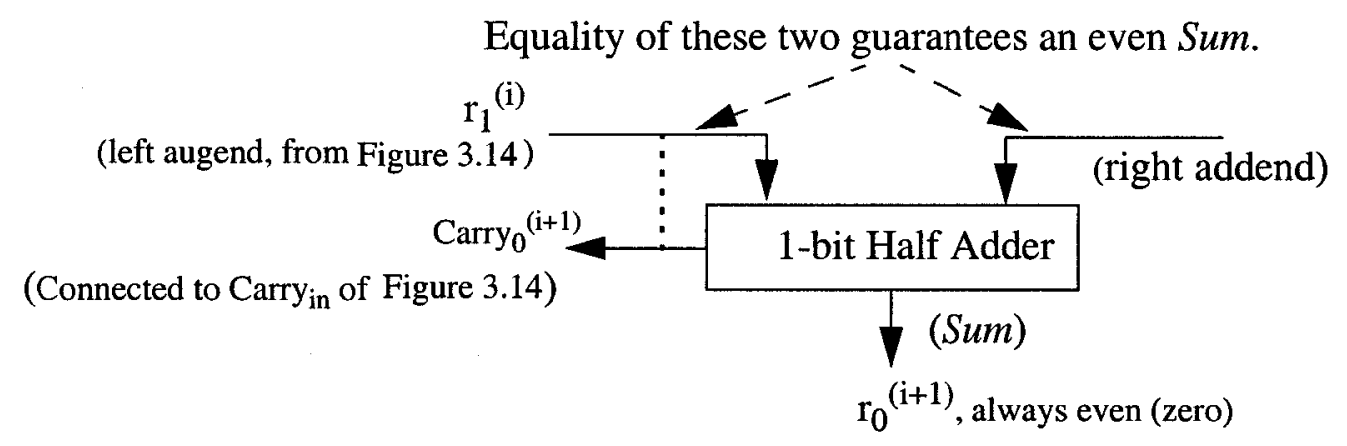

\section{Figure 3.15 Eliminated circuit for $\mathbf{r}_{\mathbf{0}}$}

The next question is one of how the right addend, $r_{1-}$ right_addend, for the 1-bit full adder in Figure 3.14 is obtained. Four possibilities for this number are $0, m_{1}, b_{1}$, and $b m_{1}$. Only one is selected as an addend for the 1-bit full adder. The selection signals are $a_{i}{ }_{i}$ and $q_{i}$ (refer to Figure 3.4 on page 34 and $a_{i}{ }^{\prime}$ comes from a shift register containing $A$, see Figure 3.20 on page 53). We implement the selection in two steps: first use $a_{i}^{\prime}$ to select two signals from four signals, i.e., performing the following function:

$$
\begin{aligned}
& \text { If } a_{i}^{\prime}=1 \text { then Output }=\left\{b_{1} \text { or } b m_{1}\right\} \\
& \text { Else }\left[a_{i}^{\prime}=0\right] \text { Output }=\left\{0 \text { or } m_{1}\right\}
\end{aligned}
$$

This is supplemented with $q_{i}=\left(a_{i}^{\prime} \cdot b_{0}\right) \oplus r_{0}{ }^{(i-1)}$ to produce the 2-bit selection code needed, as tabulated in Table 3.3:

\begin{tabular}{|c|c|c|}
\hline $\begin{array}{c}\text { Condit } \\
\qquad a^{\prime}{ }_{i}\end{array}$ & $q_{i}=\left(a_{i}^{\prime} \cdot b_{0}\right) \oplus r_{0}^{(i-1)}$ & Operation \\
\hline 0 & 0 & $\mathrm{r}_{1}{ }^{(\mathrm{i})}=\mathrm{r}_{2}{ }^{(\mathrm{i}-1)}+0$ \\
\hline 0 & 1 & $\mathrm{r}_{1}{ }^{(\mathrm{i})}=\mathrm{r}_{2}{ }^{(\mathrm{i}-1)}+\mathrm{m}_{1}$ \\
\hline 1 & 0 & $r_{1}^{(i)}=r_{2}^{(i-1)}+b_{1}$ \\
\hline 1 & 1 & $r_{1}{ }^{(i)}=r_{2}{ }^{(i-1)}+b m_{1}$ \\
\hline
\end{tabular}

TABLE 3.3: Relation between conditions and operation

The implementation of these two selection steps is illustrated in Figure 3.16. 


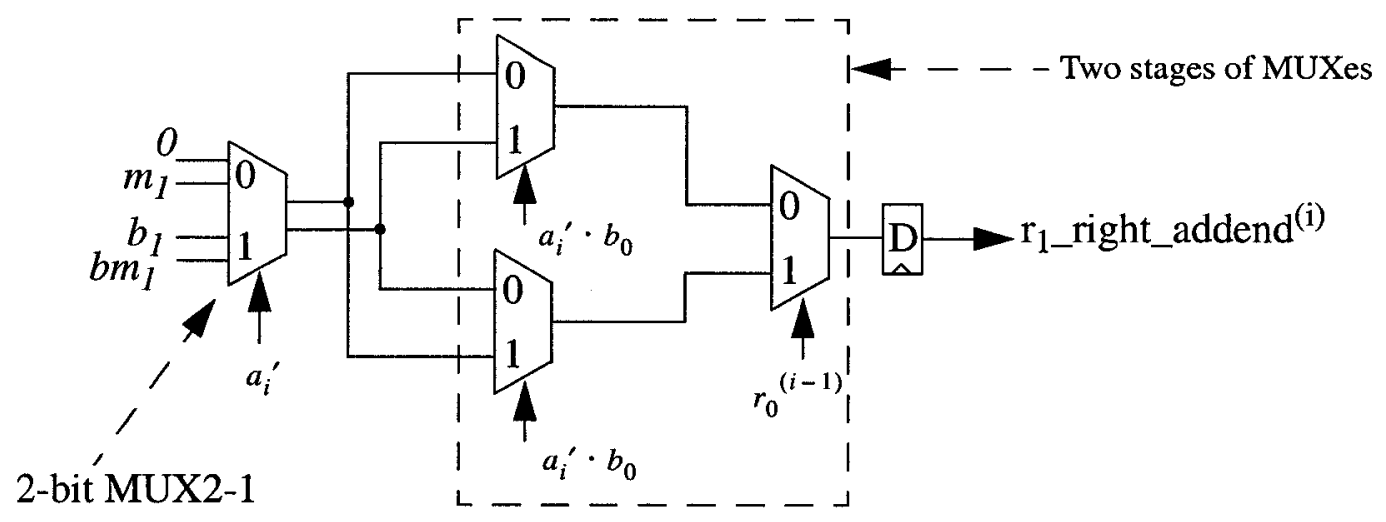

\section{Figure 3.16 Circuit for $r_{1}$ 's right addend}

Five MUX2-1s are employed in Figure 3.16 but do not generate $q_{i}$ as a selection signal. We observed that $r_{0}^{(i-1)}$ is the slowest one among three input signals. If we were to use an XOR gate to generate $q_{i}$ then we would use $q_{i}$ to select $r_{1} \_$right_addend ${ }^{(i)}$, the propagation delay between $r_{0}^{(i-1)}$ and $r_{1}$ right_addend ${ }^{(i)}$ will include the propagation delay of an XOR gate and the propagation delay of a MUX. Instead of generating $q_{i}$, we would use two stages of MUXes to implement the XOR function. Thus, the slowest signal, $r_{0}^{(i-1)}$, is involved at the last selection step. 


\subsubsection{Most Significant Bit Slice}

The most significant bit slice circuit includes one 1-bit full adder and one 2-bit full

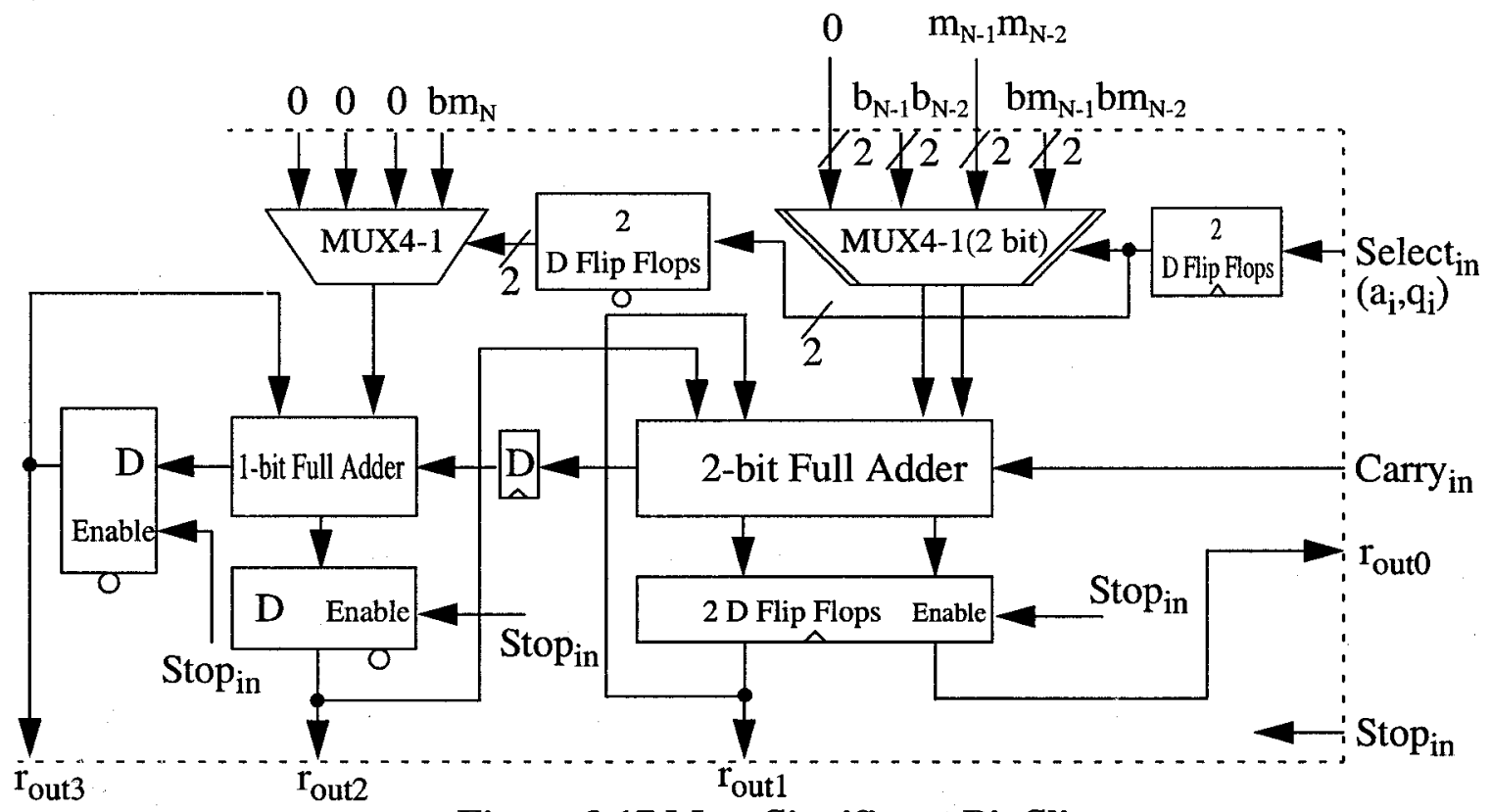

Figure 3.17 Most Significant Bit Slice

adder as shown in Figure 3.17. Because it is the left boundary circuit, it is not necessary to propagate the signals Carry, Stop and Select, to a succeeding bit slice. It must only propagate the data, $r_{\text {out }}$, to its right neighbor.

\subsubsection{Pre-computation Circuit}

The pre-computation circuit generates the selection signals used by MUX4-1. In order to make the critical path delay as small as possible, the selection signal should be supplied to $M U X 4-1$ in advance, causing the corresponding data to arrive at the adder as quickly as possible. The adder can work immediately, without having to wait for the operand from the MUX4-1, and the critical path delay will include only the 2-bit FA's propagation delay.

The key to ensuring that the selection signals in Figure 3.13 on page 47 , i.e. $a_{i}$ and $q_{i}$, are ready in advance, is to pre-generate $q_{i}$ with $a_{i}{ }^{\prime}$. The signal $a_{i}{ }^{\prime}$ will be described in 
section 3.7.9. The pre-computation circuit has four input signals, namely $b_{0}, r_{0}, a_{i}$ and $a_{i}{ }^{\prime}$. Its output signals are $a_{i}$ and $q_{i}$, of which $a_{i}$ is propagated to the output without undergoing any change.

The relationship between input and output is $q_{i}=r_{0}^{(i-1)} \oplus\left(a_{i}^{\prime} \cdot b_{0}\right)$.

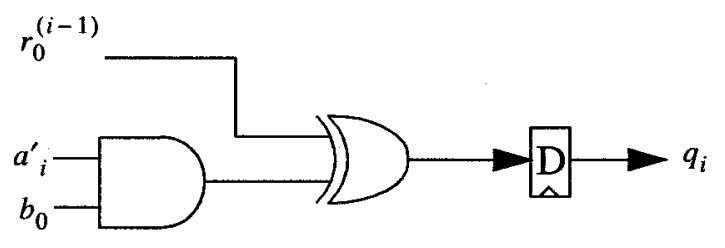

\section{Figure 3.18 Pre-computation circuit}

\subsection{8 'Stop' Signal}

When the target iteration time is reached, a 'Stop' signal (refer to Figure 3.4 on page 34) must be generated to terminate the multiplier iterations. The signal is produced when the algorithm iteration counter $i$ attempts to count past $N-1$. The iteration counter is effectively a clock cycle counter as there is one iteration performed every clock cycle. Figure 3.19 shows how to generate the 'Stop' signal. A 10-bit synchronous binary counter

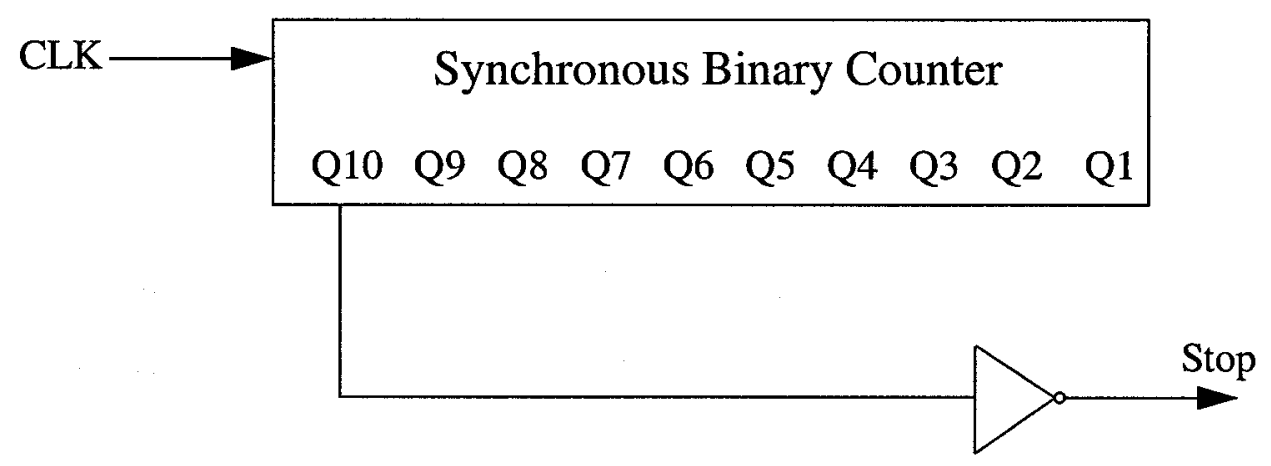

Figure 3.19 "Stop" signal circuit

is used to keep track of the number of clock signals. When this number reaches 1024 , 'Stop' is asserted and the multiplier quits. 
Only a 5-bit and a 7-bit counters are implemented in this work, corresponding to a 32-bit and a 128-bit multipliers respectively. These were used to verify the counter's functioning.

\subsubsection{Shift Register}

The $N$-bit wide shift register of this design has two functions: 'Load' causes an $N$ bit data word to be loaded into the register, and 'Shift' which right-shifts the entire word. It operates in only one direction, right, so that a direction control bit is unnecessary. Figure 3.20 illustrates with a 4-bit shift register having "Shift" and "Load" functions. $a_{i}$ is

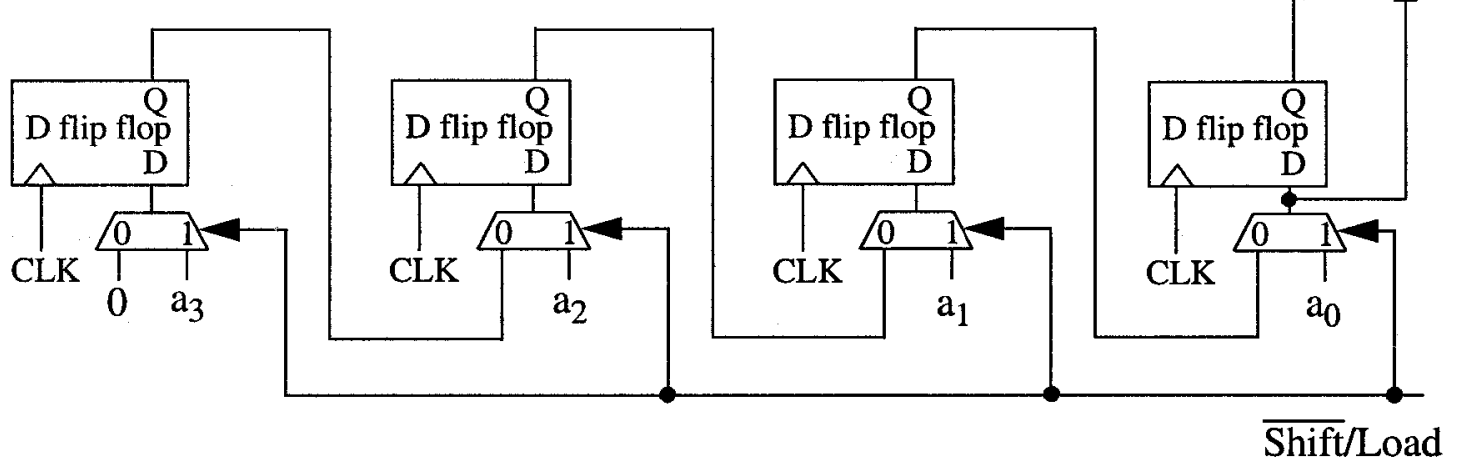

Figure 3.20 4-bit Shift register

just $a_{i}{ }^{\prime}$, delayed by one clock cycle, as shown in Figure 3.20, $a_{i}{ }^{\prime}$ also appears in Figures 3.15 and 3.17 .

\subsection{Full Custom Design}

The custom design was based on a $0.18 \mu \mathrm{m}$ CMOS process. All logic gates, multiplexers (MUX), and full adders are conventional static CMOS logic. Dynamic logic is used for the DFFs. All parts of the multiplier function over a range of 1.0 to 1.8 Volts.

\subsubsection{Multiplexer (MUX)}

The MUXes are conventional static CMOS logic circuits. 
A MUX has $2^{N}$ data inputs, $N$ control inputs and one data output. When $N$ is 1 , it is called $M U X 2-1$, the name indicating two input bits, one output bit, and a selection control bit. The behavioral description of MUX2-1 is

$$
\text { Output }=A \cdot S+B \cdot \bar{S}
$$

where $A, B$ are the two input data and $S$ is the selection signal. The schematic of the CMOS MUX2-1 is shown in Figure 3.21.

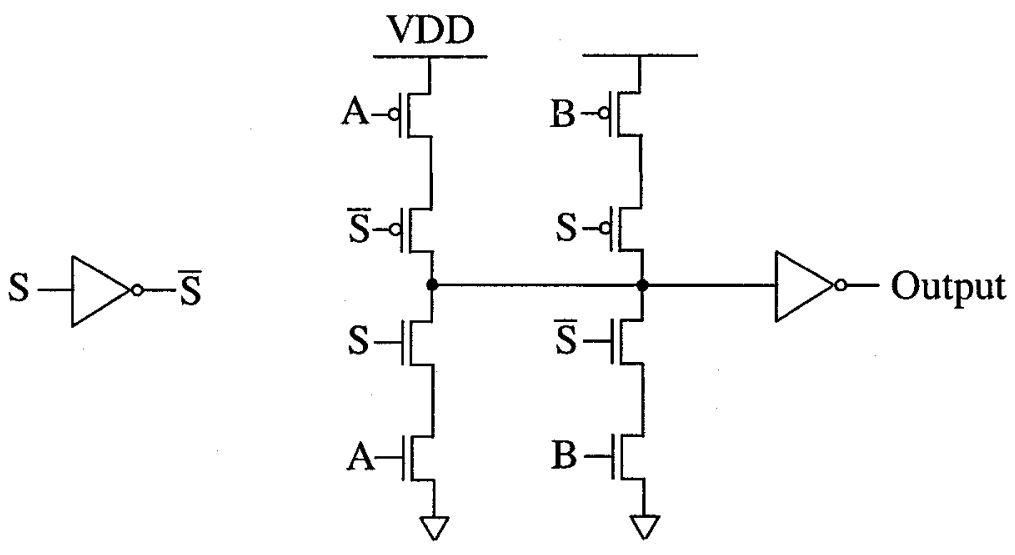

Figure 3.21 CMOS logic MUX2-1 
MUX4-1 is analogous, having four input bits, two selection bits, and one output bit. Its logic equation is output $=A \cdot \overline{S_{0}} \cdot \overline{S_{1}}+B \cdot S_{0} \cdot \overline{S_{1}}+C \cdot \overline{S_{0}} \cdot S_{1}+D \cdot S_{0} \cdot S_{1}$ and the transistor level schematic is shown in Figure 3.22 [30].

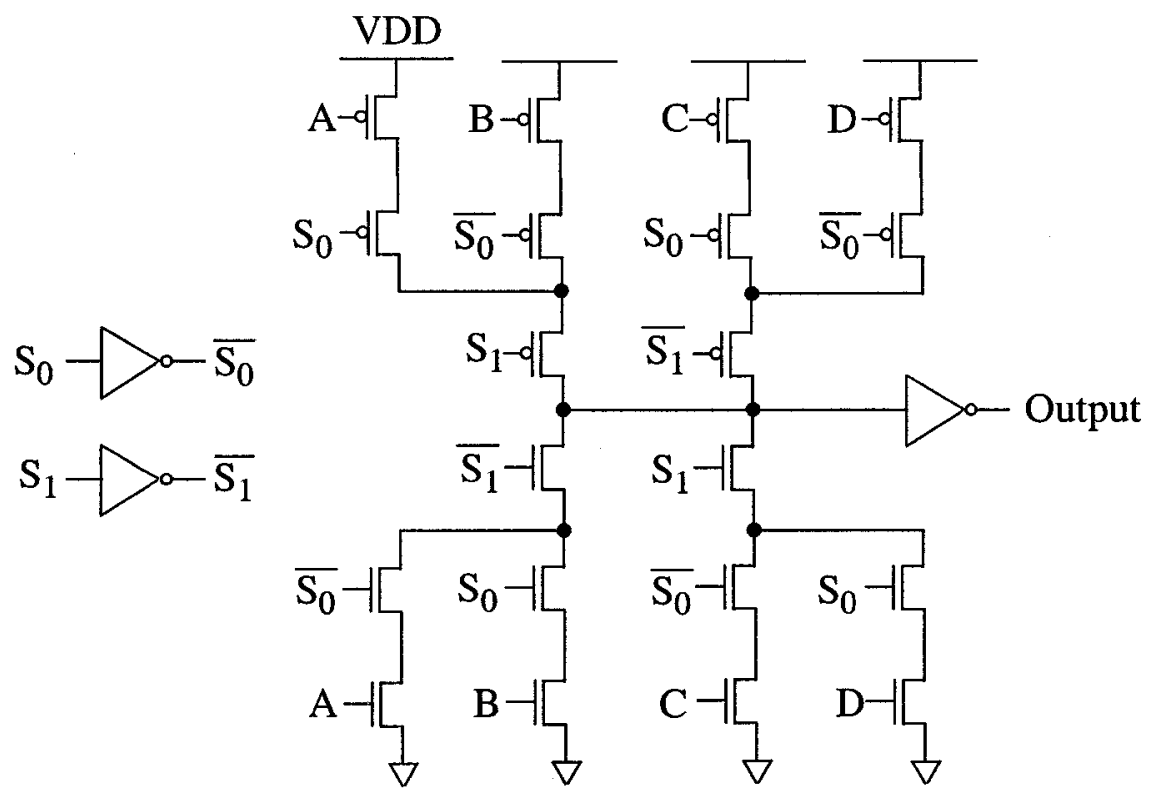

Figure 3.22 CMOS logic MUX4-1

\subsubsection{Full Adder}

Addition is the most common arithmetic operation in digital systems and is often a performance bottleneck [29]. The importance of good performance justifies the effort of full custom design when designing an adder.

In this research work, the 1-bit full adder has a 'mirror' structure which is shown in Figure 3.23 [32]. The completely symmetrical chains of NMOS and PMOS devices display a self-duality property of both the sum and carry functions. As a result, a maximum of two series of transistors can be found in the carry-generation circuitry [29]. The transistors switched by the Carry in signal (C) are near the output of the gate. When 
designing the layout of this full adder, it is most critical that the capacitance at node $\overline{C_{o}}$ be minimized. Shared diffusions are used to help accomplish this end [29].

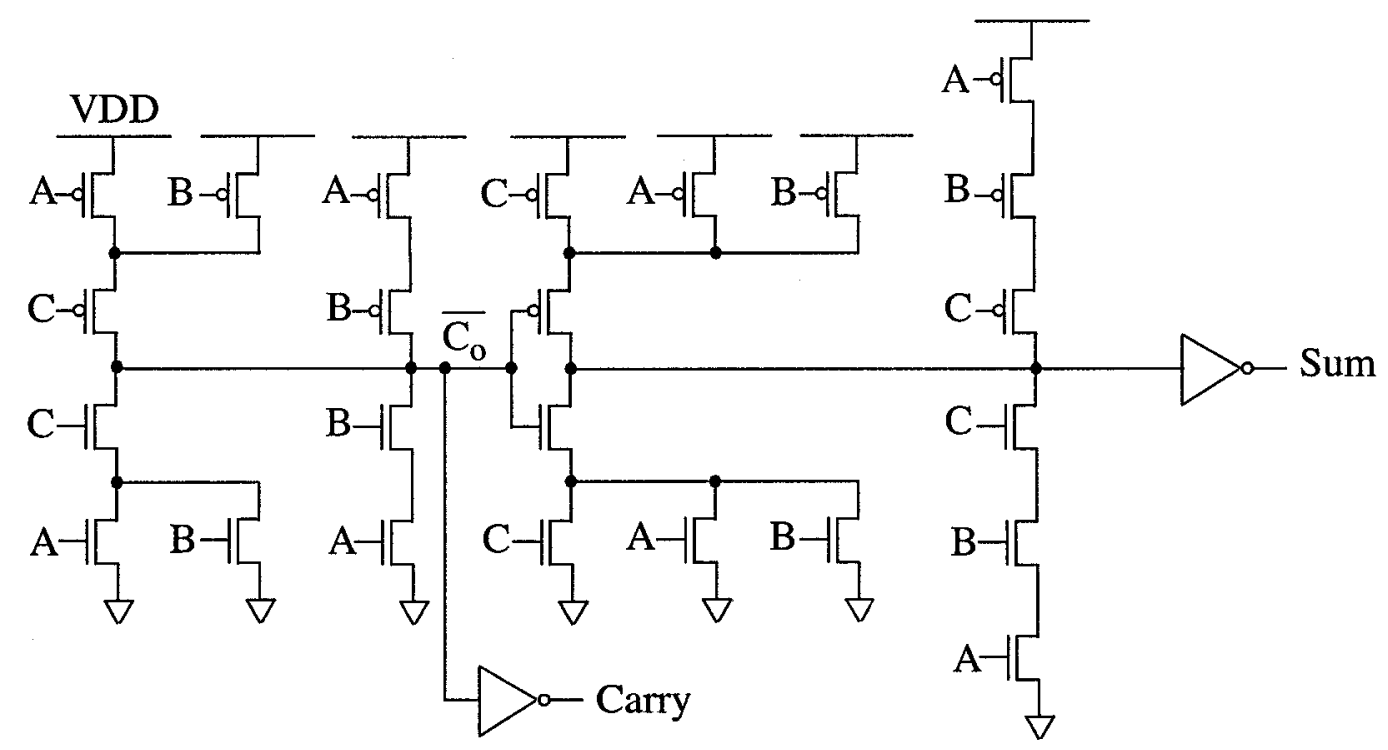

Figure 3.23 Mirror 1-bit full adder

Appendix A describes the self-duality properties of both the sum and carry functions in detail.

Figure 3.24 is the 2 -bit full adder schematic. It has five input signals, $A_{1}, B_{1}, C_{i n}$, $A_{2}$, and $B_{2}$ as well as three output signals, $\operatorname{Sum}_{1}, S u m_{2}$, and $C_{\text {out }}$. This 2-bit full adder consists of three parts. Part (a) generates Sum1. Part (b) generates Carry signal $C_{\text {out }}$. The Carry out signal is generated in two stages: $\overline{C y}$ is the complemented Carry out of the $A_{l}$, $B_{1}, C_{\text {in }}$ addition and Cout combines this with the result of $A_{2}, B_{2}$ addition. Part (c) generates $\mathrm{Sum}_{2}$. Circuits for $\mathrm{Sum}_{1}$ and Cout are optimized to shorten the propagation delay of the entire 2-bit full adder.

Figure 3.25 is a symbolic representation of a 2-bit full adder with Boolean operators. It is observed that the critical path delay of this 2-bit full adder is that of about $2 \mathrm{XOR}$ gates.

The equations for $\bar{C}_{y}, \operatorname{Sum}_{1}, \operatorname{Sum}_{2}$, and $C_{\text {out }}$ are: 


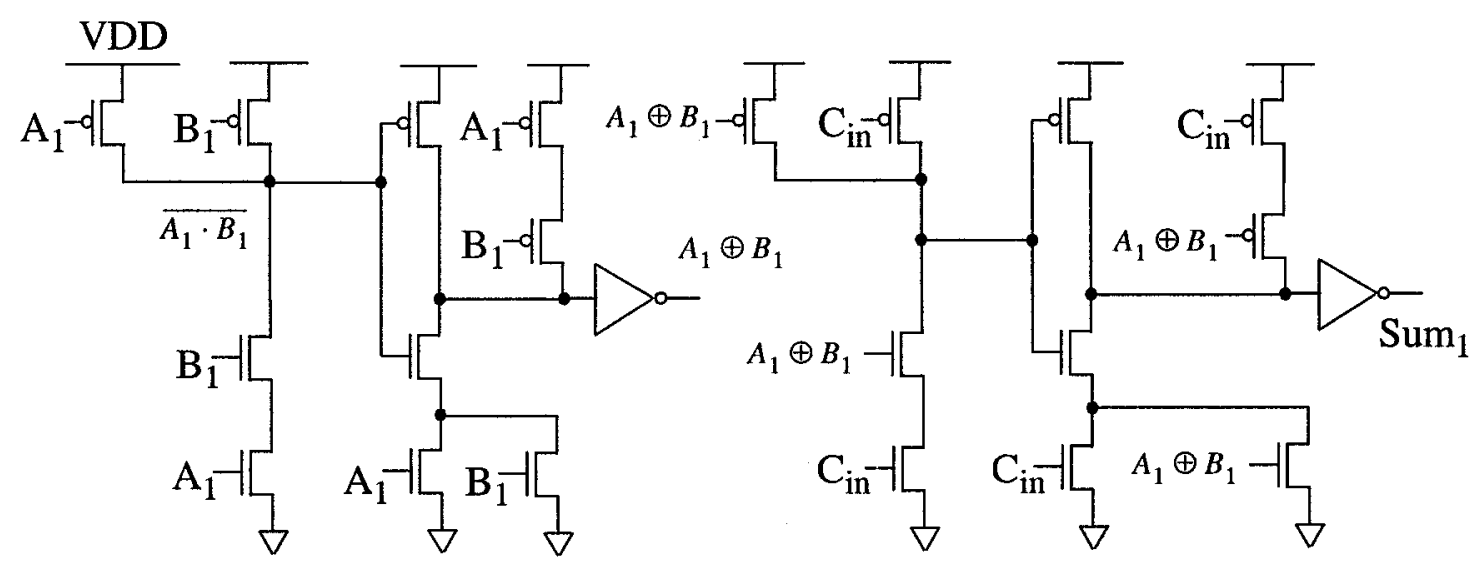

(a) $\mathrm{Sum}_{1}$ circuit
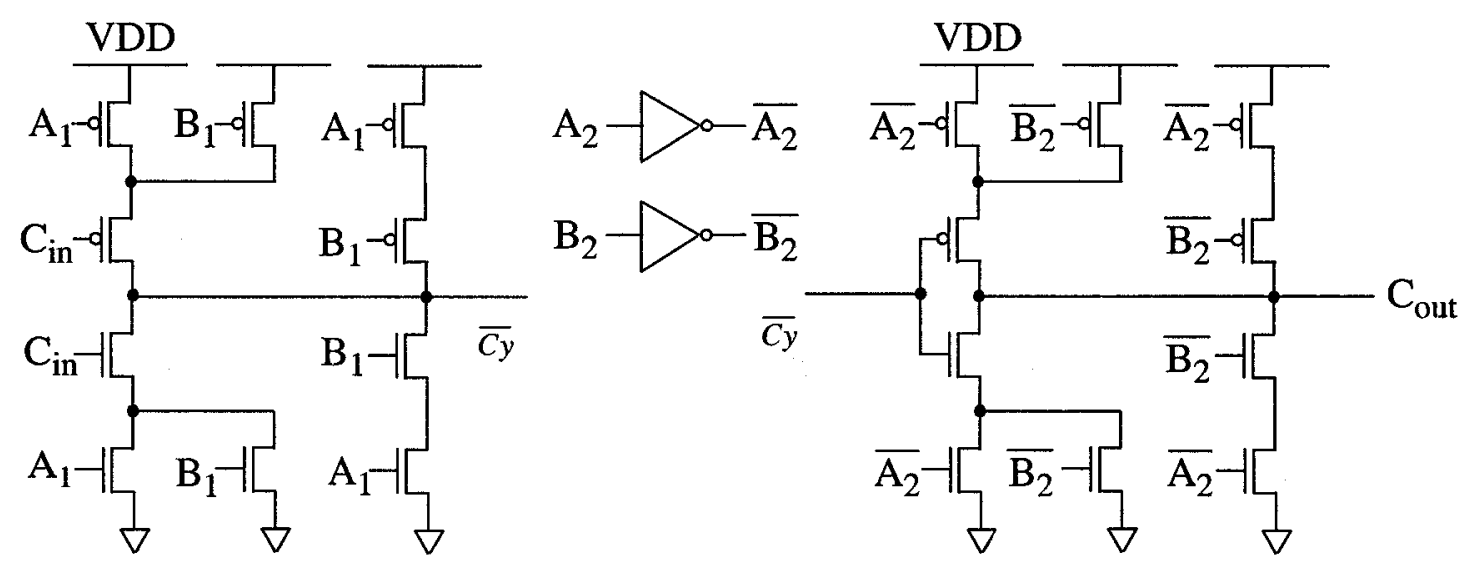

(b) Carry out circuit

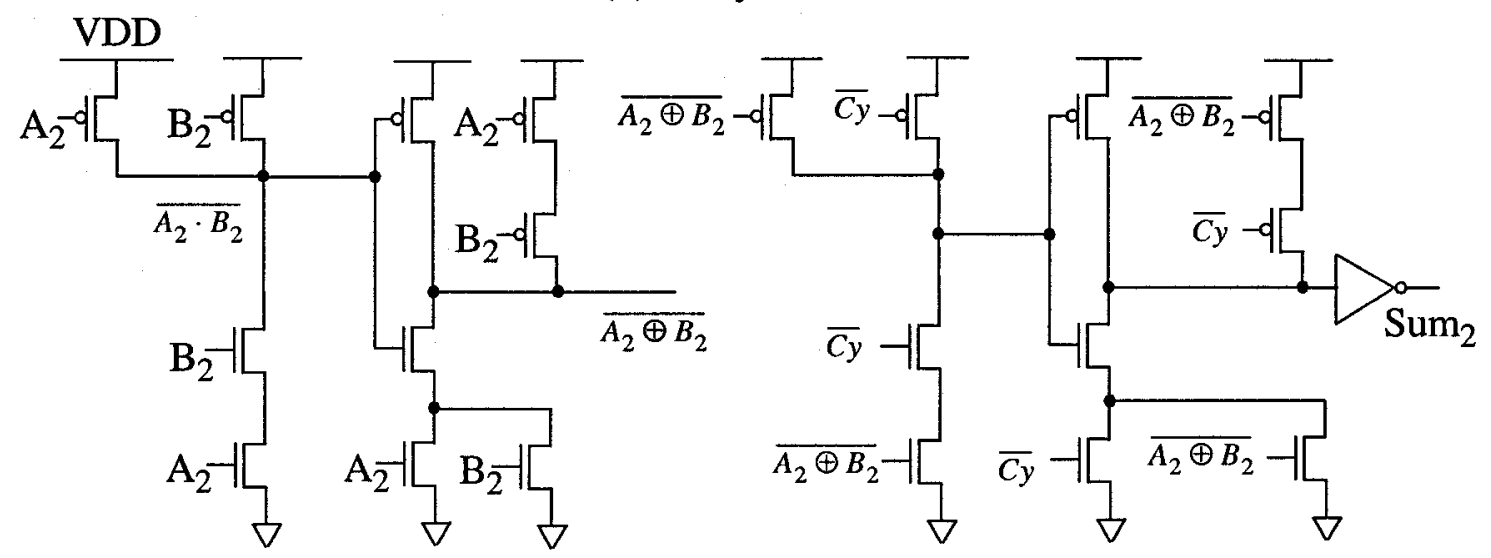

(c) $\mathrm{Sum}_{2}$ circuit

Figure 3.24 2-bit full adder

$\overline{C_{y}}=\overline{A_{1} B_{1}+C_{i n}\left(A_{1}+B_{1}\right)}=\overline{\left(A_{1}+B_{1}\right) \cdot\left(C_{i n}+A_{1} B_{1}\right)}$ (needed internally), 


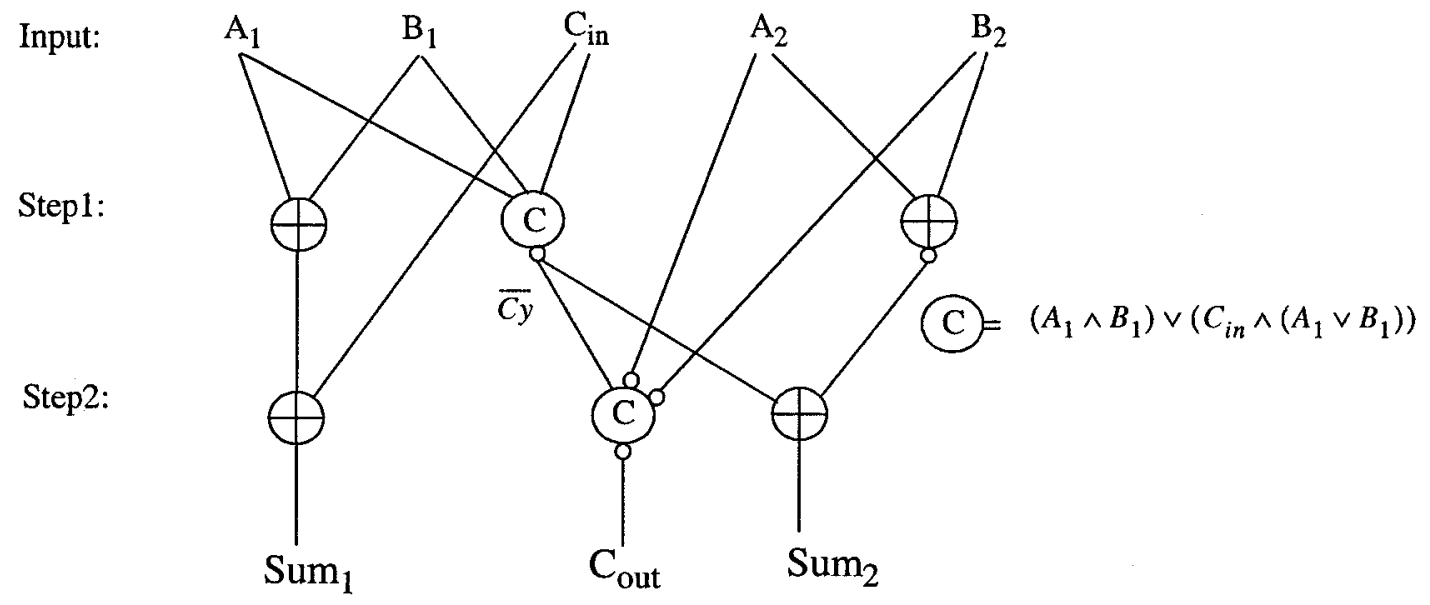

Figure 3.25 Implementation of 2-bit full adder with Boolean operators

$$
\begin{aligned}
& \text { Sum } 1=\left(A_{1} \oplus B_{1}\right) \oplus C_{\text {in }} \text {, where } A_{1} \oplus B_{1}=\overline{\left.\overline{\left(\overline{\left.A_{1}+B_{1}\right) \cdot\left(\overline{A_{1} \cdot B_{1}}\right)}\right.}\right)} \\
& \text { Sum } 2=\overline{A_{2} \oplus B_{2}} \oplus \overline{C_{y}} \text {, where } \overline{A_{2} \oplus B_{2}}=\overline{\left(A_{2}+B_{2}\right) \cdot\left(\overline{A_{2} \cdot B_{2}}\right)} \\
& C_{\text {out }}=\overline{\overline{A_{2}} \overline{B_{2}}+\overline{C_{y}}\left(\overline{A_{2}}+\overline{B_{2}}\right)}
\end{aligned}
$$

The synthesis method of complex CMOS gates [29] is used to transform these equations into the circuits of Figure 3.24.

\subsubsection{Flip Flop and Clock Pulse Generators}

For convenience, we repeat equation 3.2: $T$, the cycle time of a sequential circuit is such that

$$
\mathrm{T}>\mathrm{T}_{\mathrm{p}, \mathrm{comb}}+\mathrm{T}_{\mathrm{cq}}+\mathrm{T}_{\text {setup }}
$$

The term $T_{c q}+T_{\text {setup }}$, also denoted by $T_{d q}$, is determined by the flip flop. In our case, $T_{d q}$ contributes significantly to the total cycle time. In order to reduce $T_{d q}$, we can use a full custom design approach to built faster DFFs.

In recent years, many latches and flip flops have been presented in the literature [33][34]. Among these various types of flip flops, those that have smaller Data-to- $Q$ delays i.e., $T_{d q}$, are more attractive. Traditional master-slave configuration DFFs have two 
stages: one master, one slave. Such a DFF tends to have a positive setup time, an important timing characteristic. Another important DFF parameter is the Clock-to- $Q$ delay, $T_{c q}$, which is the delay time from the clock edge to a stable output [35]. When we calculate the propagation delay caused by traditional DFFs, two parts have to be included: $T_{\text {setup }}$ and $T_{c q}$. Alternatively, pulsed DFFs, short as PDFFs, reduce the two stages to one [36]. As a result, $T_{d q}$ is smaller than that characteristic of a master-slave configuration DFF [36].

The idea of a PDFF is to generate a short pulse around the rising (or falling) edge of the clock signal and then use it to capture the input data. This pulse causes data capture to occur within a very narrow window of time, the pulse width [29]. The very short sampling time helps to prevent race conditions. The pulse-generator/latch combination results in a positive (or negative, depending on the pulse generator's type) edge-triggered DFF.

Figure 3.26 shows schematics of a PDFF and its associated clock pulse generators. In Figure 3.26 (a), node $X$ is called a floating node because it is not always being driven by a transistor, i.e., sometimes all of the transistors connected to this node are turned off. When a node has been floating for a long time, charge decay and noise coupling can cause the node voltage to assume an intermediate value which can lead to a functionality fault [37]. This is not the only way a floating node can cause trouble. In Figure 3.26 (a), node $Q$ is also a floating node, and any CMOS fan-in gates connected to node $Q$ will experience a large short-circuit current when $Q$ is between $V_{t h n}$ and $V_{d d^{-}} V_{t h p}$ because the pull-up and pull-down transistors are simultaneously 'ON'. Floating nodes can be avoided by using 
'keepers' to hold nodes steady. The presence of floating nodes means that the flip flop is a

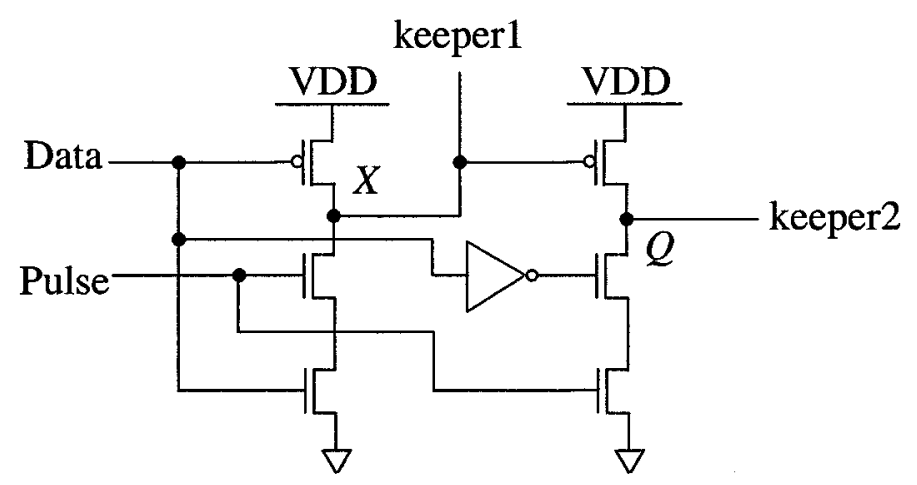

(a) Pulsed D flip flop

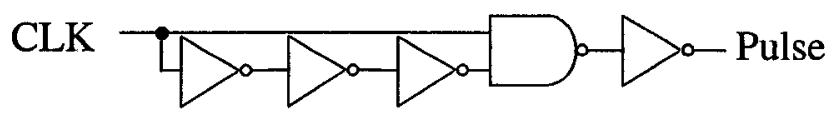

(b) Positive pulse generator

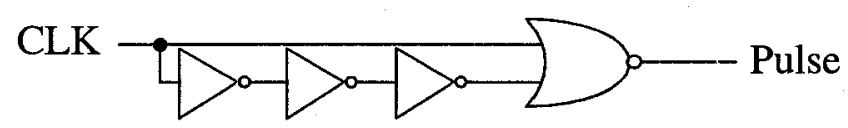

(c) Negative pulse generator

Figure 3.26 Pulsed D Flip Flop and its pulse generators

dynamic type.

Figure 3.27 shows two keeper circuits [37]. Figure 3.27 (a) is used for node $X$ and Figure 3.27 (b) is used for node $Q$ of Figure 3.26 (a). The CMOS pull-up/down keepers ensure that there is no steady short-circuit current path through the keeper transistors regardless of the logic state. The output transistors of the keeper circuits must be weak enough so that the signal drivers can overpower them for proper logic operations. Typically, long channel transistors are used [37].

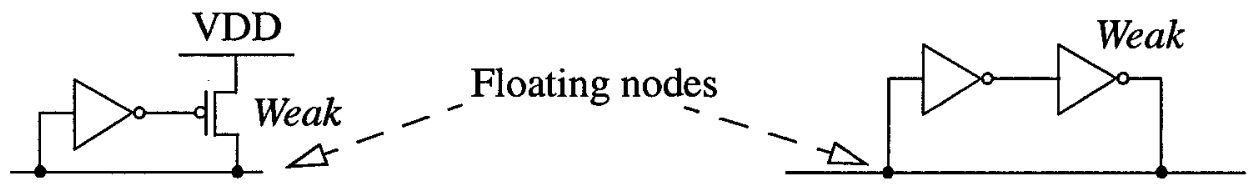

(a) CMOS Pull-up

(b) CMOS Pull-up/down

Figure 3.27 Keeper circuits 


\subsection{Radix-4 Architecture}

\subsubsection{Algorithm}

The architecture discussed until now is based on radix-2. That means in every clock cycle, one bit of the multiplicand, $a_{i}$, is scanned and $a_{i} \times B$ is accumulated. If we want to increase the speed, i.e., shorten the scan time, we can scan 2 bits every clock cycle and accumulate $\left(a_{2 i+1} a_{2 i}\right) \times B$ every clock cycle. Two bits can represent four cases: $0,1,2$, and 3 , and hence the name, 'radix -4 '.

\section{Algorithm 3.3 (Radix-4)}

$\left\{\mathrm{R}^{(-1)}=0 ; 3 \mathrm{~B}=\mathrm{B}+2 \mathrm{~B} ; 3 \mathrm{M}=\mathrm{M}+2 \mathrm{M} ; / / 3 \mathrm{~B}\right.$ and $3 M$ are computed in advance.

\section{For $\mathrm{i}=0$ To $(\mathrm{N} / 2)-1$ \{}

2. Case $\mathrm{a}_{2 \mathrm{i}+1} \mathrm{a}_{2 \mathrm{i}}:\{$

$2.1 \quad 0,0: \quad{R^{\prime}}^{(i)}=R^{(i-1)}+0 ;$

$2.2 \quad 0,1: \quad{R^{\prime}}^{(i)}=R^{(i-1)}+B ;$

$2.3 \quad 1,0: \quad{R^{(i)}}^{(i)} R^{(i-1)}+2 B$;

$\left.2.4 \quad 1,1: \quad{R^{\prime}}^{(i)}=R^{(i-1)}+3 B ;\right\}$

3. Case ${r_{1}}^{(i)}, r_{0}{ }^{(i)}, m_{1}:\{$

$3.10,0, X: R^{(i)}=\left({R^{\prime}}^{(i)}+0\right) / 4$;

$3.20,1,0: R^{(i)}=\left({R^{\prime}}^{(i)}+3 M\right) / 4$;

$3.3 \quad 0,1,1: R^{(i)}=\left({R^{(i)}}^{(i)}+M\right) / 4 ;$

$3.4 \quad 1,0, X: R^{(i)}=\left({R^{\prime}}^{(i)}+2 M\right) / 4 ;$

$3.5 \quad 1,1,0: R^{(i)}=\left({R^{(i)}}^{(i)} M\right) / 4 ;$

$\left.3.6 \quad 1,1,1: R^{(i)}=\left({R^{\prime}}^{(i)}+3 M\right) / 4 ;\right\}$

\} 


\section{Return R;}

\}

Note: $X$ means don't care.

\subsubsection{High Level Architecture}

A somewhat abstract radix-4 hardware architecture is shown in Figure 3.28.

Thick lines represent multi-bit data lines. Thin lines represent 1 -bit data lines

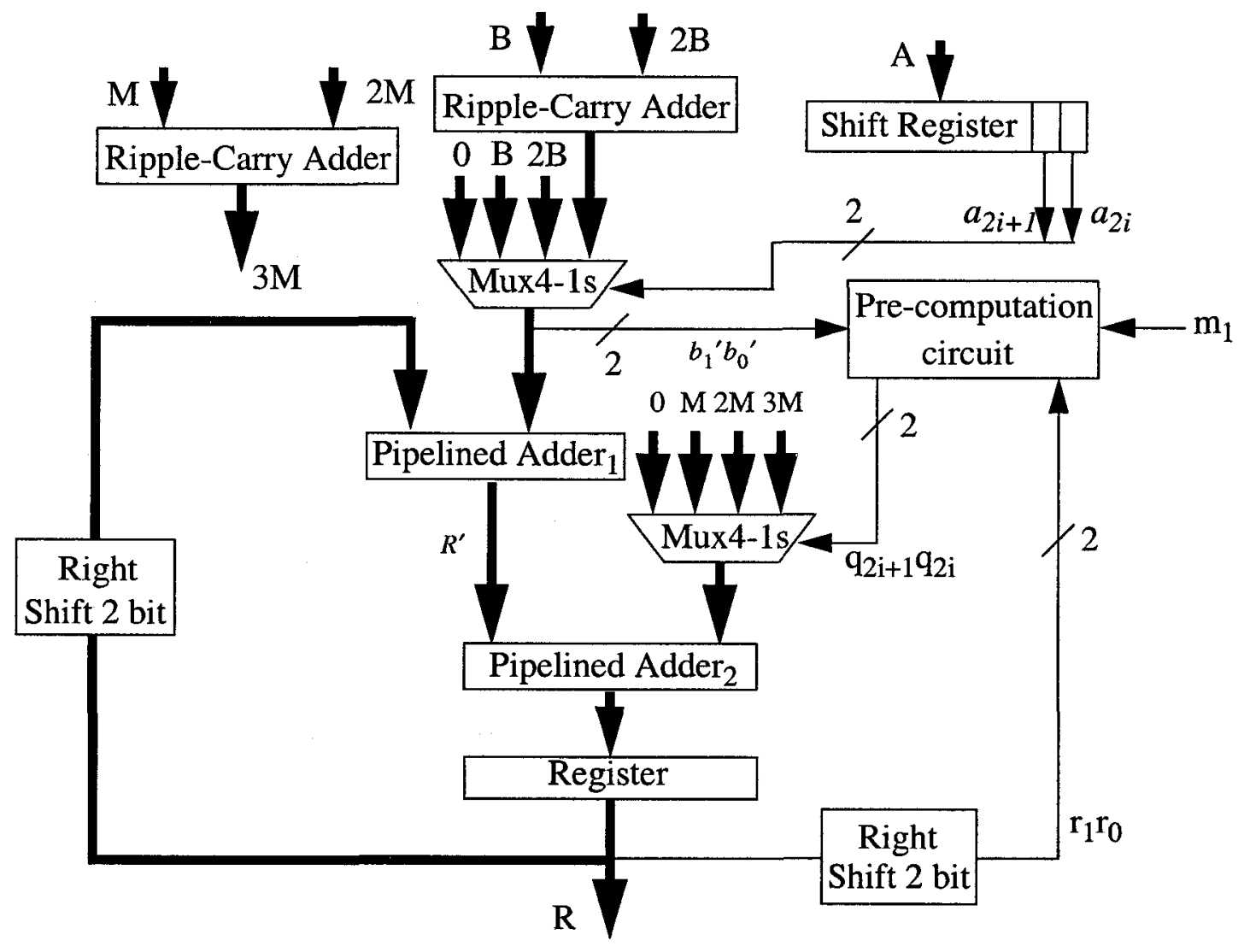

Figure 3.28 Radix-4 architecture

unless indicated otherwise by a number. It uses two ripple-carry adders and two pipelined adders. Two ripple-carry adders are used to generate $3 M$ and $3 B$ respectively. Here we suppose the input data $(B$ and $M$ ) are stable during the whole of the computation. Like the radix-2 version, it uses a pre-computation circuit to generate the selection signals, i.e. $q_{2 i+1} q_{2 i}$. In Figure 3.28, $b_{0}{ }^{\prime}$ and $b_{1}{ }^{\prime}$ are two LSBs of the MUX4 output. In every iteration, 
it computes two additions: one to compute $R+\left(a_{2 i+1} a_{2 i}\right) \times B$ and the other to compute $R^{\prime}+\left(q_{2 i+1} q_{2 i}\right) \times M$. The products $\left(a_{2 i+1} a_{2 i}\right) \times B$ and $\left(q_{2 i+1} q_{2 i}\right) \times M$ are selected by MUX4-1 outputs.

The first addition, produces the intermediate result $R^{\prime}$. Based on the two LSBs of ${R^{\prime}}^{(i)}$, i.e., $r_{1}{ }^{\prime} r_{0}{ }^{(i)}, q_{2 i+1} q_{2 i}$ can be determined. Table 3.4 summarizes the calculation of $q_{2 i+1} q_{2 i}$, and the corresponding operations.

TABLE 3.4: Determination of $\mathbf{q}_{2 \mathrm{i}+1} \mathbf{q}_{2 \mathrm{i}}$ and the corresponding operations

\begin{tabular}{|c|c|c|c|}
\hline $\mathbf{r}_{1}{ }_{1} \mathbf{r}^{\prime}(\mathbf{i})$ & $\mathbf{m}_{\mathbf{1}} \mathbf{m}_{\mathbf{0}}$ & $\mathbf{q}_{2 \mathrm{i}+\mathbf{1}} \mathbf{q}_{2 \mathrm{i}}$ & $\begin{array}{l}\text { Operations } \\
\mathbf{R}^{(\mathbf{i})}=\left(\mathbf{R}^{,(\mathbf{i})}+\left(\mathbf{q}_{2 \mathrm{i}+\mathbf{1}} \mathbf{q}_{2 \mathbf{i}}\right) \times \mathbf{M}\right) / 4\end{array}$ \\
\hline 00 & Don't care & $\overline{00}$ & $R^{(i)}=\left(R^{(i)}+0\right) / 4$ \\
\hline 01 & 01 & 11 & $R^{(i)}=\left({R^{\prime}}^{(i)}+3 \times M\right) / 4$ \\
\hline 01 & 11 & 01 & $R^{(i)}=\left(R^{\prime(i)}+M\right) / 4$ \\
\hline 10 & Don't care & 10 & $R^{(i)}=\left(R^{\prime(i)}+2 \times M\right) / 4$ \\
\hline 11 & 01 & 01 & $R^{(i)}=\left(R^{\prime(i)}+M\right) / 4$ \\
\hline 11 & 11 & 11 & $R^{(i)}=\left({R^{\prime}}^{(i)}+3 \times M\right) / 4$ \\
\hline
\end{tabular}

A Karnaugh map can be used to express the relationship between $r_{l}^{(i-1)}, r_{0}^{(i-1)}$, $b_{1}{ }^{\prime}, b_{0}{ }^{\prime}, m_{1}$, and $q_{2 i+1} q_{2 i}$. The relations are written out in Table $3.5\left(q_{2 i}\right)$ and in Table 3.6 $\left(q_{2 i+1}\right)$.

TABLE 3.5: Karnaugh map of $q_{2 i}$

\begin{tabular}{|c|c|c|c|c|c|c|c|c|}
\hline $\mathrm{r}_{1} \mathrm{r}_{0}^{(\mathrm{i}-1)} b_{1}^{\prime} b_{0}{ }^{\prime} m_{1}$ & 000 & 001 & 011 & 111 & 101 & 100 & 110 & 010 \\
\hline 00 & 0 & 0 & 1 & 1 & 0 & 0 & 1 & 1 \\
\hline 01 & 1 & 1 & $\overrightarrow{0}$ & 0 & 1 & 1 & 0 & 0 \\
\hline 11 & 1 & 1 & $\mathbf{0}$ & 0 & 1 & 1 & 0 & 0 \\
\hline 10 & 0 & 0 & $\sqrt{1}$ & 1 & 0 & 0 & 1 & 1 \\
\hline
\end{tabular}

From Table 3.5, we can get $q_{2 i}=r_{0}{ }^{(i-1)} \overline{b_{1}^{\prime}} \overline{b_{0}^{\prime}}+r_{0}^{(i-1)} b_{1}^{\prime} \overline{b_{0}^{\prime}}+\overline{r_{0}{ }^{(i-1)}} b_{0}^{\prime} m_{1}+\overline{r_{0}{ }^{(i-1)}} b_{0}^{\prime} \overline{m_{1}}$ which can be simplified to: 


$$
q_{2 i}=r_{0}^{(i-1)} \oplus b_{0}^{\prime}
$$

For the more significant bit, $q_{2 i+1}$, the expression is not so easy as for $q_{2 i}$ because Karnaugh maps are troublesome for functions having five and more variables. However, we can consider the contents of the cells as variables and not only the constants 0 and 1 . In Table 3.6, consider the contents in the first, second, third and forth rows as variable $\boldsymbol{A}, \boldsymbol{B}$, $\boldsymbol{C}$, and $\boldsymbol{D}$ respectively. Each variable can be described by a smaller Karnaugh map.

TABLE 3.6: Karnaugh map of $\mathbf{q}_{2 \mathbf{i}+1}$

\begin{tabular}{|c|c|c|c|c|c|c|c|c|}
\hline $\mathrm{r}_{1} \mathrm{r}_{0}{ }^{\prime} b_{0}{ }^{(i-1)}$ & 000 & 010 & 110 & 100 & 101 & 111 & 011 & 001 \\
\hline 00 & 0 & 1 & 0. & 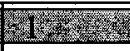 & (2) & In & 65 & 0 \\
\hline \begin{tabular}{|l|}
01 \\
\end{tabular} & 1 & 1 & 0 & 0 & 1 & 0 & 1 & 0 \\
\hline 11 & 0 & 16 & 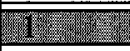 & lis & a & Sis & 100 & 19 \\
\hline 10 & 1 & 0 & 1 & 0 & 0 & $\overline{0}$ & 1 & $\overline{1}$ \\
\hline
\end{tabular}

Table 3.7 through Table 3.10 are the expressions of variable $\boldsymbol{A}, \boldsymbol{B}, \boldsymbol{C}$ and $\boldsymbol{D}$ respectively.

TABLE 3.7: Karnaugh map for variable $A$

\begin{tabular}{|l|l|l|l|l|}
\hline$b_{1}^{\prime}$ & $b_{0}{ }^{\prime} m_{1}$ & \multicolumn{4}{l}{} \\
\hline 0 & 00 & 01 & 11 & 10 \\
\hline 1 & 0 & 0 & 0 & 1 \\
\hline
\end{tabular}

TABLE 3.8: Karnaugh map for variable $B$

\begin{tabular}{|l|l|l|l|l|}
\hline$b_{1}{ }^{b_{0}{ }^{\prime} m_{1}}$ & \multicolumn{4}{|c|}{} \\
\hline 0 & 00 & 01 & 11 & 10 \\
\hline 1 & 1 & 0 & 1 & 1 \\
\hline
\end{tabular}

TABLE 3.9: Karnaugh map for variable $C$

\begin{tabular}{|l|l|l|l|l|}
\hline$b_{1}{ }^{b_{0}{ }^{\prime} m_{1}}$ & \multicolumn{4}{|c|}{} \\
\hline 0 & 00 & 01 & 11 & 10 \\
\hline 1 & 0 & 1 & 0 & 0 \\
\hline
\end{tabular}


TABLE 3.10: Karnaugh map for variable $D$

\begin{tabular}{|l|l|l|l|l|}
\hline$b_{1}^{\prime}$ & $b_{0}{ }^{\prime} m_{1}$ & \multicolumn{4}{l|}{} \\
\hline 0 & 00 & 01 & 11 & 10 \\
\hline 1 & 0 & 0 & 0 & 1 \\
\hline
\end{tabular}

Circuit implementations of Table 3.7 - Table 3.10 are simple. Using with a MUX41, the circuit for the generation of $q_{2 i+1} q_{2 i}$ can be implemented as in Figure 3.29.

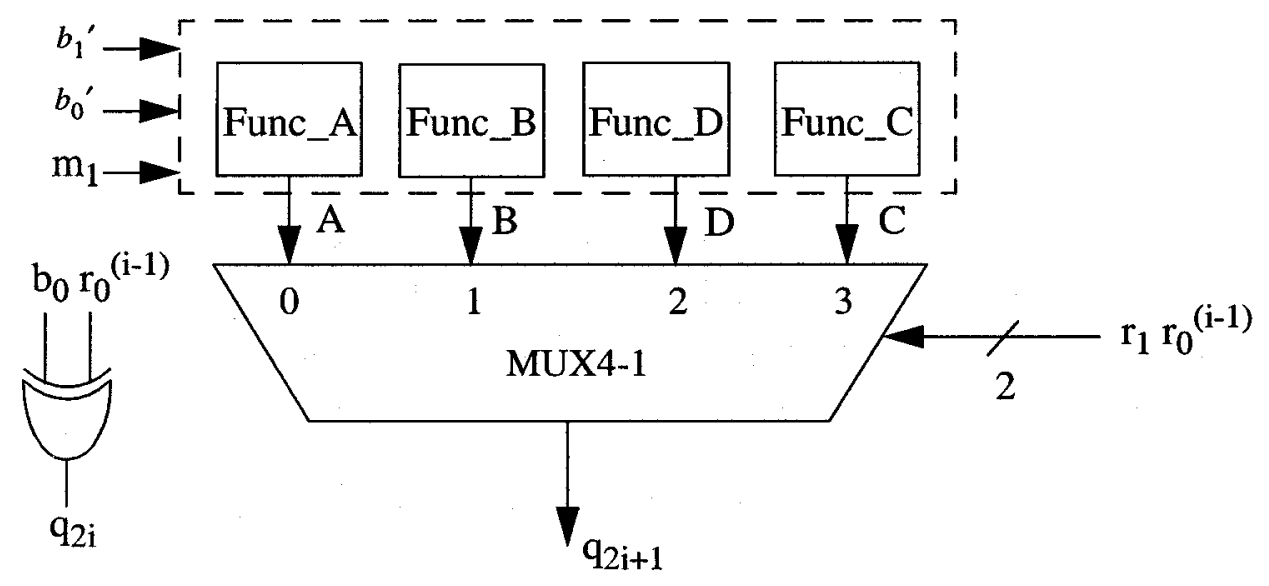

Figure 3.29 Generation of $q_{2 i+1} q_{2 i}$ bit pair

\subsubsection{Clock Signals}

A two-phase clock is used in the radix-4 architecture. One phase triggers the DFFs in the Pipelined Adder ${ }_{1}$ of Figure 3.28 and the other phase is for other sequential components.

\subsubsection{Radix-4 Standard Bit Slice}

Figure 3.30 shows the standard radix -4 multiplier bit slice. The operation of this cell has two main steps: one to calculate $R+B$, the other to calculate $M+(R+B)$. The prod- 
ucts of $B$ and $M$ are selected by two $M U X 4-1 s$ respectively. Seven 2-bit possible inputs,

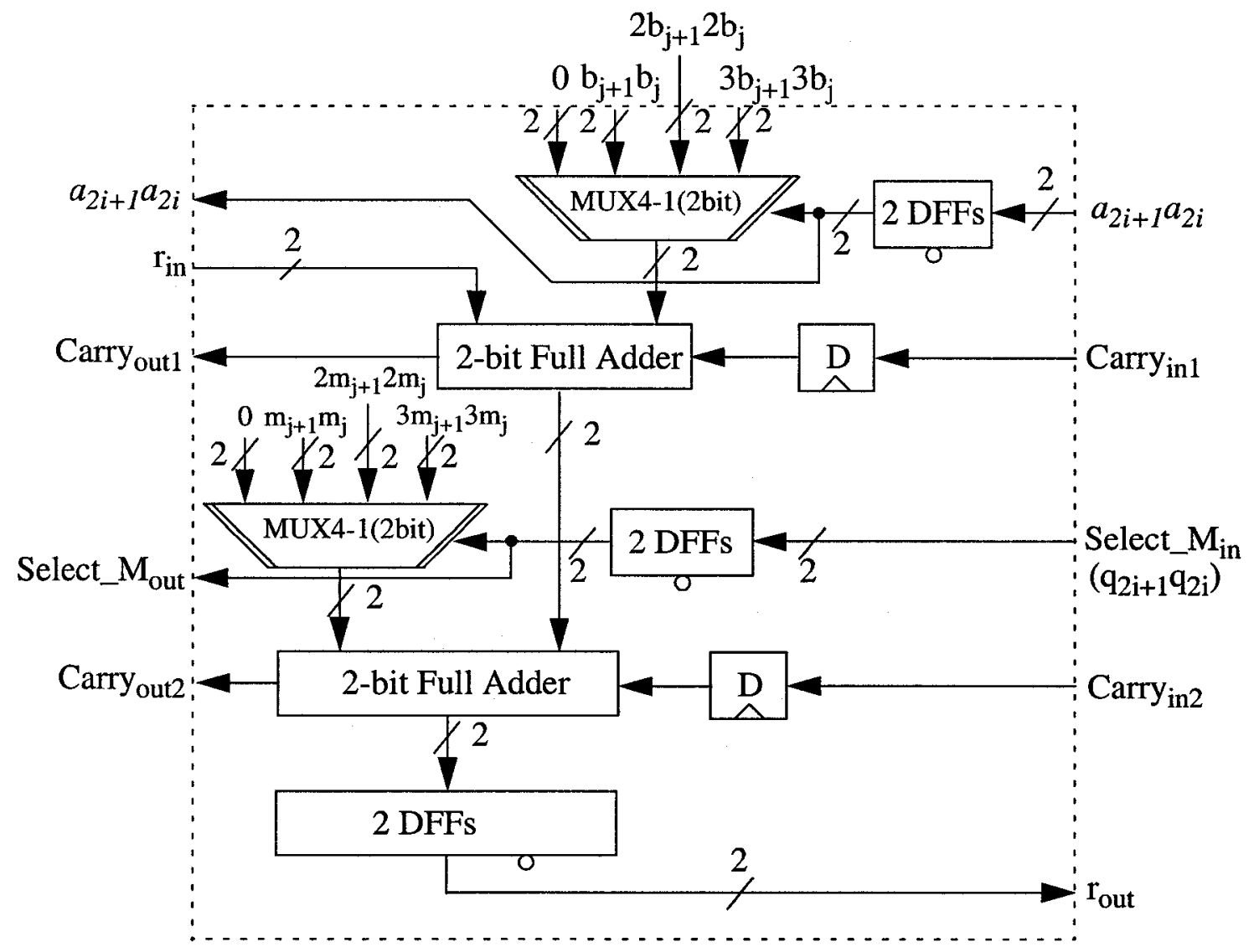

Figure 3.30 Standard bit slice of radix-4 architecture

namely $0, b_{j+1} b_{j}, 2 b_{j+1} 2 b_{j}$, and $3 b_{j+1} 3 b_{j}$ as well as $m_{j+1} m_{j}, 2 m_{j+1} 2 m_{j}$, and $3 m_{j+1} 3 m_{j}$ appear at the top of the diagram. $2 b_{j+1} 2 b_{j}$ and $2 m_{j+1} 2 m_{j}$ are the $j+1$ th and $j$ th bits of $2 B$ and $2 M$ respectively. Similarly, $3 b_{j+1} 3 b_{j}$ and $3 m_{j+1} 3 m_{j}$ are the $j+1$ th and $j$ th bits of $3 B$ and $3 M$ respectively. 'Select_ $M$ ' signals, i.e. $q_{2 i+1} q_{2 i}$, as well as $a_{2 i+1} a_{2 i}$ appear at the righthand side and are propagated to the cell's left-hand neighbors. $r_{i n}$ is the result bit pair of the left neighbor cell, and $r_{\text {out }}$ is output bit pair fed to the right neighbor cell.

Similar to the radix-2 architecture, there are two types of standard bit slice circuits which are distinguished by different edge triggered D flip flops. These two type circuit are arranged in an alternating fashion, just like the radix-2 architecture. 


\subsubsection{Shift Register}

A 6-bit shift register is shown in Figure 3.31. This shift register right-shifts 2 bits in one clock cycle. $a_{2 i+1}\left(Q_{1}\right)$ and $a_{2 i}\left(Q_{0}\right)$ are two output signals of this shift register. The

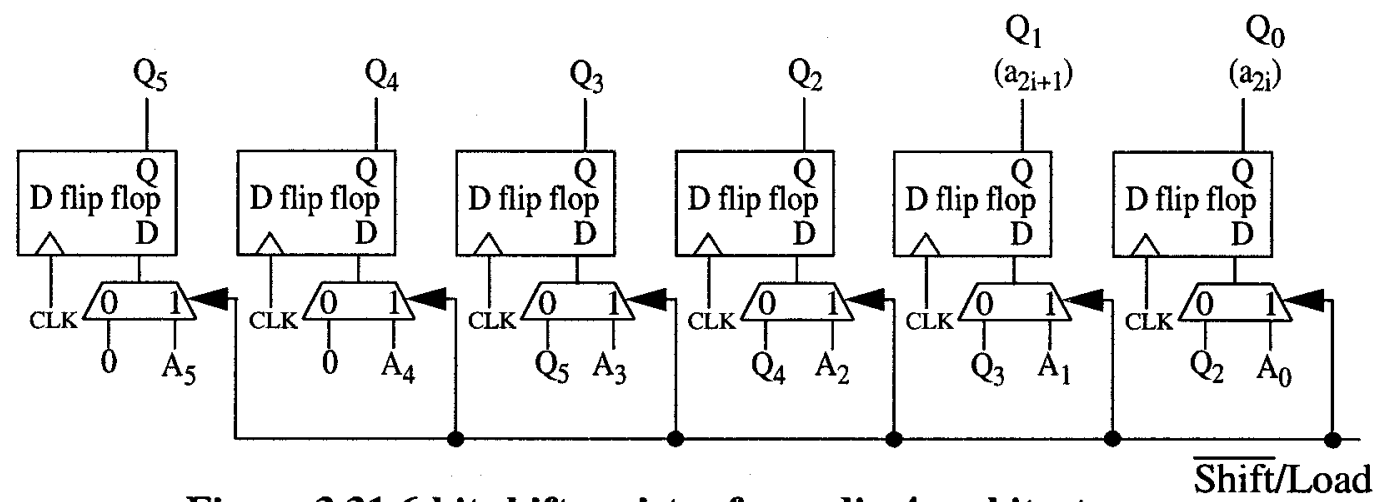

Figure 3.31 6-bit shift register for radix-4 architecture

circuit of Figure 3.31 moves two bits per clock, as opposed to the one bit per clock of Figure 3.20.

\subsection{Summary}

The hardware implementations of radix -2 and radix -4 versions of the Montgomery algorithm have been presented in this chapter. The architectures employ two types of edge triggered D flip flops and use a new clocking scheme to achieve results in fewer clock cycles than do existing designs.

The simulation results will be presented in the next chapter. 
This chapter compares some simulation results with the behavior of previously reported modular multipliers.

We begin with the results of post-layout simulation of $0.18 \mu \mathrm{m}$ CMOS implementation of two important multiplier components - the custom designed pulsed D flip flop (PDFF) and the 2-bit full adder - and then compare these with those of their counterparts in a standard cell library. This is followed by simulation of the 32-bit Montgomery modular multiplier to be used in the present design. These results are compared with the known characteristics of other reported Montgomery modular multipliers.

\subsection{Montgomery Modular Multiplier Primitives}

\subsubsection{Pulsed D Flip Flop}

We use "Data" and " $Q$ " to denote the signals present at the input and output of a PDFF. The critical parameter of a PDFF is the Data-to- $Q$ delay $\left(T_{d q}\right)$ because it is equal to the fraction of the clock cycle time required by the PDFF [33]. If instead the Clock-to- $Q$ delay $\left(T_{c q}\right)$ were to be used, the PDFF setup time (the Data-to-Clock delay, $T_{\text {setup }}$ ), would not be accounted for [33]. 
The PDFF's $T_{d q}$ is found by progressively skewing the input data with respect to the clock edge until the circuit fails. Figure 4.1 shows the timing relationship between $T_{d q}$ and $T_{\text {setup }}$

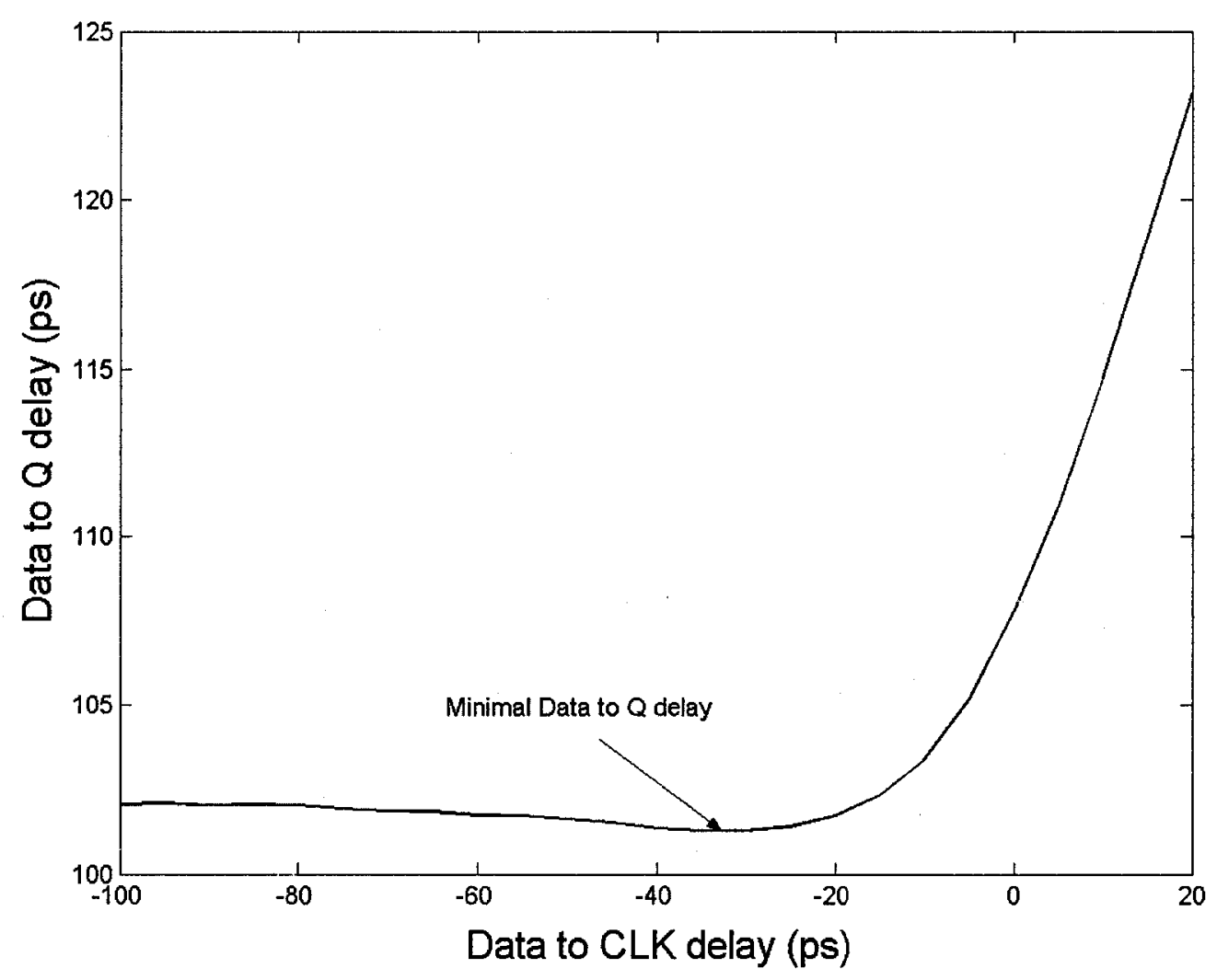

Figure 4.1 Data-to- $Q$ delay of pulsed D flip flop

In Figure 4.1, we can see that $T_{d q}$ is about $102 \mathrm{ps}$. The simulation was done with the PDFF output driving a $6 \mathrm{fF}$ capacitor.

In order to compare a standard cell library D flip flop to the proposed one, a test bench was built to obtain the critical specifications of these $D$ flip flops.

Table 4.1 compares observed parameters of the proposed PDFF to those of a library unit named DFEPQ1, when the clock period is $2 \mathrm{~ns}$. They are all positive edge trig- 
gered D flip flops. We can see that our PDFF has a $T_{d q}$ which is much faster than that of its

TABLE 4.1: Power and speed comparison (same load conditions)

\begin{tabular}{|c|c|c|c|}
\hline D flip flop & Power & Data to $Q$ delay $\left(T_{d q}\right)$ & $T_{d q}$ in Databook \\
\hline Pulsed D flip flop & $0.486 \mathrm{~mW}$ (3 D FFs) & 122.92 ps (Fall) 98.81 ps (Rise) & N/A \\
\hline DFEPQ1 & $0.403 \mathrm{~mW}$ (3 D FFs) & $422.78 \mathrm{ps}$ (Fall) $391.04 \mathrm{ps}$ (Rise) & $479 \mathrm{ps}$ (Fall) $303 \mathrm{ps}$ (Rise) \\
\hline
\end{tabular}

library counterpart.

The power consumption of our PDFF exceeds that of the DFEPQ1 by $20.6 \%$.

Figure 4.2 shows the results of transient simulations. In the top panel of Figure 4.2, the shortest setup times consistent with correct operation of the two DFFs were used. In the bottom panel of the figure, the left waveform is the input data (Data), and the other two are the outputs of two DFFs $(Q)$. It can be seen that Data comes after the clock edge 
of the PDFF. As indicated at the bottom of the figure, the proposed PDFF is 299.907 ps Transient Response

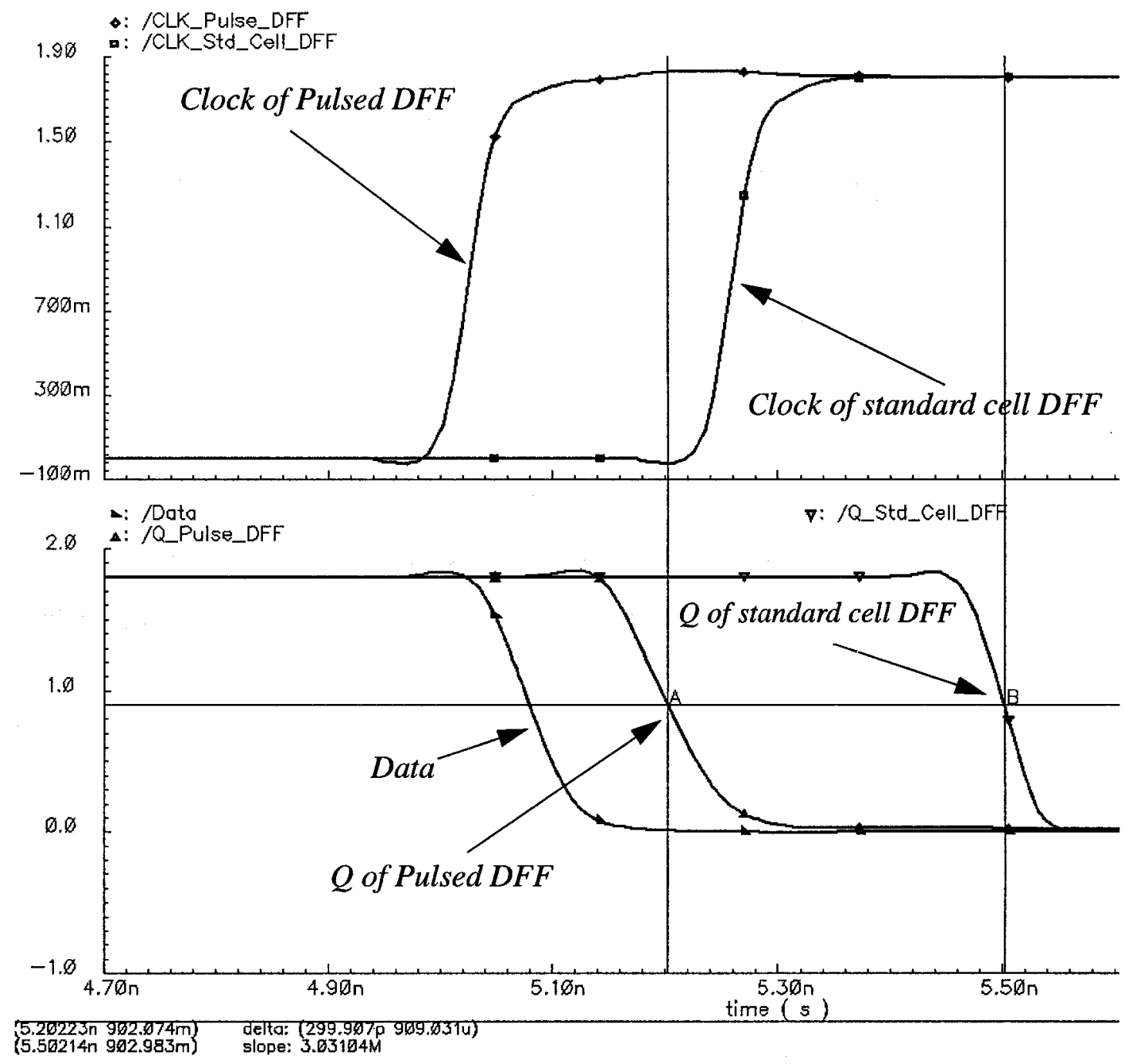

Figure 4.2 Comparison of Data-to- $Q$ delay

faster than the library device.

\subsubsection{2-bit Full Adder}

Only the least significant bit is processed by a 1-bit full adder. This part of the circuit is not in the critical path. But the remaining 2-bit full adders are, and so these must be carefully optimized. The paths involving Carry_out $\left(\mathrm{C}_{\text {out }}\right)$ and $S_{u m}$ were optimized. 
Figure 4.3 shows the 2-bit full adder's Carry circuit before optimization.

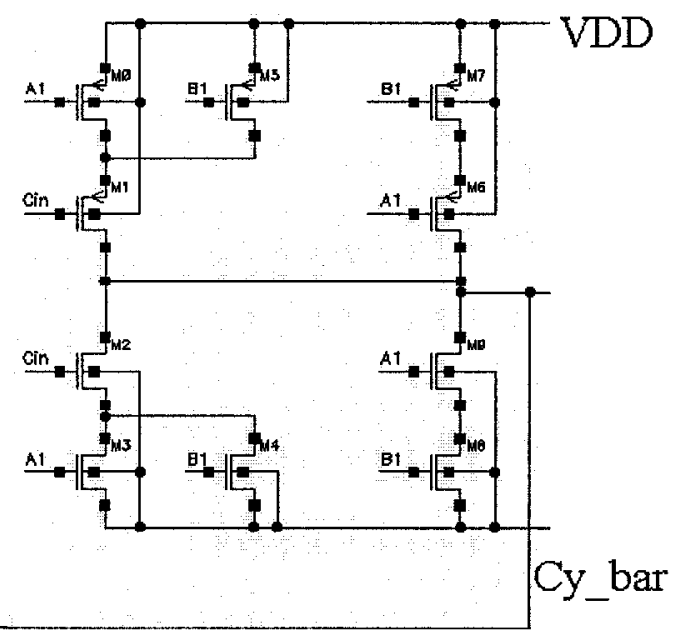

(a)

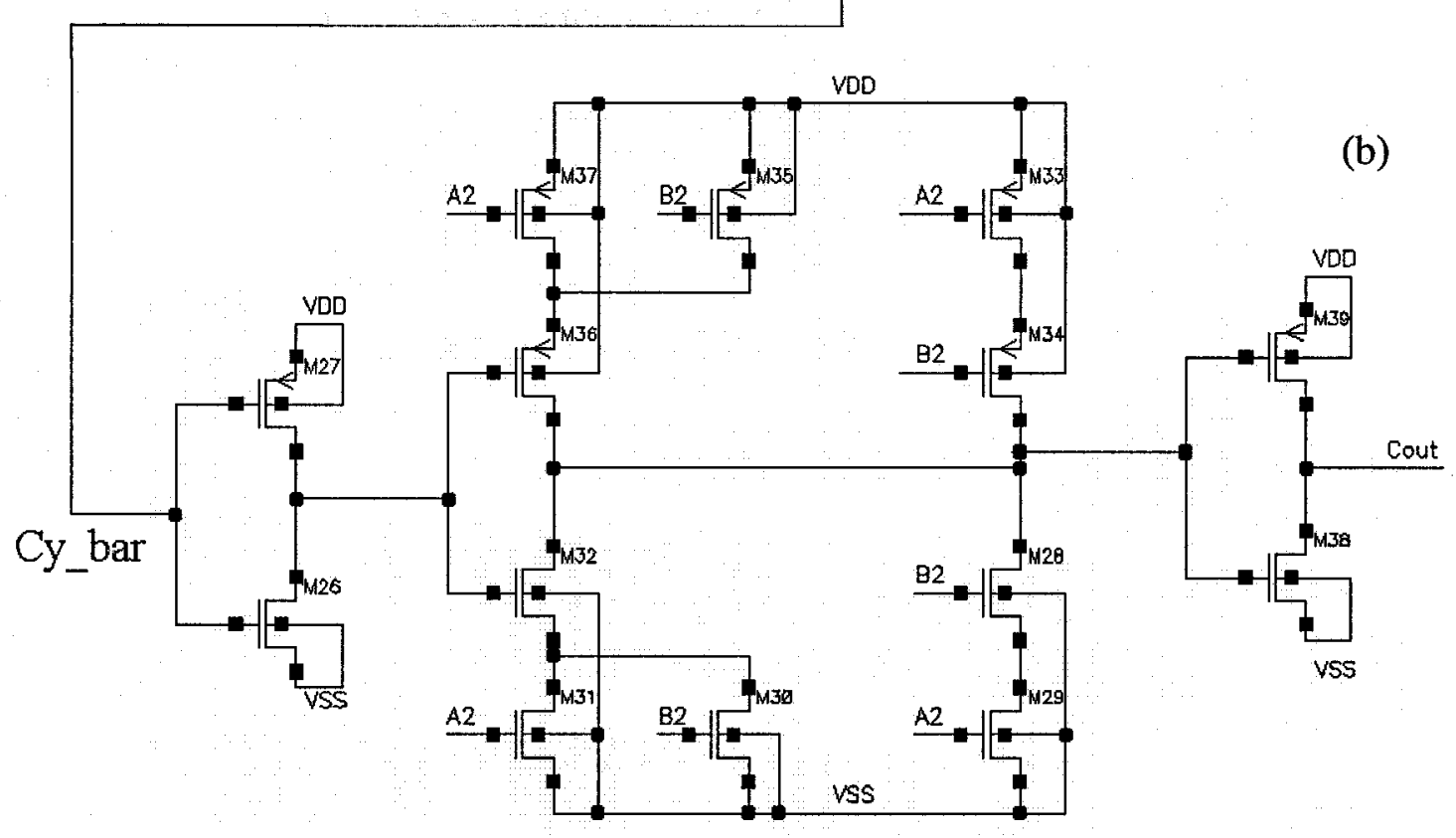

Figure 4.3 Carry circuit of 2-bit full adder before optimization

$A_{1}, A_{2}, B_{1}, B_{2}$ and $C$ in are the full adder's input signals. $C y \_b a r$ is the inverted carry signal produced by the inputs $A_{1}, B_{1}$ and $C i n$. It passes through two inverters (transistors M26, M27 and M38, M39) and a carry generation network (transistors M28 M37). Of the three signals $\operatorname{Sum}_{1}, \operatorname{Sum}_{2}$ and $C_{\text {out }}$, the $C_{\text {out }}$ signal will have the longest delay. The inverting property of the full adder [29],

$$
\overline{C_{\text {out }}}\left(\overline{A_{2}}, \overline{B_{2}}, \overline{C_{y}}\right)=C_{\text {out }}\left(A_{2}, B_{2}, C_{y}\right) \text { where } \overline{C_{y}} \text { is } C y_{-} \text {bar. }
$$


permits a modification to the carry circuit which removes two inverters from the critical path and $\operatorname{Sum}_{I}$ then replaces $C_{\text {out }}$ as the signal having the longest delay. The modified circuit is drawn in Figure 4.4.
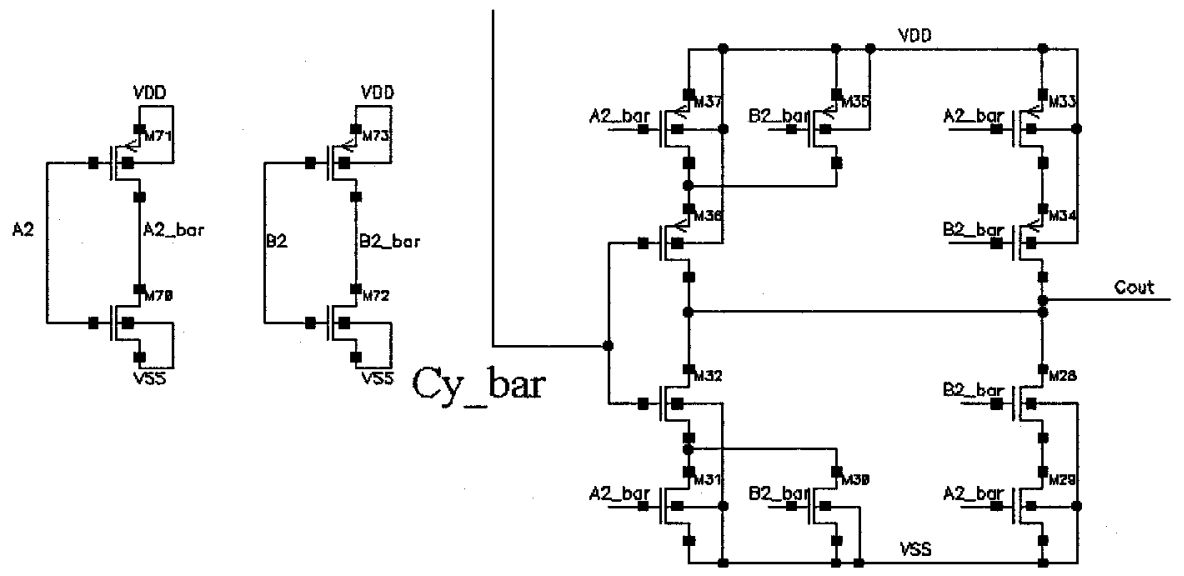

Figure 4.4 Modified carry circuit

Another optimization has been made to the $\mathrm{Sum}_{1}$ circuit, drawn in Figure 3.24 (a). The calculation of $\mathrm{Sum}_{1}$ has two steps:

First, calculate the exclusive-OR of $A_{l}$ with $B_{l}$, that is $A_{1} \oplus B_{1}$. Next, take the exclusive-OR of $A_{1} \oplus B_{1}$ and $C_{i n}$, Sum $m_{1}=\left(A_{1} \oplus B_{1}\right) \oplus C_{i n}$. The critical delay of the resulting 2-bit FA will be about 2 XOR gate propagation delays. The clock cycle time is about 4 XOR gate propagation delays with the clocking scheme used.

The optimization of the Sum $_{1}$ circuit was meant to compensate for the high load capacitance at node $\overline{C_{0}}$ in Figure 3.23.

A 2-bit full adder is not available in the cell library used, so a cascade of two 1-bit full adders was used for comparison purposes. The results are shown in Table 4.2.

TABLE 4.2: Comparison of 2-bit full adders

\begin{tabular}{|l|l|l|l|}
\hline Design & Critical Path & Delay & Power \\
\hline \hline $\begin{array}{l}\text { Proposed 2-bit full Adder (Optimize } \\
\text { both } C_{\text {out }} \text { and Sum } \\
\text { circuit) }\end{array}$ & C $_{\text {in }}$ to Sum 2 & $282.49 \mathrm{ps}$ & $88.74 \mu \mathrm{W}$ \\
\hline $\begin{array}{l}\text { 2-bit full Adder (consists of cas- } \\
\text { caded 1-bit standard cell library FA) }\end{array}$ & C $_{\text {in }}$ to Sum & & $116.46 \mu \mathrm{W}$ \\
\hline
\end{tabular}


In Figure 4.5 is shown the waveforms occurring during the simulations.

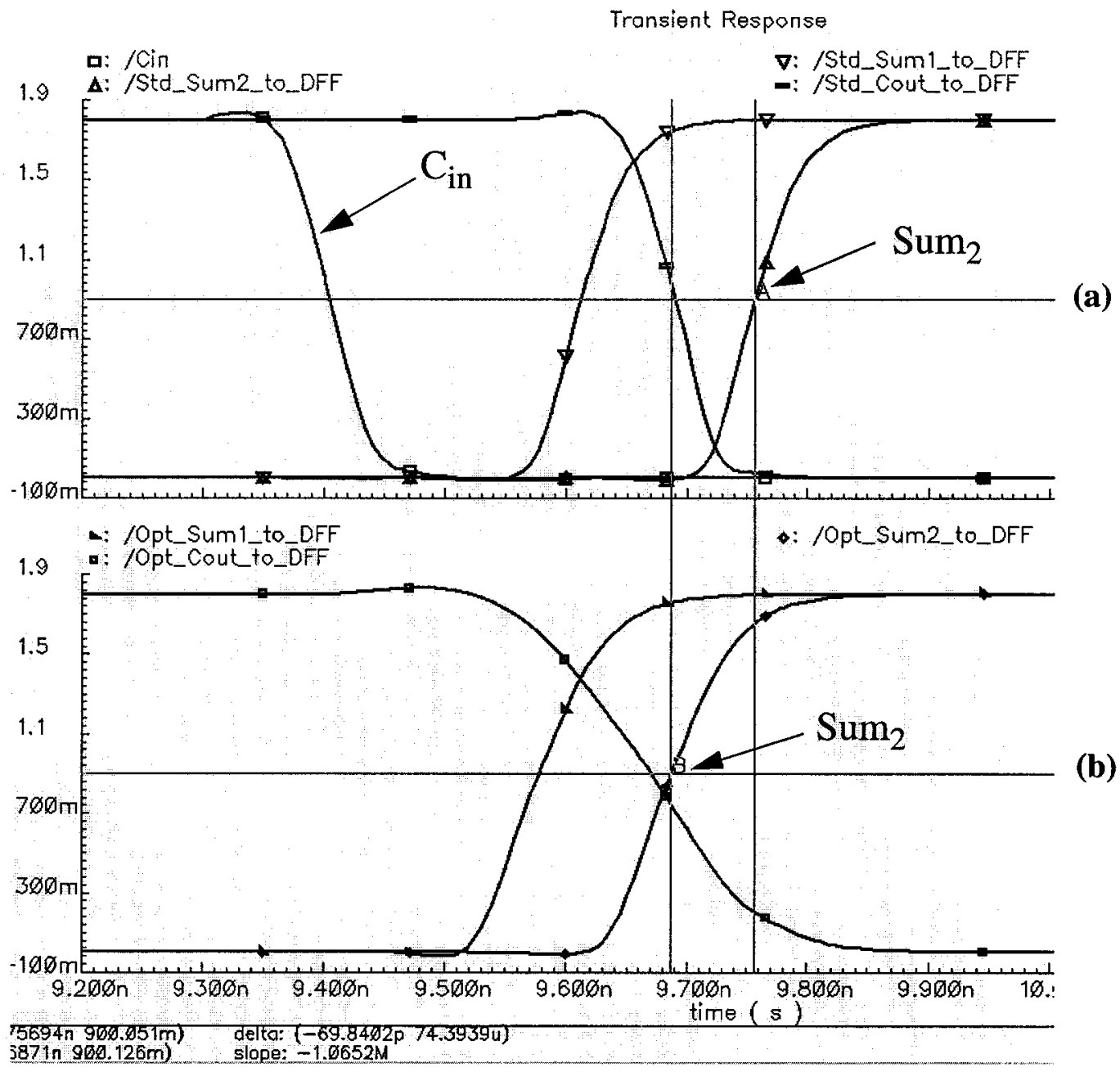

Figure 4.5 Time domain simulation of the 2-bit full adders (a) Standard cell library 1-bit FA cascade and (b) Optimized Adder

The critical delay in both full adder cases is that of the $C_{i n}$ to $\mathrm{Sum}_{2}$ path, marked as $A$ in Figure 4.5 (a) and $B$ in Figure 4.5 (b). The proposed adder is 69.66 ps faster than the library offering, and consumes less power. 


\subsection{Multiplier Simulation}

\subsubsection{Extending Bit Word of Multiplier}

A Montgomery modular multiplier having the desired operand width (number of bits) can be assembled from the necessary number of standard slices enclosed between least significant and most significant slices, with only nearest-neighbor connections, as shown in Figure 4.6. All slices operate from the same global clock signal.

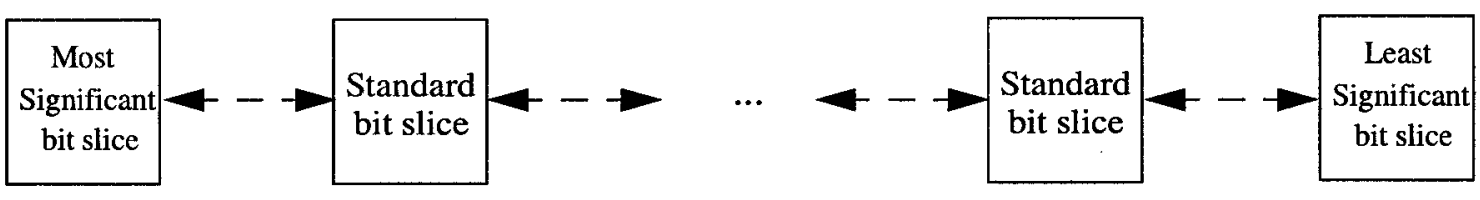

Figure 4.6 Construction of wider word multiplier

To demonstrate this capability, a 128-bit Montgomery modular multiplier was built and a schematic simulation performed. The simulated circuit was found able to operate at $1.15 \mathrm{GHz}$ (excluding $\mathrm{I} / \mathrm{O}$ delays). Device functionality was verified by comparing simulation results to the output of an emulation program written in Visual Basic.

The layout of a 32-bit multiplier has completed and a post-layout simulation performed. It was found able to operate at $1.0 \mathrm{GHz}$. The waveforms observed during a transient simulation are to be found in Appendix B. Layout diagrams of the multiplier and its functional blocks are in Appendix C.

\subsubsection{Multiplier Performance}

The most important performance yardstick of a modular multiplier is its throughput. Also of significance are power consumption, number of clock cycles required for a multiply, ease of extension, and complexity.

The performance of a 32-bit multiplier in post-layout simulation was extrapolated to predict a 1024-bit device's behavior (excluding I/O overhead). Table 4.3 lists the find- 
ings, wherein the worst-case scenario of the RSA processor having one modular multiplier was assumed.

TABLE 4.3: Specifications of a 1024-bit multiplier (Radix-2)

\begin{tabular}{|l|l|l|l|l|l|}
\hline $\begin{array}{l}\text { Supply } \\
\text { voltage }\end{array}$ & $\begin{array}{l}\text { Cycle } \\
\text { time }\end{array}$ & $\begin{array}{l}\text { Number of } \\
\text { clock cycles }\end{array}$ & $\begin{array}{l}\text { Multiplication } \\
\text { time }\end{array}$ & $\begin{array}{l}\text { Throughput of } \\
\text { RSA operation }\end{array}$ & Power \\
\hline \hline $1.8 \mathrm{~V}$ & $1 \mathrm{~ns}$ & $1.25 * 1024$ & $1.28 \mu \mathrm{s}$ & $381.46 \mathrm{k}$ bps & $1.23 \mathrm{~W}$ \\
\hline $1.2 \mathrm{~V}$ & $2 \mathrm{~ns}$ & $1.25 * 1024$ & $2.56 \mu \mathrm{s}$ & $190.73 \mathrm{k} \mathrm{bps}$ & $0.283 \mathrm{~W}$ \\
\hline $1.0 \mathrm{~V}$ & $3 \mathrm{~ns}$ & $1.25 * 1024$ & $3.84 \mu \mathrm{s}$ & $127.15 \mathrm{k} \mathrm{bps}$ & $0.109 \mathrm{~W}$ \\
\hline
\end{tabular}

A corresponding determination, using simulation results appropriate to a 16-bit radix -4 multiplier to predict the capabilities of a 1024-bit radix-4 multiplier, and the findings are listed in Table 4.4. The radix-4 multiplier was designed using the Complementary Pass Logic (CPL) style and was not optimized for power consumption (which was not measured.)

TABLE 4.4: Specifications of a 1024-bit multiplier (Radix-4)

\begin{tabular}{|l|l|l|l|l|}
\hline $\begin{array}{l}\text { Supply } \\
\text { voltage }\end{array}$ & $\begin{array}{l}\text { Cycle } \\
\text { time }\end{array}$ & $\begin{array}{l}\text { Number of } \\
\text { clock cycles }\end{array}$ & $\begin{array}{l}\text { Multiplication } \\
\text { time }\end{array}$ & $\begin{array}{l}\text { Throughput of } \\
\text { RSA operation }\end{array}$ \\
\hline \hline $1.8 \mathrm{~V}$ & $1 \mathrm{~ns}$ & $0.75 * 1024$ & $0.768 \mu \mathrm{s}$ & $635.78 \mathrm{k}$ bps \\
\hline
\end{tabular}

\subsection{Comparison with Published Results}

Comparisons of published radix-2 architectures appear in this section which are summarized in Table 4.5 .

\subsubsection{Number of Clock Cycles and Maximum Clocking Speed}

The present design uses $1.25 \mathrm{~N}$ clock cycles to perform an $\mathrm{N}$-bit modular multiplication; the length of one clock cycle is about 4 XOR gate propagation delays. Its critical path delay is $1.7 \mathrm{~ns}$ (schematic) when realized with a $0.35 \mu \mathrm{m}$ CMOS process and $1 \mathrm{~ns}$ (extracted) with a $0.18 \mu \mathrm{m}$ CMOS process. 


\subsubsection{Number of Transistors}

The fully custom design requires fewer transistors than do the Montgomery modular multipliers reported beforehand. This design uses 142 transistors per bit, to be compared with a minimum figure of 172 needed in the published designs.

\subsubsection{Power Consumption}

This design can work from a standard $1.8 \mathrm{~V}$ supply, and even as low as $1.0 \mathrm{~V}$.

Power consumption values at three supply voltages are listed in Table 4.3. The lowest figure, occurring with $\mathrm{V}_{\text {supply }}=1.0 \mathrm{~V}$, is only $109 \mathrm{~mW}$ (clock cycle time $=3 \mathrm{~ns}$ ).

Since there seems to be no literature data for multipliers realized in $0.18 \mu \mathrm{m}$ CMOS technology, an 8-bit version of the radix-2 architecture of this thesis was implemented in a $0.35 \mu \mathrm{m}$ CMOS process to facilitate comparison of its behavior with that of published designs. Table 4.5 shows that the critical path delay is $1.7 \mathrm{~ns}$.

TABLE 4.5: Design comparisons in $0.35 \mu \mathrm{m}$ technology

\begin{tabular}{|l|l|l|l|l|}
\hline Design & Critical path & $\begin{array}{l}\text { Cycle } \\
\text { time }\end{array}$ & $\begin{array}{l}\text { Number of } \\
\text { clock cycles }\end{array}$ & $\begin{array}{l}\text { Multiplication } \\
\text { time }\end{array}$ \\
\hline \hline Walter [20] & $4 \mathrm{~T}_{\mathrm{AND} / \mathrm{OR}}+\mathrm{T}_{\mathrm{XOR}}$ & $2.4 \mathrm{~ns}[25]$ & $\sim 2 * 1024[25]$ & $4.9 \mu \mathrm{s}[25]$ \\
\hline Kornerup[24] & $\mathrm{T}_{\mathrm{AND} / \mathrm{OR}}+4 \mathrm{~T}_{\mathrm{XOR}}$ & $3.6 \mathrm{~ns}[25]$ & $\sim 2 * 1024[25]$ & $7.4 \mu \mathrm{s}[25]$ \\
\hline Chen[22] & $\mathrm{T}_{\mathrm{AND} / \mathrm{OR}^{+}+2 \mathrm{~T}_{\mathrm{XOR}}}$ & $2.0 \mathrm{~ns}[25]$ & $\sim 4 * 1024[25]$ & $8.2 \mu \mathrm{s}[25]$ \\
\hline Tsai (Double-layer)[25] & $\mathrm{T}_{\mathrm{AND} / \mathrm{OR}+2 \mathrm{~T}_{\mathrm{XOR}}}$ & $2.0 \mathrm{~ns}$ & $\sim 2 * 1024$ & $4.1 \mu \mathrm{s}$ \\
\hline Tsai (Non-interlaced)[25] & $\mathrm{T}_{\mathrm{AND} / \mathrm{OR}}+3 \mathrm{~T}_{\mathrm{XOR}}$ & $2.7 \mathrm{~ns}$ & $\sim 1 * 1024$ & $2.8 \mu \mathrm{s}$ \\
\hline This design (schematic) & $4 \mathrm{~T}_{\mathrm{XOR}}$ & $1.7 \mathrm{~ns}$ & $1.25 * 1024$ & $2.2 \mu \mathrm{s}$ \\
\hline
\end{tabular}

$\mathrm{T}_{\mathrm{AND} / \mathrm{OR}}$ : Delay time of an AND/OR gate.

$\mathrm{T}_{\mathrm{XOR}}$ : Delay time of an XOR gate.

Table 4.6 lists the hardware resource requirements and power consumption of different designs. This design is seen to have the lowest transistor count achieved by a new architecture. The power consumption of this design is higher than that of Walter [20], Kornerup [24] and Tsai (Non-interlaced) [25] (19\%, 71\%, 12\% respectively). However, the 
performance of this design (multiplication time, shown in Table 4.5) is better than these designs.

The higher power consumption is due to the large transistors used in the external pulse generators.

TABLE 4.6: Comparison of area and transistor count

\begin{tabular}{|l|l|l|l|}
\hline & & Transistor & Power \\
Design & Gates/bit & Count/bit & (W) \\
\hline \hline Walter [20] & 9AND/OR +5XOR +5DFF & $240[25]$ & $0.557[25]$ \\
\hline Kornerup [24] & 2AND/OR +2FA+3DFF & $172[25]$ & $0.387[25]$ \\
\hline Chen [22] & 2AND/OR +2FA+7DFF & $240[25]$ & $0.735[25]$ \\
\hline Tsai (Double-layer) [25] & 6AND/OR +4XOR+6DFF & 240 & 0.681 \\
\hline Tsai (Non-interlaced) [25] & 12.5AND/OR +6XOR+3.5DFF & 209 & 0.593 \\
\hline This design (radix-2) & 2FA+1MUX4-1+2.5DFF ${ }^{2}$ & $142^{3}$ & 0.663 \\
\hline
\end{tabular}

1. Simulated at $278 \mathrm{MHz}$ in CMOS $0.35 \mu \mathrm{m}$ process.

2. Shift register and "Stop" circuitry are excluded in order to facilitate comparison with other designs.

3. Based on the full custom design approach.

\subsection{Summary}

This design is superior to existing ones - faster, smaller transistor count, wide supply range and acceptable power consumption. Its ability to work over a range of supply voltages gives it the flexibility to operate in systems with a wide range of power consumption and speed requirements. 


\subsection{Conclusion}

Two high-speed modular multiplication circuit architectures based on Montgomery's algorithm have been designed, each employing a novel clocking scheme. Custom, performance optimized building blocks were used, ones showing significant improvements over standard cell library units. The device is versatile in that a range of supply voltages is possible and power consumption is always acceptable.

Two architectural improvements have also been made:

1. A new pipeline approach significantly reduces the number of clock cycles taken by a single modular multiplication.

2. Pre-computation circuits result in a critical path involving the delays of only four XOR gates.

\subsection{Future Work}

A natural extension to the present work would involve implementing a control unit and thence an entire RSA processor, possibly taking advantage of the radix -4 option described in this work to get extra speed [28].

As CMOS technology scales down into the 90 and 65 nanometer, the power lost due to leakage becomes a nonnegligible component of total power consumption. Techniques to minimize leakage current, such as increasing length of transistors, should be applied. 


\section{Bibliography}

1. A. Bhimani, Securing the commercial internet, Communications of the ACM, vol 39, no. 6, pp. 29-35. June 1996.

2. William Stallings, Cryptography and network Security: Principles and Practice, 2nd ed., Prentice-Hall, 1999.

3. Whitfield Diffie and Martin E. Hellman, New Direction in Cryptography, IEEE Trans. on Information Theory, pp. 644-654, vol. it-22, no. 6, Nov. 1976.

4. R. L. Rivest, A. Shamir, and L. Adleman, A Method for Obtaining Digital Signatures and Public-key Cryptosystems, Communications of the ACM, pp. 120-126, vol. 21, no. 2, Feb. 1978.

5. Bruce Schneier, Applied Cryptography Protocols, Algorithms, and Source Code in C, 2nd Ed., John Wiley \& Sons, Inc., 1996.

6. Alfred J. Menezes, Paul C. van Oorschot and Scott A. Vanstone, Handbook of Applied Cryptography, CRC Press, 1996.

7. Darrel Hankerson, Alfred Menezes and Scott Vanstone, Guide to Elliptic Curve Cryptography. Springer, 2004.

8. Data Encryption Standard. National Bureau of standards, U.S. Department of Commerce, Federal Information Processing Standards Publications. http:// www.itl.nist.gov/fipspubs/fip46-2.htm.

9. http://csrc.nist.gov/publications/fips/05-9945-DES-Withdrawl.pdf

10. Friedrich L. Bauer, Decrypted Secrets: Methods and Maxims of Cryptology, 2nd Ed., Springer-Verlag, 2000.

11. T. El Gamal, A public key cryptosystem and signature scheme based on discrete logarithms. IEEE Trans. on Information Theory. pp. 469-472, vol. IT-31, no. 4 July 1985.

12. Proposed Federal Information Processing Standard for Digital Signature Standard, Federal Register, vol. 56, no. 169, pp. 42980-42982, 30 Aug. 1991. 
13. Arto Salomaa, Public-Key Cryptography, Springer-Verlag, Berlin, 1990.

14. Donald E. Knuth, The Art of Computer Programming, vol. 2, Seminumerical Algorithms, 3rd Ed., Addison-Wesley, 1997.

15. http://www.rsasecurity.com/rsalabs/node.asp?id=2964.

16. Young Sae Kim, Woo Seok Kang, and Jun Rim Choi, Implementation of 1024-bit Modular Processor for RSA Cryptosystem. The Second IEEE Asia Pacific Conference on ASIC, pp. 187-190. Aug. 2000.

17. Peter L. Montgomery, Speeding the Pollard and elliptic curve methods of factorization. Mathematics of Computation, 48(177) pp. 243-264, Jan. 1987.

18. Marc Joye and Sung-Ming Yen, The Montgomery Powering Ladder, Cryptographic Hardware and Embedded System (CHES' 02), vol. 2523 of Lecture Notes in computer science, Springer-Verlag, pp. 291-302, 2003.

19. Miloš D. Ercegovac, Tomás Lang, Digital Systems and Hardware/Firmware Algorithms, John Wiley \& Sons, Inc. 1985.

20. C. D. Walter, Systolic modular multiplication, IEEE Trans. Comput., vol. 42, no. 3, pp. 368-378, Mar. 1993.

21. Keon-Jik Lee, Kee-Young Yoo, Systolic multiplier for Montgomery's algorithm, VLSI Journal Integration, pp. 99-109, vol. 32, 2002.

22. Po-Song Chen, Shih-Arn Hwang, and Cheng-Wen Wu, A Systolic RSA Public key Cryptosystem, Proc. ISCAS, vol.4, pp. 408-411, 1996.

23. Ching-Chao Yang, Tian-Sheuan Chang, and Chein-Wei Jen, A New RSA Cryptosystem Hardware Design Based on Montgomery's Algorithm, IEEE Trans. Circuits and Systems-II: Analog and Digital Signal Processing, vol. 45, no. 7, pp. 908-913, July 1998.

24. P. Kornerup, A systolic, Linear-Array Multiplier for a Class of Right-Shift Algorithms, IEEE Trans. Computers, vol. 43, no. 8, pp. 892-898, Aug. 1994.

25. Wei-Chang Tsai, C. Bernard Shung, and Sheng-Jyh Wang, Two Systolic Archituctures for Modular Multiplication, IEEE Trans. on VLSI Systems, vol. 8, no. 1, pp. 103-107, Feb. 2000. 
26. Peter L. Montgomery, Modular Multiplication Without Trial Division, Mathematics of Computation, vol. 44, no. 170, pp. 519-521, Apr. 1985.

27. Nadia Nedjah and Luiza de Macedo Mourelle, Reconfigurable Hardware Implementation of Montgomery Modular Multiplication and Parallel Binary Exponentiation, Euromicro Symposium on Digital System Design, pp.226-233, Sept. 2002.

28. Holger Orup, Simplifying Quotient Determination in High-Radix Modular Multiplication, Proc. 12th Symp. on Computer Arithmetic, pp. 193-199, July 1995.

29. Jan M. Rabaey, Anantha Chandrakasan, and Borivoje Nikolić, Digital Integrated Circuits: A design Perspective, 2nd Ed., Prentice Hall, 2003.

30. Reto Zimmermann and Wolfgang Fichtner, Low-Power Logic Styles: CMOS Versus Pass-Transistor Logic. IEEE J. Solid-State Circuits, vol. 32, no. 7, pp. 1079-1090, July 1997.

31. S. -Y. Kung, VLSI Array Processors, Englewood Cliffs, NJ: Prentice Hall, 1988.

32. Neil H. E. Weste and Kamran Eshraghian, Principles of CMOS VLSI Design, 2nd Ed., Addison Wesley Longman, 1992.

33. Vladimir Stojanovic and Vojin G. Oklobdzija, Comparative Analysis of Master-Slave Latches and Flip-Flops for High-Performance and Low-Power Systems, IEEE J. Solid-State Circuits, vol. 34, no. 4, pp. 536-548, Apr. 1999.

34. J. Tschanz, S. Narendra, Z. P. Chen, S. Borkar, M. Sachdev, and V. De, Comparative delay and energy of single edge-triggered \& dual edge-triggered pulsed flip-flops for high-performance microprocessors. in Proc. ISPLED'01, Huntington Beach, CA, pp. 147-152, Aug. 2001.

35. David A. Hodges, H. G. Jackson and R. A. Saleh, Analysis and Design of Digital Integrated Circuits, 3rd Ed., McGraw-Hill, 2003.

36. Peiyi Zhao, Tarek K. Darwish and Magdy A. Bayoumi, High-Performance and LowPower Conditional Discharge Flip-Flop, IEEE Trans. on Very Large Scale Integration System, vol. 12, no. 5, pp. 477-484, May 2004.

37. Gary Yeap, Practical Low Power Digital VLSI Design, Kluwer Academic Publishers, Norwell, 1998. 


\section{Appendix A: Self-duality Property of the Adder Functions}

The equations for a 1-bit full adder include the Sum and Carry functions.

The Carry function is

$$
\text { Carry }=A B+B C_{i n}+A C_{i n}
$$

which may be transformed to:

$$
\text { Zarry }=A B+C_{i n} \cdot(A+B)=(A+B) \cdot\left(C_{i n}+A B\right)
$$

If $A B+C_{i n} \cdot(A+B)$ describes the NMOS pull down network (PDN) of a CMOS gate and $(A+B) \cdot\left(C_{i n} \cdot A B\right)$ its PMOS pull up network (PUN), then the Carry function will have a symmetrical architecture.

Similarly, in the case of the Sum function,

$$
u m=A B C_{i n}+\overline{C_{o}} \cdot\left(A+B+C_{i n}\right)=\left(A+B+C_{i n}\right) \cdot\left(\overline{C_{o}}+A B C_{i n},\right.
$$

if $4 B C_{i n}+\bar{C}_{o} \cdot\left(A+B+C_{i n}\right)$ is used to implement the adder's PDN, and $\left.A+B+C_{i n}\right) \cdot\left(\overline{C_{o}}+A B C_{i n}\right)$ its PUN, a symmetrical architecture will likewise result. 


\section{Appendix B: 32-bit Modular Multiplier Transient Simulation}

The simulation takes three inputs, the numbers to be multiplied ( $A$ and $B$ ), and the modulus $(M)$. The values used in this simulation were:

$$
\begin{aligned}
& A=(6 \mathrm{AB} 5,6 \mathrm{~A} 76)_{\mathrm{HEX}} \\
& B=(7154,566 \mathrm{~B})_{\mathrm{HEX}} \\
& M=(3 \mathrm{~A} 50,5 \mathrm{EAD})_{\mathrm{HEX}}
\end{aligned}
$$

The result obtained using these numbers, is:

$R=0011,1110,0010,1001,0111,0101,1111,0101$ 
Simulations were done for supply voltage of 1.8V (Figure B-1) and 1.0V (Figure B-2). The signals occurring are plotted below.

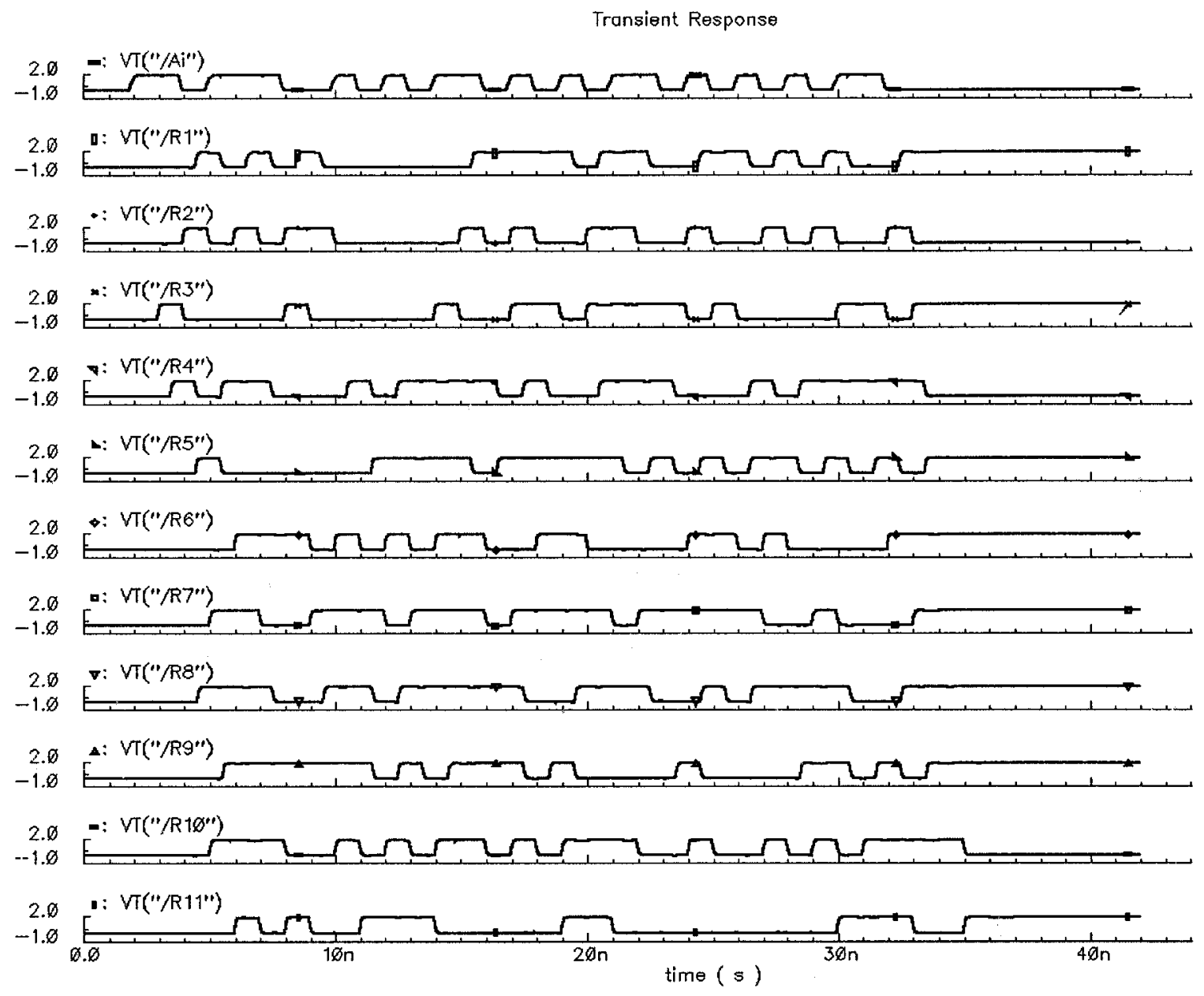

Figure B-1 (a) 1.8 V Supply voltage transient simulation 
Transient Response

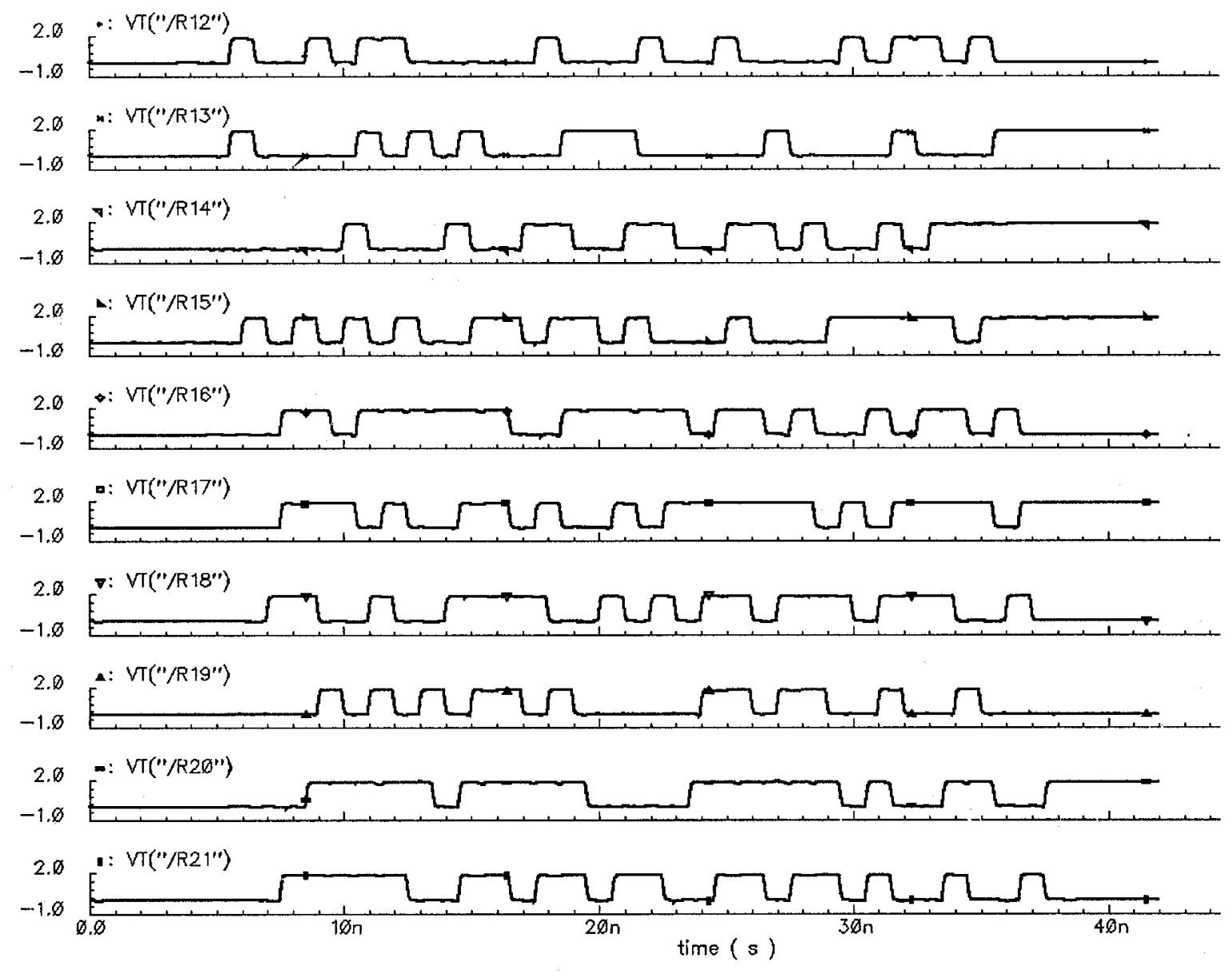

Figure B-1 (b) 1.8 V Supply voltage transient simulation 


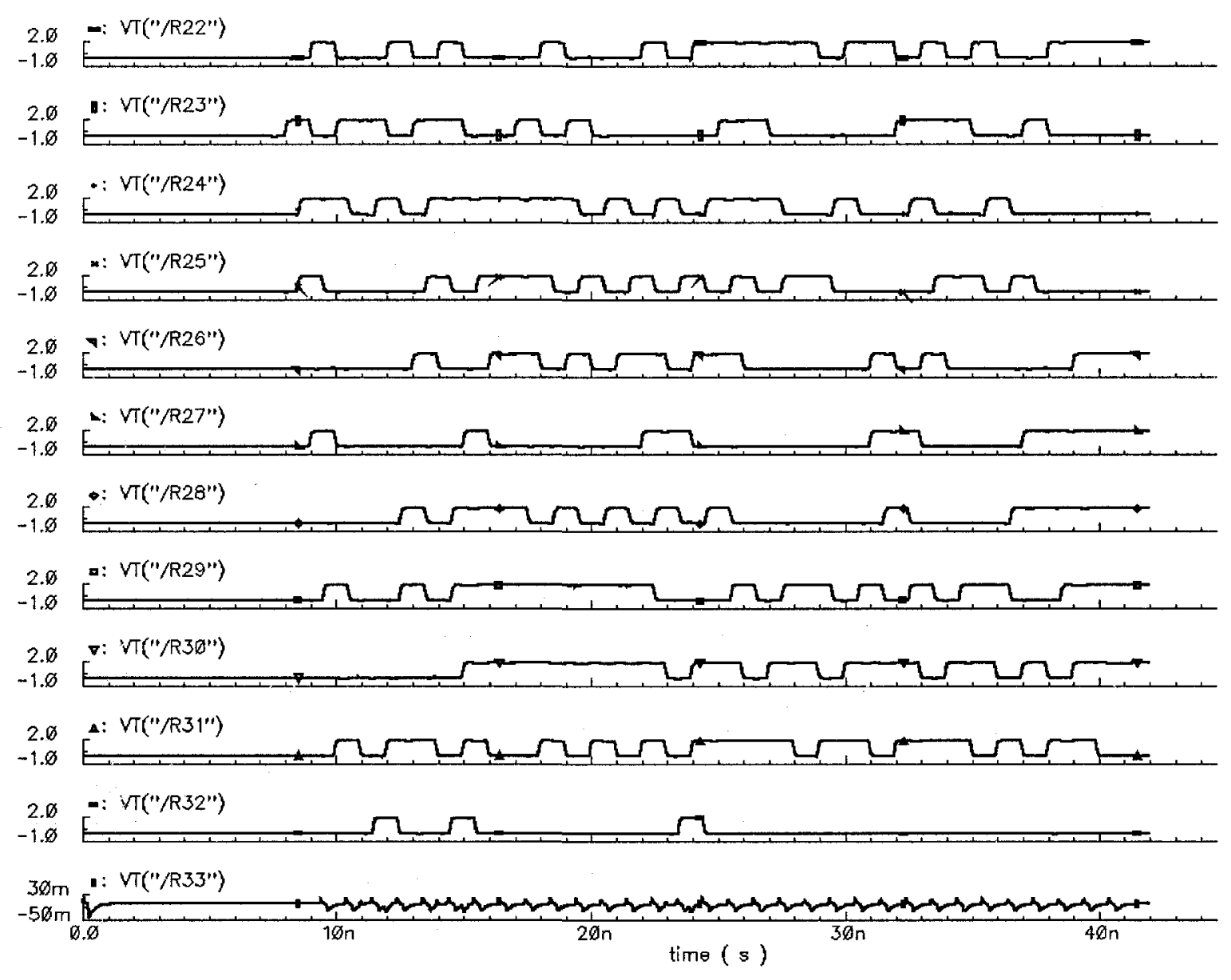

Figure B-1 (c) 1.8 V Supply voltage transient simulation 


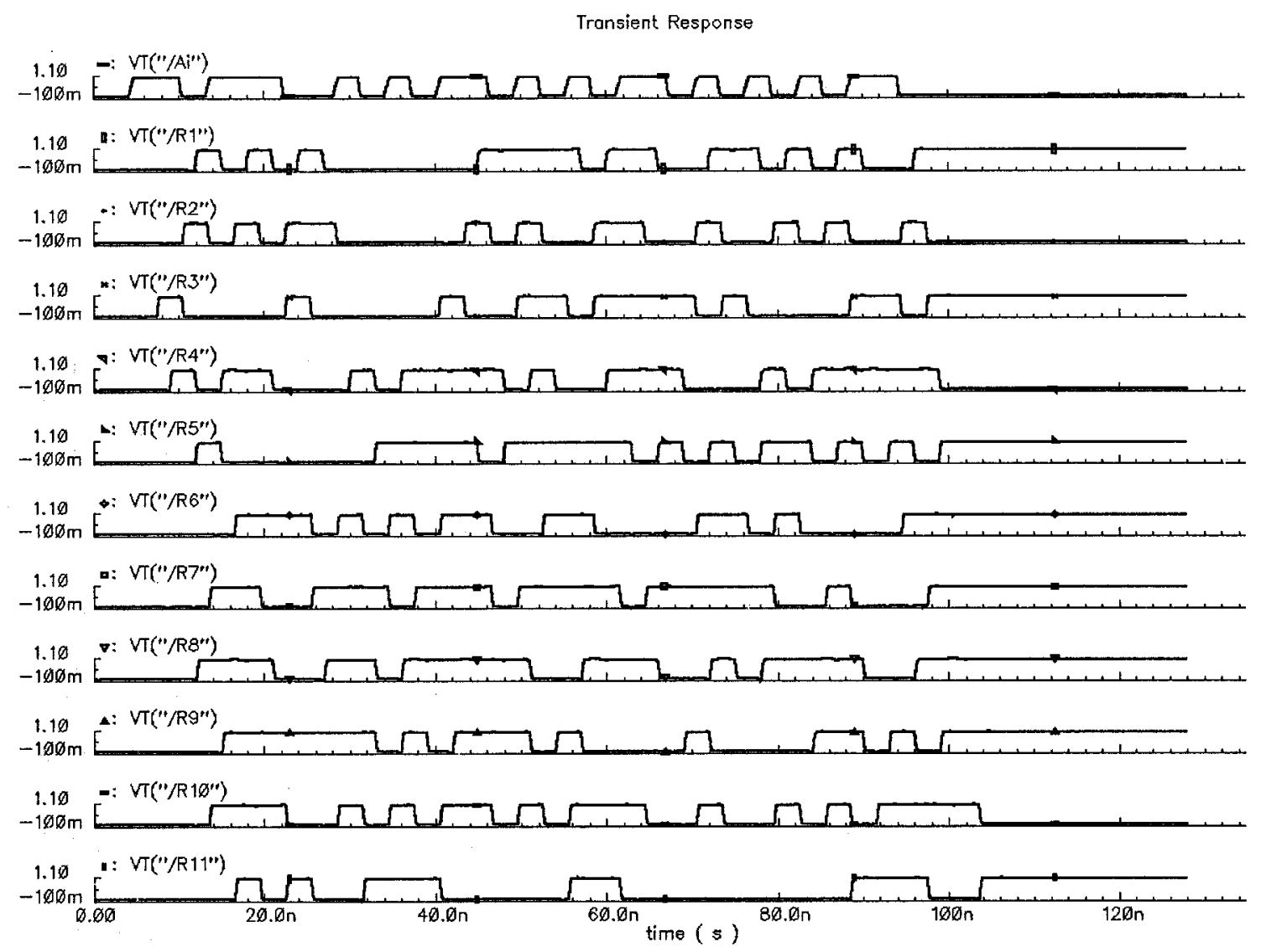

Figure B-2 (a) 1.0 V Supply voltage transient simulation 


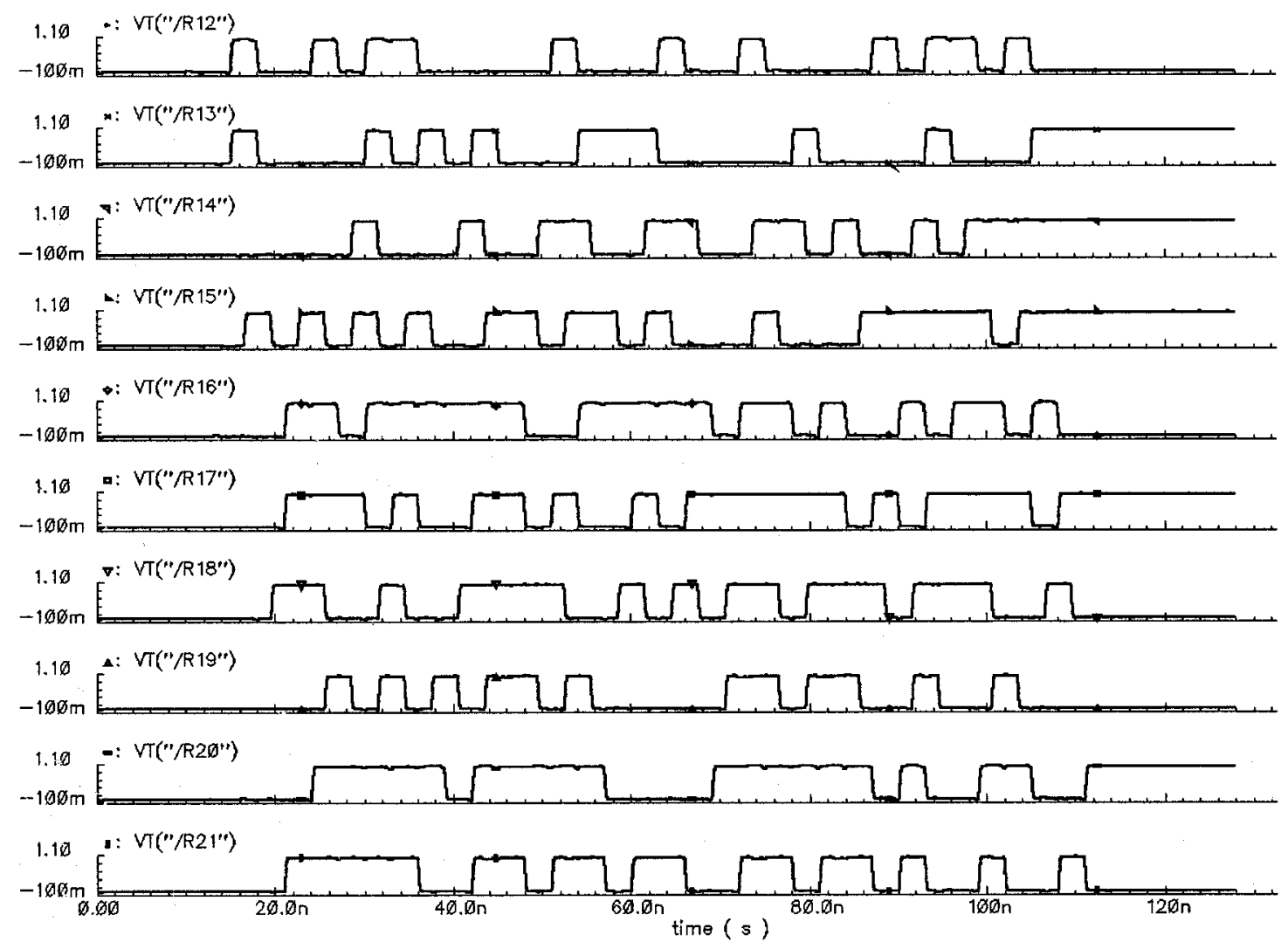

Figure B-2 (b) 1.0 V Supply voltage transient simulation 
Tronsient Response

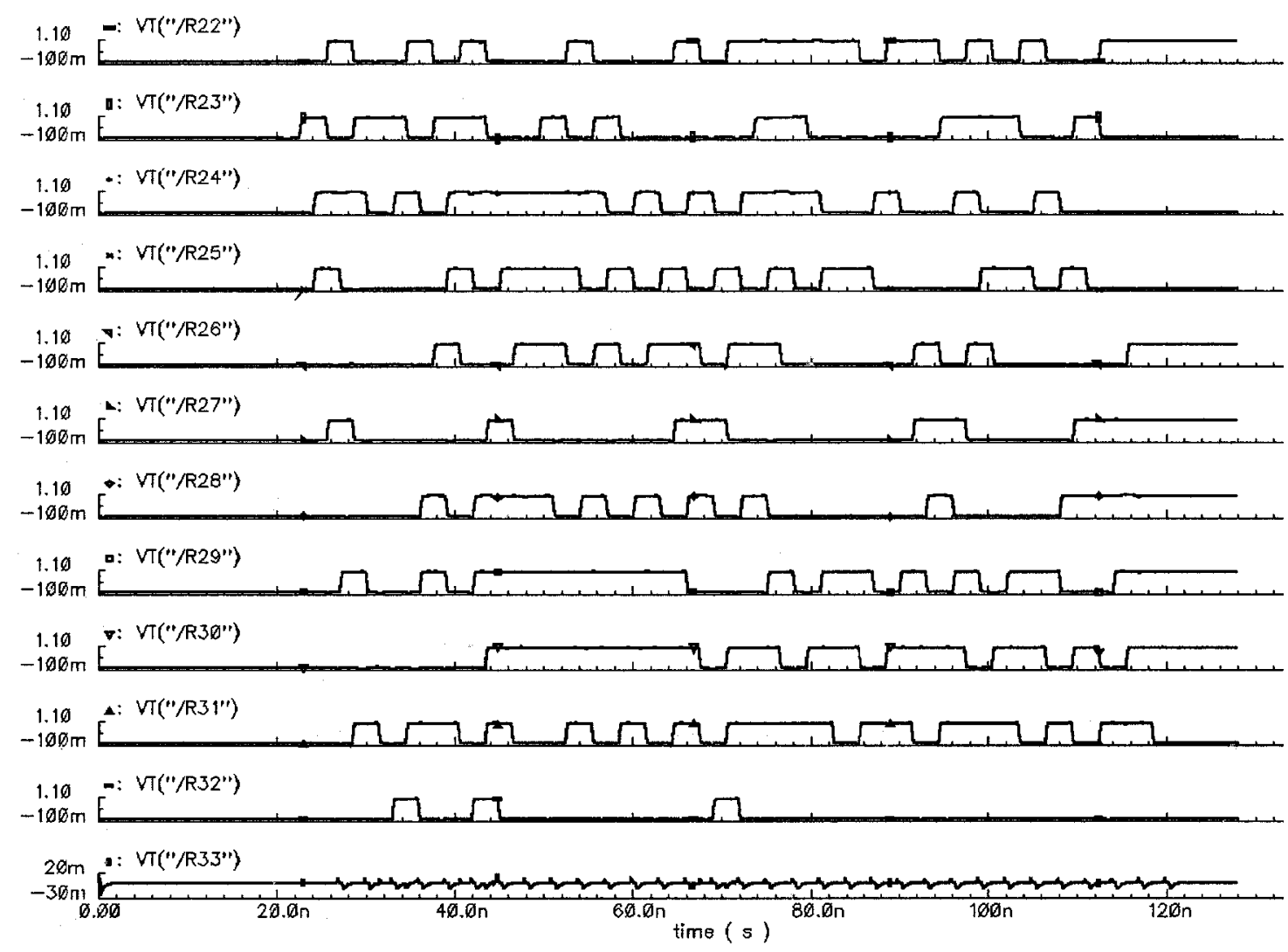

Figure B-2 (c) 1.0 V Supply voltage transient simulation 


\section{Appendix C: Layout Diagrams of Multiplier Functional Blocks}

The layouts for the important parts of a 32-bit Montgomery modular multiplier are presented in this Appendix.
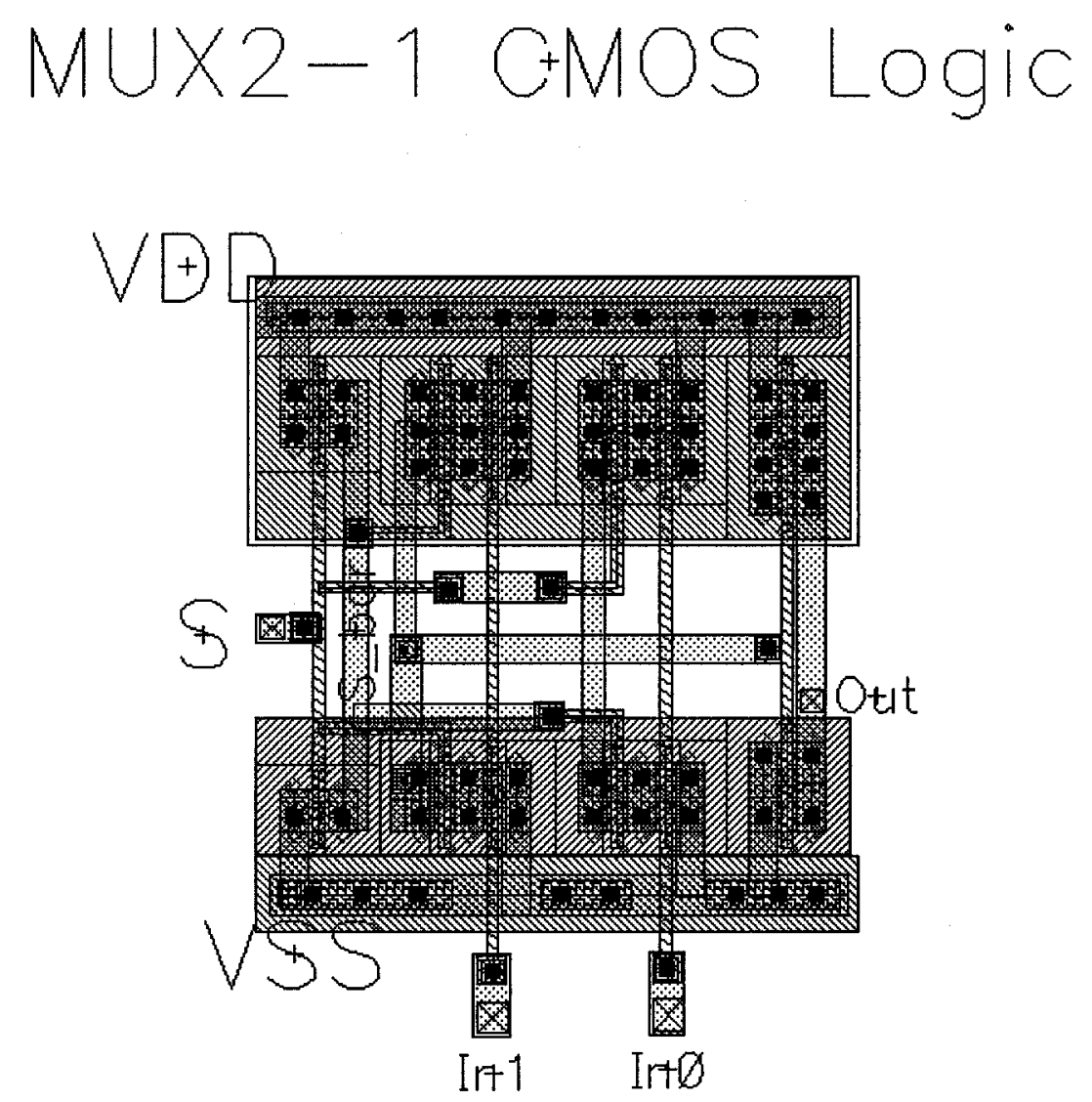

Figure C-1 Multiplexer 2-1 (MUX2-1) 


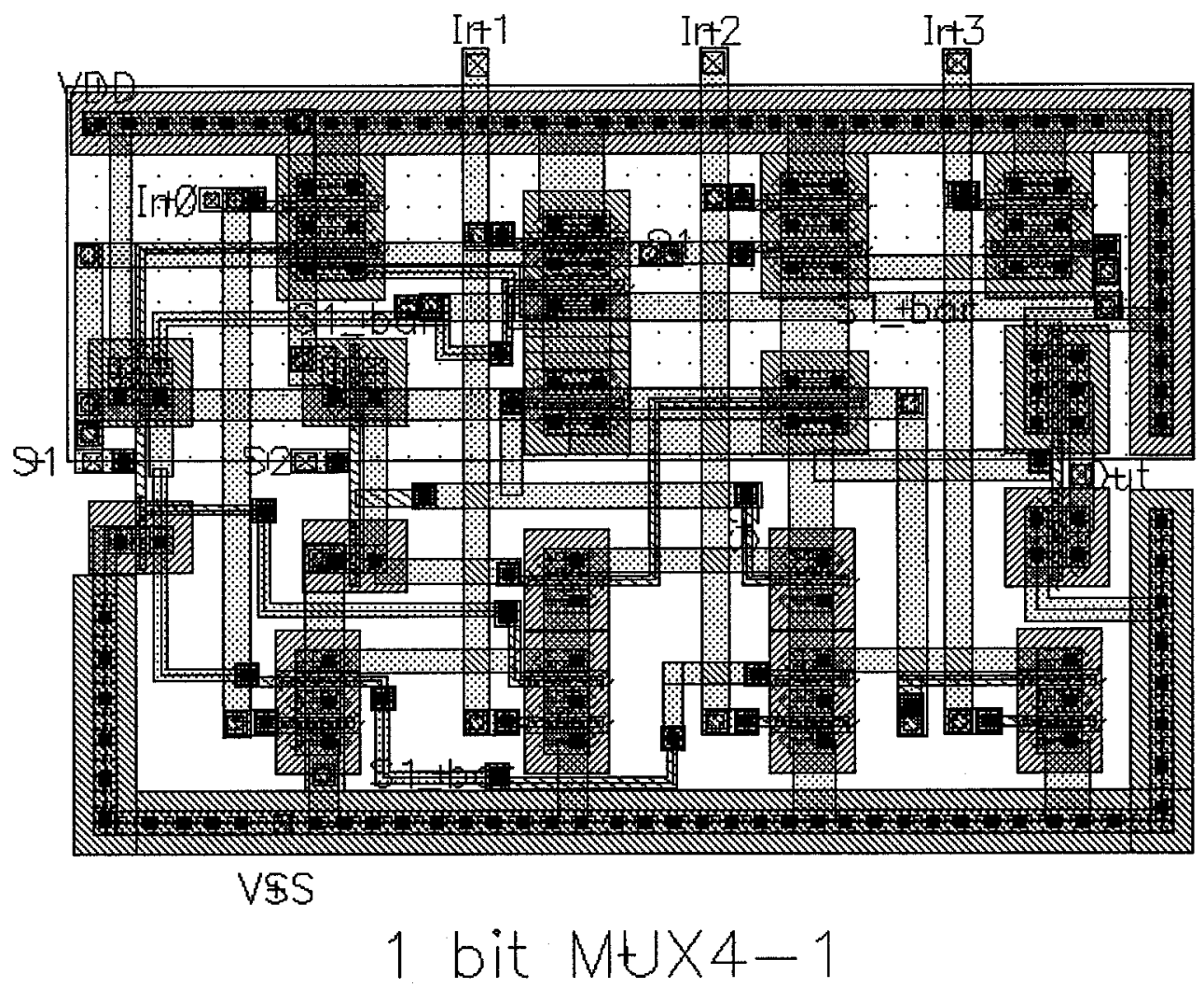

Figure C-2 Multiplexer 4-1 (Mux4-1) 


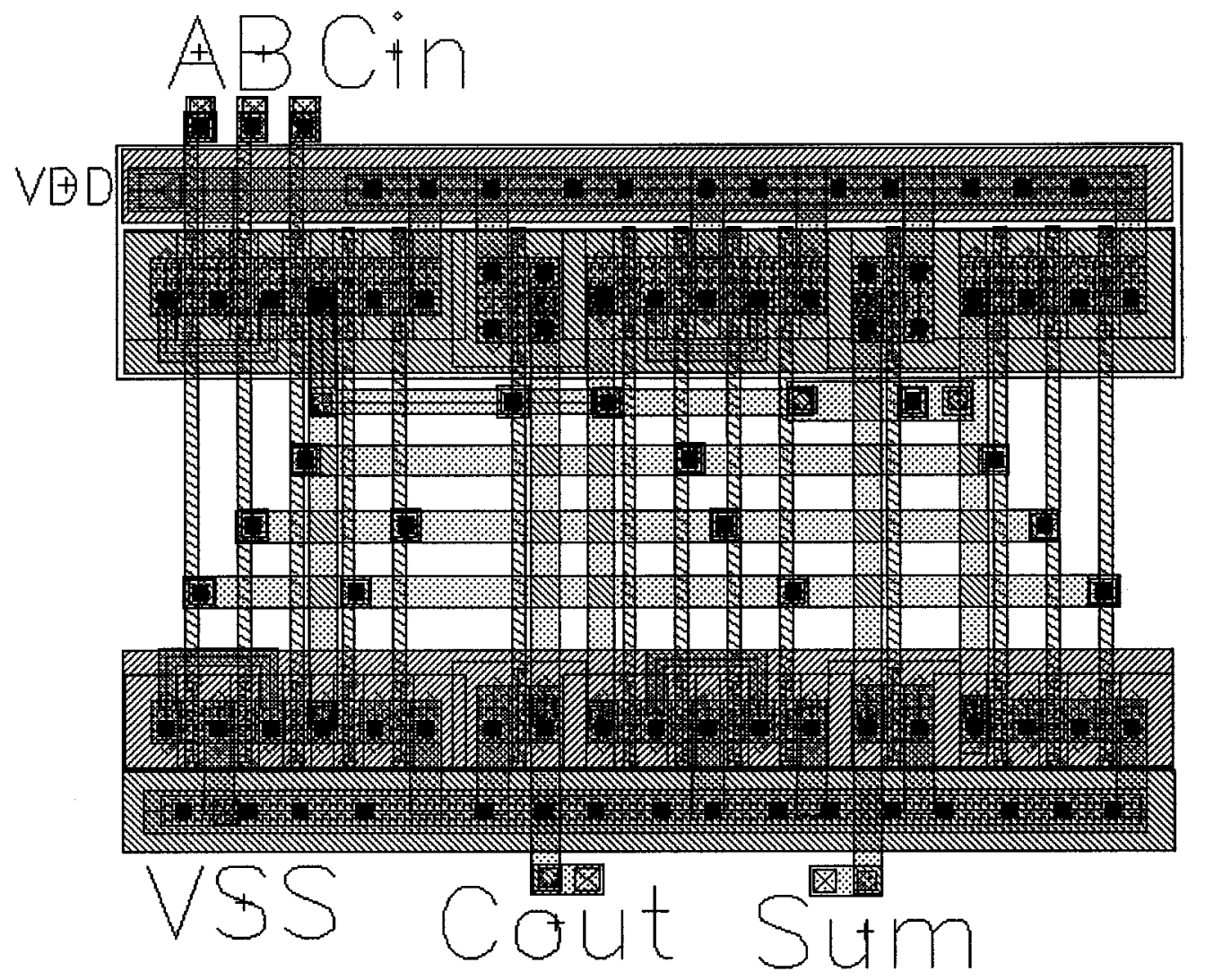

Figure C-3 1-bit full adder 


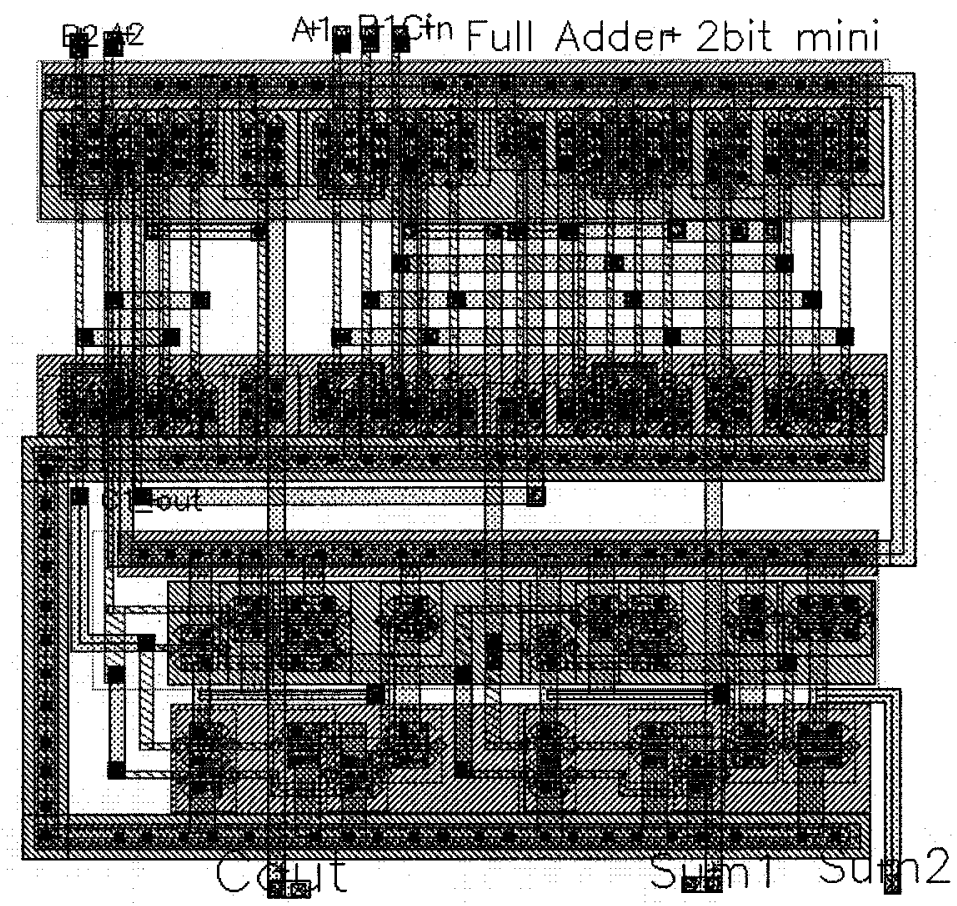

Figure C-4 2-bit full adder 


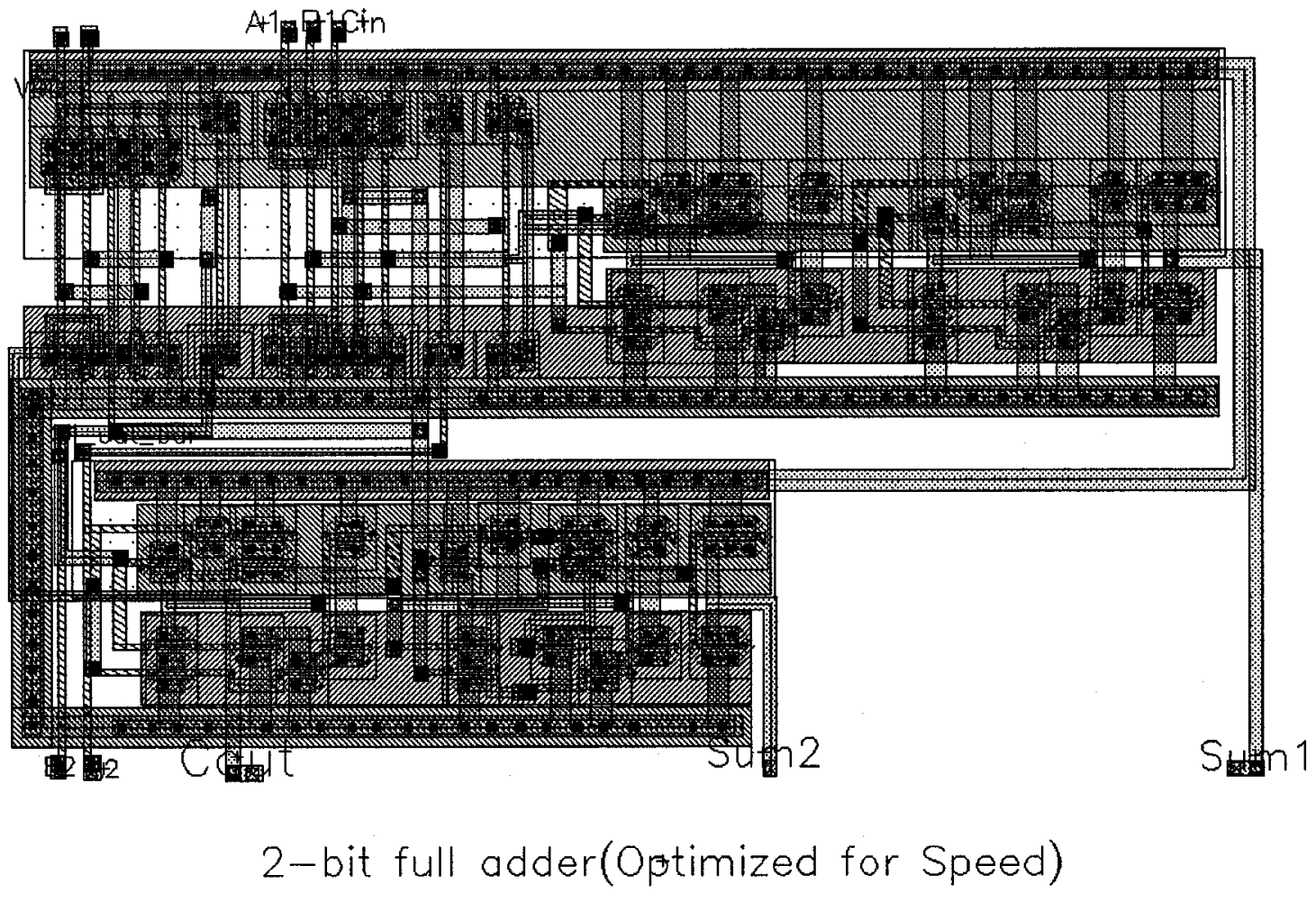

Figure C-5 Optimized 2-bit full adder 


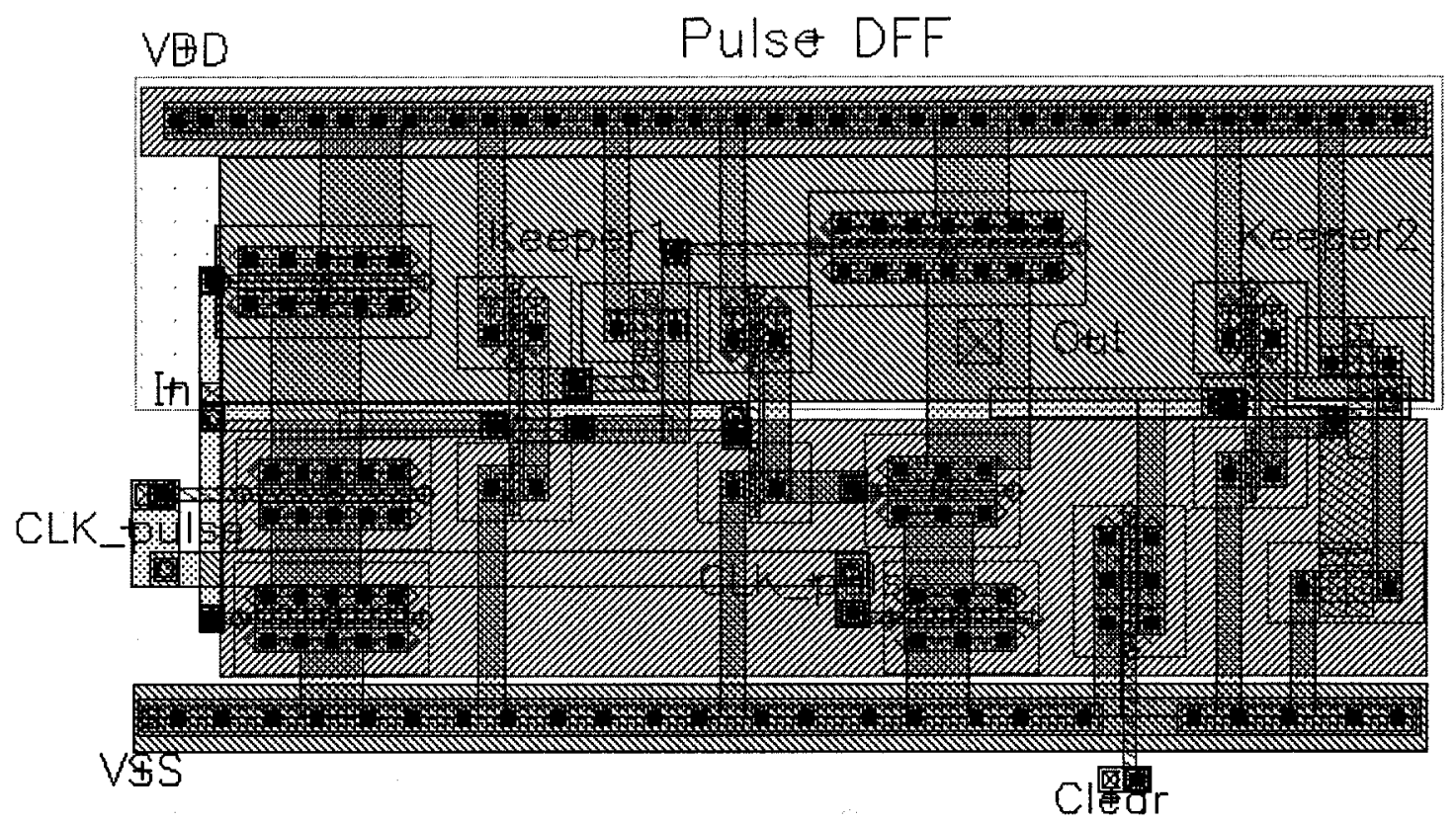

Figure C-6 pulsed D flip flop 


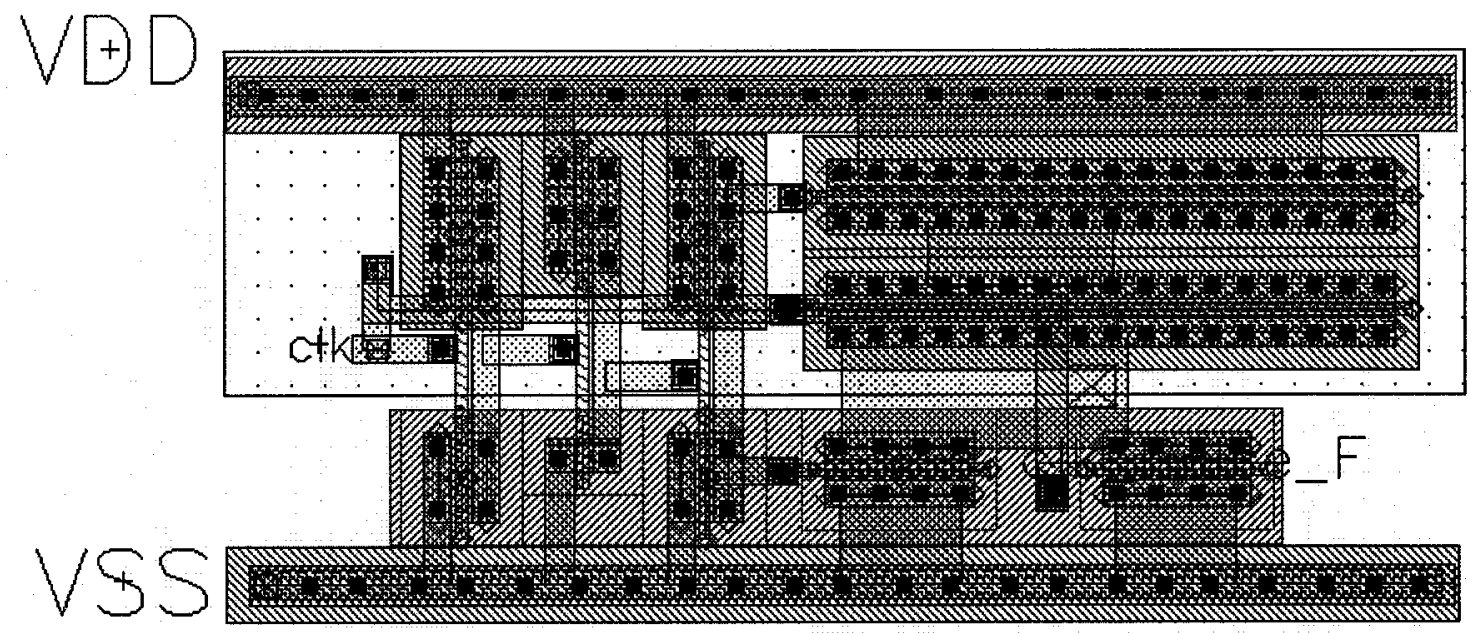

Figure C-7 Pulse generator for D flip flop (fall edge) 


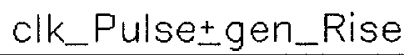

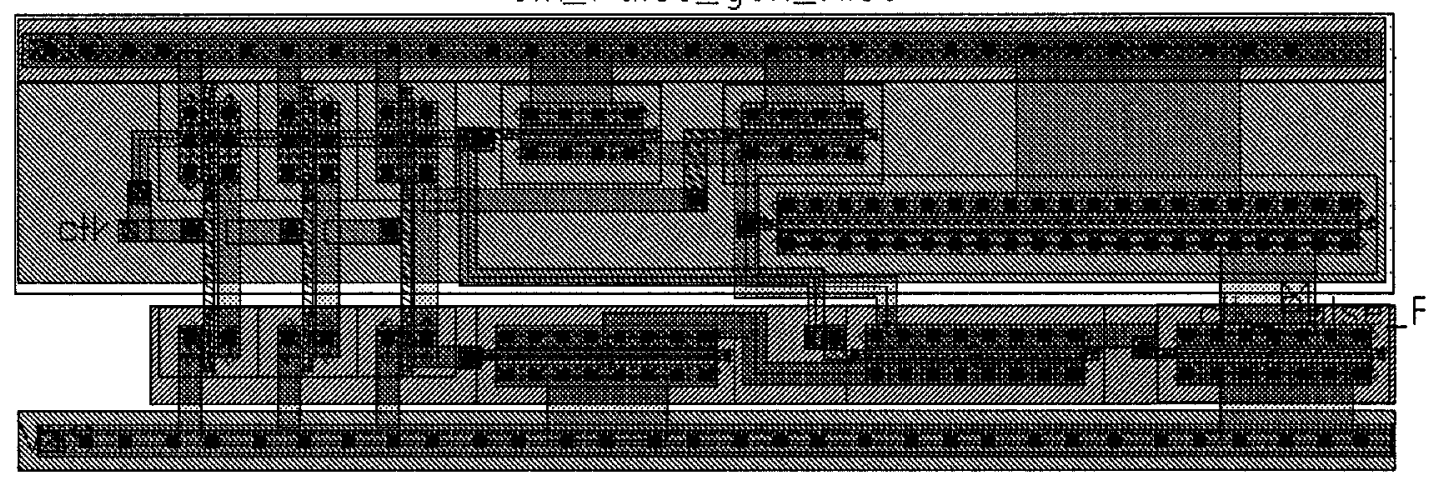

Figure C-8 Pulse generator for D flip flop (rise edge) 


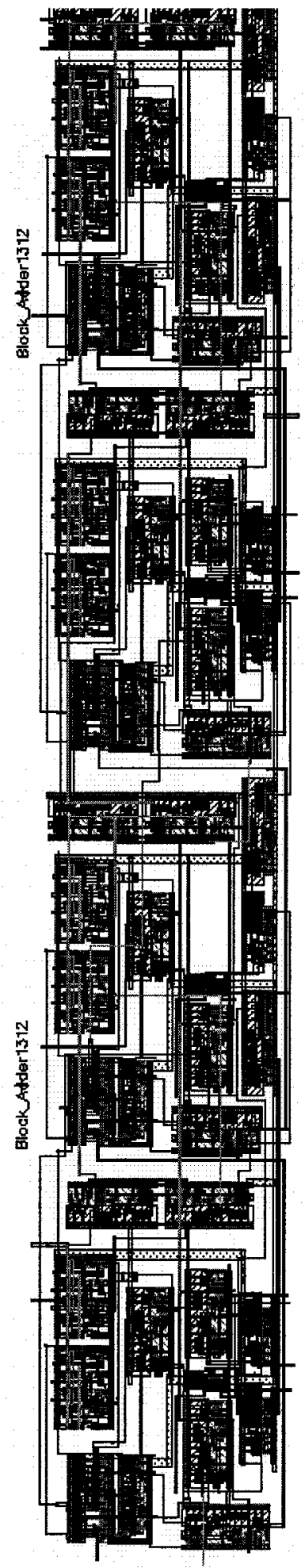

Figure C-9 Standard 8-bit modular multiplier 


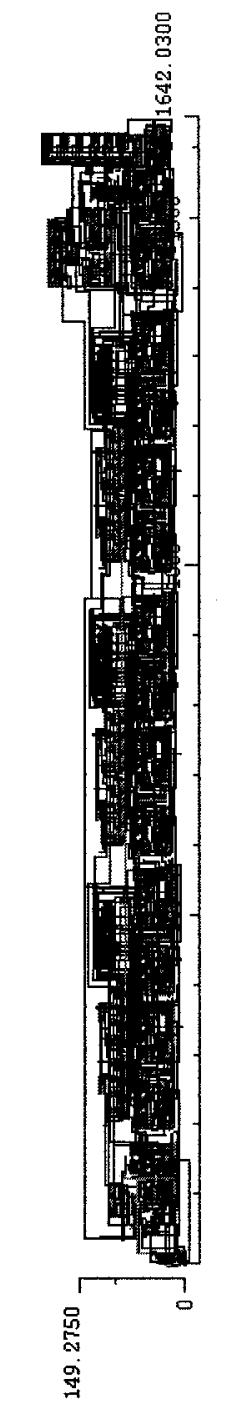

Figure C-10 32-bit modular multiplier 